\title{
Synthesis of Imidazole-Based [30]Heptaphyrin and Stable Figure-Eight [60]Tetradecaphyrins via [5+2] Condensations in One Pot
}

Chengming Li, Zhenmei Huang, Yanmei Hu, Wenbo Liang, Rongchuan Su, Mao Chen, Linsen Zhou, Di Wu, Ge Gao, * and Jingsong You*

†Key laboratory of Green Chemistry and Technology of Ministry of Education, College of Chemistry, Sichuan University, 29 Wangjiang Road, Chengdu 610064, P. R. China;

*Department of Cardiology, Laboratory of Heart Valve Disease, West China Hospital, Sichuan University, 37 Guoxue Road, Chengdu 610041, PR China

${ }^{\S}$ State Key Laboratory of Rare Earth Resource Utilization, Institute of Materials, Chinese Academy of Engineering Physics, Jiangyou 621908, PR China.

E-mail: gg2b@scu.edu.cn; jsyou@scu.edu.cn 


\section{Table of Contents}

1. Experimental section

1.1 General remarks. S2

1.2 Synthetic procedures and characterization data S2

2. Single crystal X-ray crystallographic data................................ 8

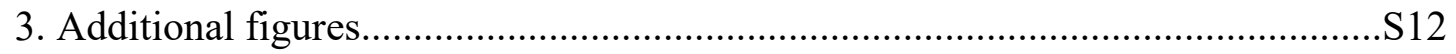

4. Schematic illustration of cyclization patterns.............................S21

5. Theoretical calculations................................................ 23

6. Copies of ${ }^{1} \mathrm{H},{ }^{13} \mathrm{C}$ NMR and Mass spectra of compounds..................... 29

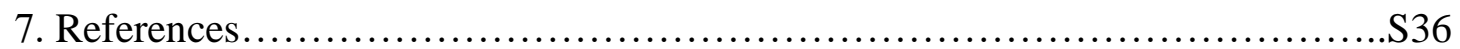

8. Appendix: Cartesian coordinates of the optimized structures..................S37 


\section{Experimental section}

\subsection{General remarks}

NMR spectra were recorded on a Varian Inova 400, or a Bruker AV II-400 spectrometer. The ${ }^{1} \mathrm{H}$ NMR (400 MHz) chemical shifts were recorded relative to $\mathrm{CDCl}_{3}$, benzene- $d_{6}$, toluene- $d_{8}$, acetone- $d_{6}$ or DMSO- $d_{6}$ as the internal reference $\left(\mathrm{CDCl}_{3}: \delta_{\mathrm{H}}=7.26 \mathrm{ppm}\right.$; toluene- $d_{8}: \delta_{\mathrm{H}}=2.08,6.97,7.01$ and 7.09 ppm; DMSO- $d_{6}: \delta_{\mathrm{H}}$ $=2.50 \mathrm{ppm} ; 1$ 1,1,2,2-tetrachloroethane- $\left.d_{2}: 6.00 \mathrm{ppm}\right)$. The ${ }^{13} \mathrm{C}$ NMR (100 MHz) chemical shifts were given using $\mathrm{CDCl}_{3}$ or DMSO- $d_{6}$ as the internal standard $\left(\mathrm{CDCl}_{3}\right.$ : $\left.\delta_{\mathrm{C}}=77.16 \mathrm{ppm} ; \mathrm{DMSO}-d_{6}: \delta_{\mathrm{C}}=39.52 \mathrm{ppm}\right)$. The solubility of partial product proved too low in common organic solvents to allow the ${ }^{13} \mathrm{C}$ NMR spectrum to be recorded. High-resolution mass spectra (HRMS) were obtained with a Shimadzu LCMS-IT-TOF (ESI). MALDI-TOF mass spectra were obtained with a Bruker Autoflex III smartbeam MALDI TOF spectrometer with matrix of -cyano-4-hydroxycinnamic acid (CCA). HPLC analyses were performed on Agilent 1260 Infinity II. Chiralpak IA column was purchased from Daicel ${ }^{\circledR}$. X-Ray single-crystal diffraction data were collected on a Bruker D8 VENTURE I $\mu$ S Diamond Duo with PHOTON III detector. UV/Vis spectra were measured on a HITACHI U-2910.

\subsection{Synthetic procedures and characterization data}

\section{Compounds 1 and 2}

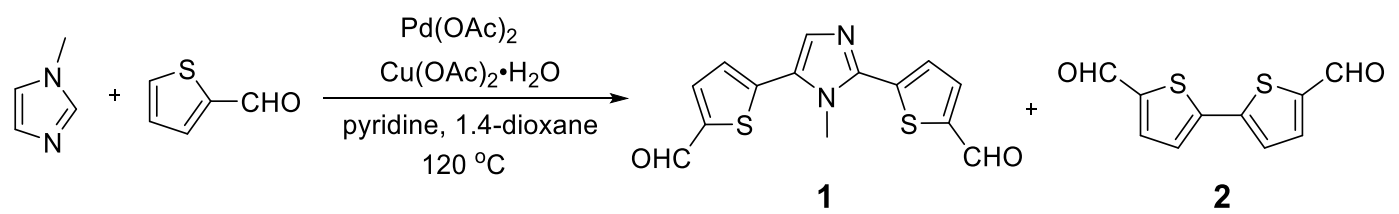

Compounds 1 and $\mathbf{2}$ were prepared in one pot by a modified procedure from our previous work. ${ }^{1}$ A schlenk tube with a magnetic stir bar was charged with $\mathrm{Pd}(\mathrm{OAc})_{2}$ (5.6 mg, $0.025 \mathrm{mmol}), \mathrm{Cu}(\mathrm{OAc})_{2} \cdot \mathrm{H}_{2} \mathrm{O}$ (300 mg, $\left.1.5 \mathrm{mmol}\right)$, 1-methylimidazole (41.1 $\mathrm{mg}, 0.5 \mathrm{mmol})$, 2-thenaldehyde (168.2 mg, $1.5 \mathrm{mmol})$, pyridine (39.6 mg, $0.5 \mathrm{mmol}$ ), and 1,4-dioxane $(0.6 \mathrm{~mL})$ under $\mathrm{N}_{2}$ atmosphere. The resulting solution was stirred at 
room temperature for $10 \mathrm{~min}$ and then heated at $120^{\circ} \mathrm{C}$ in an oil bath for $48 \mathrm{~h}$. After being cooling down, the reaction mixture was filtered through a Celite pad, and then washed with $30 \mathrm{~mL}$ of $\mathrm{CH}_{2} \mathrm{Cl}_{2}$. The solvent of the filtrate was removed under reduced pressure. The purification was performed by column chromatography on silica gel $\left(\mathrm{CH}_{2} \mathrm{Cl}_{2} /\right.$ ethyl acetate $=6: 1$ to $\left.4: 1, \mathrm{v} / \mathrm{v}\right)$ to provide 1 as a brownish yellow solid $(38.5$ $\mathrm{mg}, 25 \%$ yield) and 5,5'-diformyl-2,2'-bithiophene $\mathbf{2}$ as a yellow solid (28.8 $\mathrm{mg}, 17 \%$ yield). Compound 1: ${ }^{1} \mathrm{H}$ NMR (400 MHz, $\left.\mathrm{CDCl}_{3}\right): \delta(\mathrm{ppm}) 9.97(\mathrm{~s}, 1 \mathrm{H}), 9.93(\mathrm{~s}, 1 \mathrm{H})$, 7.81-7.79 (m, 2H), 7.57 (d, $J=4.0 \mathrm{~Hz}, 1 \mathrm{H}), 7.45$ (s, 1H), 7.28 (d, $J=4.0 \mathrm{~Hz}, 1 \mathrm{H})$, 3.98 (s, 3H). Compound 2: ${ }^{1} \mathrm{H}$ NMR (400 MHz, $\left.\mathrm{CDCl}_{3}\right): \delta(\mathrm{ppm}) 9.92$ (s, 2H), 7.73 (d, $J=4.0 \mathrm{~Hz}, 2 \mathrm{H}), 7.43(\mathrm{~d}, J=4.0 \mathrm{~Hz}, 2 \mathrm{H})$.

\section{Compound 3}

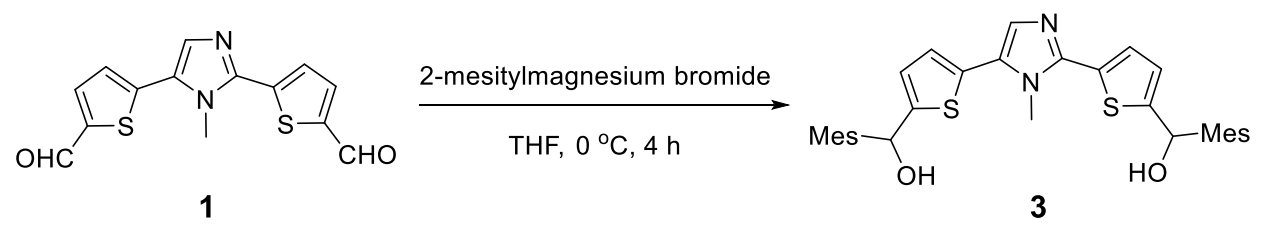

Freshly prepared 2-mesitylmagnesium bromide $(5.1 \mathrm{~mL}, 1.0 \mathrm{M}$ solution in THF, 5.1 mmol) was added to a well-stirred solution of 1 (500 mg, $1.7 \mathrm{mmol})$ in THF (20 mL) at $0{ }^{\circ} \mathrm{C}$ under $\mathrm{N}_{2}$ atmosphere. The mixture was stirred at the same temperature for $4 \mathrm{~h}$ and then allowed to raise to room temperature. The reaction mixture was then poured into a saturated aqueous solution of $\mathrm{NH}_{4} \mathrm{Cl}$ and extracted with ethyl acetate. The organic layer was dried over anhydrous $\mathrm{Na}_{2} \mathrm{SO}_{4}$ and concentrated by rotary evaporator. The residue was purified by column chromatography on silica gel $\left(\mathrm{CH}_{2} \mathrm{Cl}_{2} /\right.$ ethyl acetate $=3: 1, \mathrm{v} / \mathrm{v})$ to provide 3 as a faint yellow solid in $68 \%$ yield $(630 \mathrm{mg}) .{ }^{1} \mathrm{H}$ NMR (400 MHz, DMSO-d6): $\delta(\mathrm{ppm}) 7.26$ (d, $J=4.0 \mathrm{~Hz}, 1 \mathrm{H}), 7.06$ (s, 1H), 7.03 (d, $J=3.6 \mathrm{~Hz}, 1 \mathrm{H}), 6.83(\mathrm{~s}, 4 \mathrm{H}), 6.54-6.52(\mathrm{~m}, 2 \mathrm{H}), 6.26-6.25(\mathrm{~m}, 2 \mathrm{H}), 6.18-6.16(\mathrm{~m}$, 2H), $3.75(\mathrm{~s}, 3 \mathrm{H}), 2.27(\mathrm{~s}, 12 \mathrm{H}), 2.22(\mathrm{~s}, 6 \mathrm{H}) .{ }^{13} \mathrm{C}$ NMR (100 MHz, DMSO-d6): $\delta$ (ppm) 151.1, 150.4, 143.2, 136.9, 136.8, 136.2, 131.2, 129.5, 128.5, 128.3, 127.7, 126.2, 125.4, 123.3, 123.1, 67.2, 33.2, 20.5, 20.20, 20.17. HRMS (ESI) m/z: $[\mathrm{M}+\mathrm{H}]^{+}$ Calcd for $\mathrm{C}_{32} \mathrm{H}_{35} \mathrm{~N}_{2} \mathrm{O}_{2} \mathrm{~S}_{2}$ 543.2134; Found 543.2142.

\section{Compound $4^{2}$}




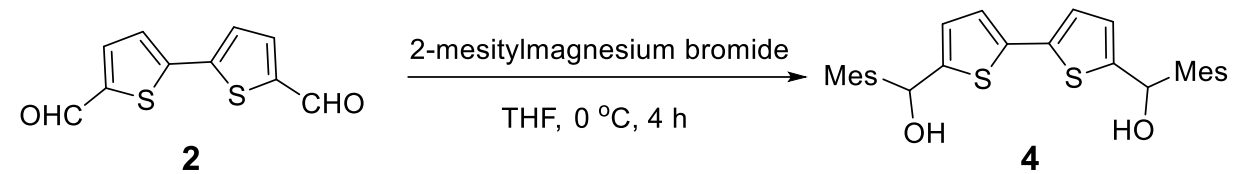

Freshly prepared 2-mesitylmagnesium bromide $(3.6 \mathrm{~mL}, 1.0 \mathrm{M}$ solution in THF, 3.6 mmol) was added to a well-stirred solution of 2 (267 $\mathrm{mg}, 1.2 \mathrm{mmol})$ in THF (16 mL) at $0{ }^{\circ} \mathrm{C}$ under $\mathrm{N}_{2}$ atmosphere. The mixture was stirred at same temperature for $4 \mathrm{~h}$ and allowed to room temperature. The reaction mixture was then poured into an aqueous saturated solution of $\mathrm{NH}_{4} \mathrm{Cl}$ and extracted with ethyl acetate. The organic layer was dried over anhydrous $\mathrm{Na}_{2} \mathrm{SO}_{4}$ and concentrated by rotary evaporator. The residue was purified by column chromatography on silica gel $\left(\mathrm{CH}_{2} \mathrm{Cl}_{2} /\right.$ ethyl acetate $\left.=10: 1, \mathrm{v} / \mathrm{v}\right)$ to provide 4 as a white solid in $81 \%$ yield $(450 \mathrm{mg}) .{ }^{1} \mathrm{H}$ NMR (400 MHz, DMSO- $\left.d_{6}\right): \delta$ (ppm) $6.96(\mathrm{~d}, J=3.6 \mathrm{~Hz}, 2 \mathrm{H}), 6.81(\mathrm{br}, 4 \mathrm{H}), 6.40-6.38(\mathrm{~m}, 2 \mathrm{H}), 6.19$ (d, $J=4.0 \mathrm{~Hz}$, $2 \mathrm{H}), 6.11(\mathrm{~d}, J=4.4 \mathrm{~Hz}, 2 \mathrm{H}), 2.24(\mathrm{~s}, 12 \mathrm{H}), 2.21(\mathrm{~s}, 6 \mathrm{H})$.

\section{Compound 5}
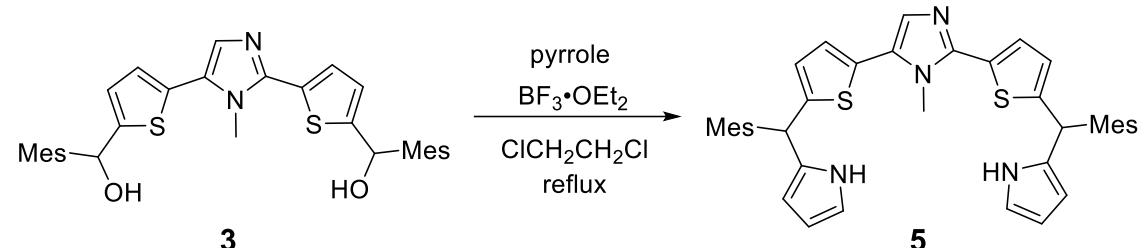

According to a previous procedure ${ }^{3}$, a mixture of compound 3 (600 mg, $\left.1.1 \mathrm{mmol}\right)$ and pyrrole $(3.5 \mathrm{~mL})$ in 1,2-dichloroethane $(15 \mathrm{~mL})$ was kept in $\mathrm{N}_{2}$ atmosphere for 10 min. Then $\mathrm{BF}_{3} \cdot \mathrm{Et}_{2} \mathrm{O}(0.5 \mathrm{~mL})$ was added and the resulting mixture was stirred under reflux in an oil bath overnight. The solution was cooled to room temperature and quenched by addition of triethylamine $(0.8 \mathrm{~mL})$. The mixture was extracted with $\mathrm{CH}_{2} \mathrm{Cl}_{2}$, dried over anhydrous $\mathrm{Na}_{2} \mathrm{SO}_{4}$ and concentrated by rotary evaporator. The residue was purified by column chromatography on silica gel $\left(\mathrm{CH}_{2} \mathrm{Cl}_{2} /\right.$ ethyl acetate $=$ 10:1, v/v) to provide 5 as a yellow solid in $65 \%$ yield $(460 \mathrm{mg}) .{ }^{1} \mathrm{H}$ NMR (400 MHz, $\left.\mathrm{CDCl}_{3}\right): \delta(\mathrm{ppm}) 7.91(\mathrm{br}, 2 \mathrm{H}), 7.17(\mathrm{~d}, J=3.6 \mathrm{~Hz}, 1 \mathrm{H}), 7.11(\mathrm{~s}, 1 \mathrm{H}), 6.92(\mathrm{~d}, J=3.6$ $\mathrm{Hz}, 1 \mathrm{H}), 6.89-6.86$ (m, 6H), 6.70-6.66 (m, 2H), 6.20-6.17 (m, 2H), 6.14-6.11 (m, 2H), $6.08(\mathrm{~s}, 2 \mathrm{H}), 3.78(\mathrm{~s}, 3 \mathrm{H}), 2.29(\mathrm{~s}, 3 \mathrm{H}), 2.27(\mathrm{~s}, 3 \mathrm{H}), 2.16(\mathrm{~s}, 6 \mathrm{H}), 2.14(\mathrm{~s}, 6 \mathrm{H}),{ }^{13} \mathrm{C}$ NMR $\left(\mathrm{CDCl}_{3}, 100 \mathrm{MHz}\right): \delta(\mathrm{ppm}) 147.3,146.8,143.9,137.5,137.1,137.0,135.41$, 
$135.40,131.84,131.81,131.7,130.6,130.5,129.4,128.8,128.5,126.22,126.20$, $126.15,125.4$, 116.7, 116.6, 108.7, 108.6, 107.1, 40.65, 40.57, 33.4, 20.99, 20.97, 20.9. HRMS (ESI) m/z: $[\mathrm{M}+\mathrm{H}]^{+}$Calcd for $\mathrm{C}_{40} \mathrm{H}_{41} \mathrm{~N}_{4} \mathrm{~S}_{2}$ 641.2767; Found 641.2771.

\section{Compound $6^{4}$}<smiles>CC(O)c1ccc(-c2ccc(C(C)O)s2)s1</smiles>

4

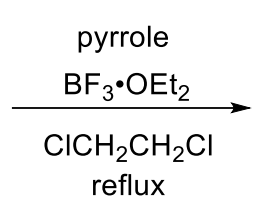

reflux

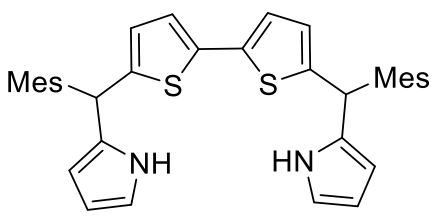

6

A mixture of compound $4(370 \mathrm{mg}, 0.8 \mathrm{mmol})$ and pyrrole $(2.5 \mathrm{~mL})$ in 1,2-dichloroethane $(10 \mathrm{~mL})$ was kept in $\mathrm{N}_{2}$ atmosphere for 10 min. Then $\mathrm{BF}_{3} \cdot \mathrm{Et}_{2} \mathrm{O}$ $(0.37 \mathrm{~mL})$ was added and the resulting mixture was stirred under reflux in an oil bath overnight. The solution was cooled to room temperature and quenched by addition of triethylamine $(0.6 \mathrm{~mL})$. The mixture was extracted with $\mathrm{CH}_{2} \mathrm{Cl}_{2}$, dried over anhydrous $\mathrm{Na}_{2} \mathrm{SO}_{4}$ and concentrated by rotary evaporator. The residue was purified by column chromatography on silica gel $\left(\mathrm{CH}_{2} \mathrm{Cl}_{2} /\right.$ ethyl acetate $\left.=30: 1, \mathrm{v} / \mathrm{v}\right)$ to provide $\mathbf{6}$ as a white solid in $60 \%$ yield $\left(270 \mathrm{mg}\right.$ ). ${ }^{1} \mathrm{H}$ NMR (400 MHz, $\left.\mathrm{CDCl}_{3}\right): \delta(\mathrm{ppm}) 7.87$ (br, 2H), $6.91(\mathrm{~d}, J=3.6 \mathrm{~Hz}, 2 \mathrm{H}), 6.87(\mathrm{br}, 4 \mathrm{H}), 6.72-6.71(\mathrm{~m}, 2 \mathrm{H}), 6.68-6.66(\mathrm{~m}, 2 \mathrm{H})$, 6.19-6.17 (m, 2H), 6.10-6.08 (m, 2H), $6.02(\mathrm{~s}, 2 \mathrm{H}), 2.28(\mathrm{~s}, 6 \mathrm{H}), 2.13(\mathrm{~s}, 12 \mathrm{H})$.

\section{Compounds 7-9}
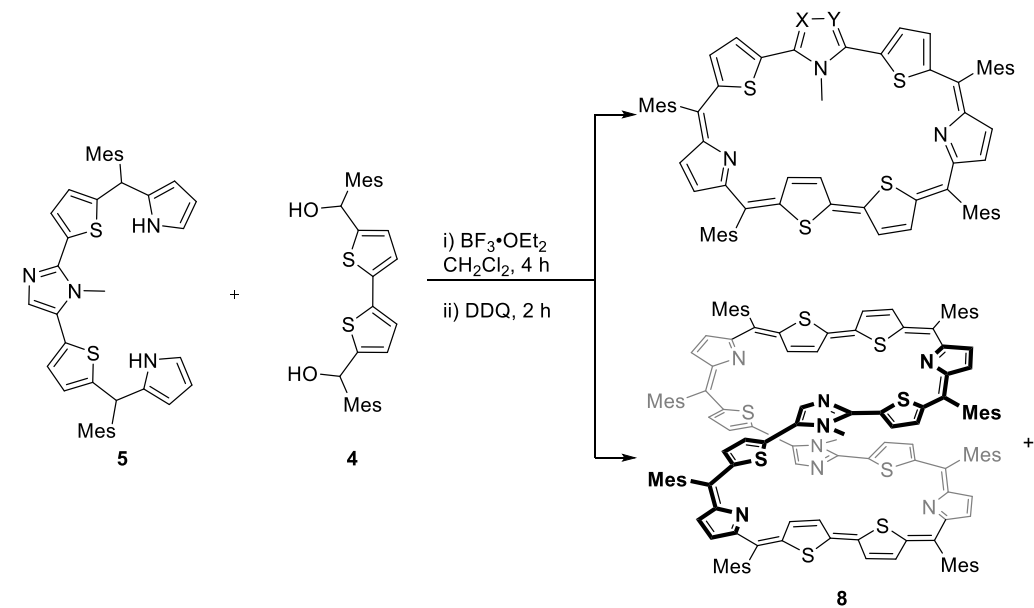

$7 \mathbf{a}: \mathrm{X}=\mathrm{CH}, \mathrm{Y}=\mathrm{N} ; \mathbf{7 b}: \mathrm{X}=\mathrm{N}, \mathrm{Y}=\mathrm{CH}$

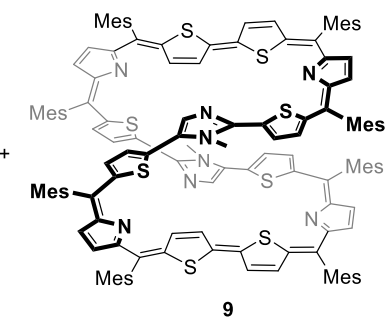

To a $100 \mathrm{~mL}$ dry $\mathrm{CH}_{2} \mathrm{Cl}_{2}$ solution, compound 5 (103 $\left.\mathrm{mg}, 0.16 \mathrm{mmol}\right)$ and 5,5'-bis-[(2,4,6-trimethylphenyl)hydroxymethyl]-2,2'-bithiophene diol 4 (96 mg, 0.2 mmol) were added under $\mathrm{N}_{2}$ atmosphere and stirred for 10 min. Then $\mathrm{BF}_{3} \cdot \mathrm{OEt}_{2}(20$ 
$\mu \mathrm{L}, 0.16 \mathrm{mmol}$ ) was added dropwise, after which the mixture was stirred at room temperature for $4 \mathrm{~h}$ under dark. 2,3- Dichloro-5,6-dicyano-1,4-benzoquinone (DDQ; $109 \mathrm{mg}, 0.48 \mathrm{mmol}$ ) was added and the vessel was opened to the air. The reaction mixture was further stirred for $2 \mathrm{~h}$. The solvent was removed on rotary evaporator under vacuum and the residue was firstly subjected to basic alumina column chromatographic. Then the crude compound was purified by neutralized alumina column chromatographic with $\mathrm{CH}_{2} \mathrm{Cl}_{2}$-ethyl acetate $(10: 1$ to $5: 1, \mathrm{v} / \mathrm{v})$ as eluent. The first dark band was collected to afford the desired product 8 as a black solid (13 mg, 8\% yield). The second dark band was collected to give the product 9 as a black solid (8 $\mathrm{mg}, 5 \%$ yield). The third deep red band was collected to afford product 7 as a black solid containing a mixture of two different conformers in a ratio of ca. 1:0.85 (35 $\mathrm{mg}$, $21 \%$ yield).

Compound 8: ${ }^{1} \mathrm{H}$ NMR (400 MHz, $\mathrm{CDCl}_{3}$, r.t.): $\delta$ (ppm) $9.82(\mathrm{~d}, J=5.6 \mathrm{~Hz}, 2 \mathrm{H})$, $9.40(\mathrm{~d}, J=5.6 \mathrm{~Hz}, 2 \mathrm{H}), 8.19(\mathrm{~s}, 2 \mathrm{H}), 7.19(\mathrm{~d}, J=4.4 \mathrm{~Hz}, 2 \mathrm{H}), 7.08(\mathrm{~s}, 2 \mathrm{H})$, 6.99-6.91 (m, 16H), 6.87 (s, 2H), 6.84 (d, $J=4.0 \mathrm{~Hz}, 2 \mathrm{H}), 6.49$ (br, 4H), 6.38 (d, $J=$ $4.8 \mathrm{~Hz}, 2 \mathrm{H}), 6.35(\mathrm{~d}, J=4.8 \mathrm{~Hz}, 2 \mathrm{H}), 6.05(\mathrm{~d}, J=4.8 \mathrm{~Hz}, 2 \mathrm{H}), 6.01(\mathrm{~d}, J=4.4 \mathrm{~Hz}$, 2H), $3.93(\mathrm{~s}, 6 \mathrm{H}), 2.45(\mathrm{~s}, 6 \mathrm{H}), 2.38(\mathrm{~s}, 6 \mathrm{H}), 2.37(\mathrm{~s}, 6 \mathrm{H}), 2.33(\mathrm{~s}, 6 \mathrm{H}), 2.26(\mathrm{~s}, 6 \mathrm{H})$, $2.22(\mathrm{~s}, 6 \mathrm{H}), 2.10(\mathrm{~s}, 6 \mathrm{H}), 2.05(\mathrm{~s}, 6 \mathrm{H}), 2.03(\mathrm{~s}, 6 \mathrm{H}), 2.02(\mathrm{~s}, 6 \mathrm{H}), 1.99(\mathrm{~s}, 6 \mathrm{H}), 1.92$ (s, 6H). COSY NMR (400 MHz, $\mathrm{CDCl}_{3}$, r.t.) (Figure S12) and NOESY NMR (400 MHz, $\mathrm{CDCl}_{3}$, r.t.) (Figure $\mathrm{S} 13$ ) were recorded for further structural assignment. MS (MALDI-TOF) m/z: [M] $]^{+}$Calcd for $\mathrm{C}_{136} \mathrm{H}_{120} \mathrm{~N}_{8} \mathrm{~S}_{8} 2120.7402$; Found: 2120.7446.

Compound 9: ${ }^{1} \mathrm{H}$ NMR (400 MHz, $\mathrm{CDCl}_{3}$, r.t.): $\delta$ (ppm) $9.86(\mathrm{~d}, J=2.8 \mathrm{~Hz}, 1 \mathrm{H})$, $9.63(\mathrm{~d}, J=3.2 \mathrm{~Hz}, 1 \mathrm{H}), 9.22$ (d, $J=2.4 \mathrm{~Hz}, 1 \mathrm{H}), 8.19$ (s, 1H), 8.08 (s, 1H), 7.93 (d, $J=5.6 \mathrm{~Hz}, 1 \mathrm{H}), 7.71(\mathrm{~d}, J=3.2 \mathrm{~Hz}, 1 \mathrm{H}), 7.00-6.85(\mathrm{~m}, 23 \mathrm{H}), 6.49-6.44(\mathrm{~m}, 2 \mathrm{H})$, 6.39-6.35 (m, 5H), $6.28(\mathrm{~d}, J=2.0 \mathrm{~Hz}, 1 \mathrm{H}), 6.07-6.05(\mathrm{~m}, 2 \mathrm{H}), 6.00(\mathrm{~d}, J=4.0 \mathrm{~Hz}$, 1H), $5.98(\mathrm{~d}, J=4.4 \mathrm{~Hz}, 1 \mathrm{H}), 3.93(\mathrm{~s}, 3 \mathrm{H}), 3.92$ (s, 3H), 2.48 (s, 3H), 2.46 (s, 3H), 2.36-2.34 (m, 18H), $2.29(\mathrm{~s}, 3 \mathrm{H}), 2.26(\mathrm{~s}, 3 \mathrm{H}), 2.22(\mathrm{~s}, 3 \mathrm{H}), 2.19(\mathrm{~s}, 3 \mathrm{H}), 2.14(\mathrm{~s}, 3 \mathrm{H})$, $2.11(\mathrm{~s}, 3 \mathrm{H}), 2.10(\mathrm{~s}, 3 \mathrm{H}), 2.06-2.04(\mathrm{~m}, 15 \mathrm{H}), 2.01(\mathrm{~s}, 3 \mathrm{H}), 1.97(\mathrm{~s}, 3 \mathrm{H}), 1.95(\mathrm{~s}, 3 \mathrm{H})$, 1.93 (s, 3H). COSY NMR (400 MHz, $\mathrm{CDCl}_{3}$, r.t.) (Figure S14) was recorded for 
structural assignment. MS (MALDI-TOF) m/z: [M] $]^{+}$Calcd for $\mathrm{C}_{136} \mathrm{H}_{120} \mathrm{~N}_{8} \mathrm{~S}_{8}$ 2120.7402; Found: 2120.7491.

Conformer 7a: ${ }^{1} \mathrm{H}$ NMR (400 MHz, $\mathrm{CDCl}_{3}$, r.t.): $\delta$ (ppm) 10.23 (d, $\left.J=4.8 \mathrm{~Hz}\right), 9.49$ (d, $J=4.4 \mathrm{~Hz}), 9.48$ (s), 9.17 (d, $J=5.2 \mathrm{~Hz}), 8.87$ (d, $J=4.4 \mathrm{~Hz}), 8.75-8.73$ (m, overlaps with conformer 7b), $8.57(\mathrm{~d}, J=5.2 \mathrm{~Hz}), 7.90-7.88(\mathrm{t}, J=4.0 \mathrm{~Hz}), 7.64-7.62$ (m), -1.15 (s). Conformer 7b: ${ }^{1} \mathrm{H}$ NMR (400 MHz, $\mathrm{CDCl}_{3}$, r.t.): $\delta$ (ppm) 9.90 (d, $J=$ $4.4 \mathrm{~Hz}), 9.58$ (d, $J=4.4 \mathrm{~Hz}), 9.40$ (s), 9.11 (d, $J=5.6 \mathrm{~Hz}), 8.75-8.73$ (m, overlaps with conformer 7a), $8.52(\mathrm{~d}, J=5.2 \mathrm{~Hz}), 7.86-7.84(\mathrm{t}, J=4.8 \mathrm{~Hz}), 7.60-7.58(\mathrm{~m})$, -0.98 (s). Signals observed in the region of 1.14-1.35 ppm relate to one inverted thiophene protons. Resonances in the region of 7.32-7.23 ppm can be assigned to the aryl protons of 2-mesitylene ring. The resonances in the alkyl region of 2.62-2.07 ppm belong to the methyl protons of 2-mesitylene ring. HRMS (ESI) m/z: $[\mathrm{M}+\mathrm{H}]^{+} \mathrm{Calcd}$ for $\mathrm{C}_{68} \mathrm{H}_{61} \mathrm{~N}_{4} \mathrm{~S}_{4}$ 1061.3774; Found 1061.3773.

The solubility of compounds $\mathbf{7 - 9}$ is too low in common organic solvents to allow the ${ }^{13} \mathrm{C}$ NMR spectrum to be recorded. 


\section{Single crystal $X$-ray crystallographic data}

Single crystals for $\mathbf{8}$ and $\mathbf{9}$ were grown by the slow diffusion of $n$-hexane into a solution of a compound in dichloromethane, respectively. The X-ray crystallographic structures are ORTEP representation with 50\% probability thermal ellipsoids. All hydrogen atoms are omitted for clarity.

Table S1. Crystal data and structure refinement for 8

\begin{tabular}{|c|c|}
\hline Identification code & CCDC 2021732 \\
\hline Empirical formula & $\mathrm{C}_{136} \mathrm{H}_{120} \mathrm{~N}_{8} \mathrm{~S}_{8}$ \\
\hline Formula weight & 2122.87 \\
\hline Temperature/K & 187.54 \\
\hline Crystal system & monoclinic \\
\hline Space group & $\mathrm{P} 2{ }_{1} / \mathrm{c}$ \\
\hline $\mathrm{a} / \AA ̊$ & $18.7282(14)$ \\
\hline $\mathrm{b} / \AA$ & $38.747(3)$ \\
\hline$c / \AA$ & $19.952(2)$ \\
\hline$\alpha /^{\circ}$ & 90 \\
\hline$\beta /{ }^{\circ}$ & $92.773(4)$ \\
\hline$\gamma /{ }^{\circ}$ & 90 \\
\hline Volume $/ \AA^{3}$ & $14462(2)$ \\
\hline $\mathrm{Z}$ & 4 \\
\hline$\rho_{\text {calc }} \mathrm{g} / \mathrm{cm}^{3}$ & 0.975 \\
\hline$\mu / \mathrm{mm}^{-1}$ & 0.970 \\
\hline $\mathrm{F}(000)$ & 4480.0 \\
\hline Crystal size $/ \mathrm{mm}^{3}$ & $0.2 \times 0.1 \times 0.04$ \\
\hline Radiation & $\operatorname{GaK} \alpha(\lambda=1.34139)$ \\
\hline \multicolumn{2}{|c|}{$2 \Theta$ range for data collection/ $/{ }^{\circ} 5.846$ to 101.034} \\
\hline Index ranges & $-21 \leq \mathrm{h} \leq 21,-34 \leq \mathrm{k} \leq 44,-22 \leq 1 \leq 18$ \\
\hline Reflections collected & 65193 \\
\hline Independent reflections & $22773\left[R_{\text {int }}=0.0528, R_{\text {sigma }}=0.0633\right]$ \\
\hline Data/restraints/parameters & $22773 / 31 / 1453$ \\
\hline Goodness-of-fit on $\mathrm{F}^{2}$ & 1.037 \\
\hline Final $R$ indexes $[\mathrm{I}>=2 \sigma(\mathrm{I})]$ & $\mathrm{R}_{1}=0.0551, \mathrm{wR}_{2}=0.1570$ \\
\hline Final $R$ indexes [all data] & $\mathrm{R}_{1}=0.1104, \mathrm{wR}_{2}=0.1845$ \\
\hline Largest diff. peak/hole / e $\AA$ & $0.22 /-0.23$ \\
\hline
\end{tabular}




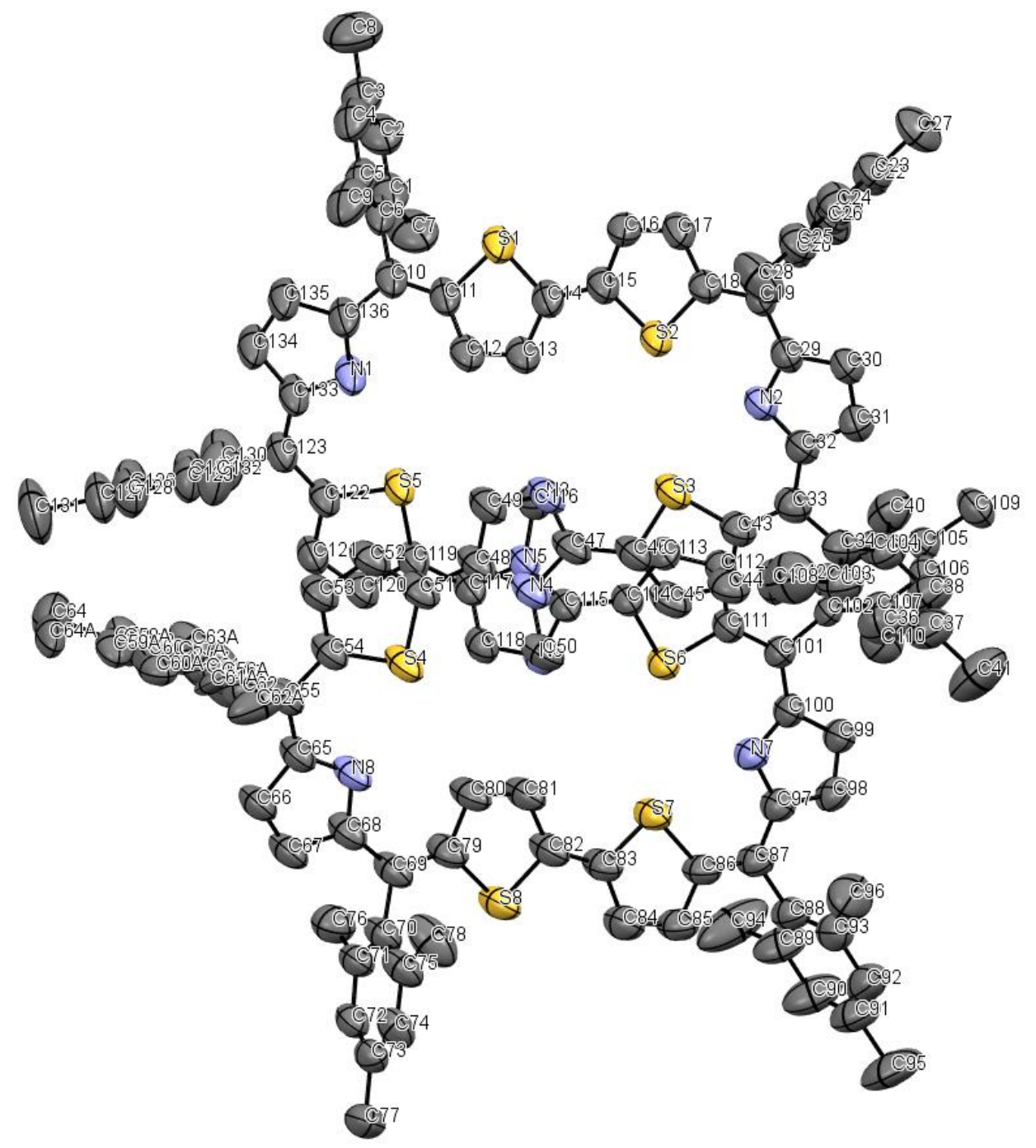


Table S2. Crystal data and structure refinement for 9

Identification code

CCDC 2021733

Empirical formula

$\mathrm{C}_{136} \mathrm{H}_{120} \mathrm{~N}_{8} \mathrm{~S}_{8}$

Formula weight

2122.87

Temperature/K

173.0

Crystal system

monoclinic

Space group

$\mathrm{P} 2{ }_{1} / \mathrm{c}$

$\mathrm{a} / \AA$

$19.0776(4)$

$\mathrm{b} / \AA$

$36.3369(9)$

$\mathrm{c} / \AA$

23.3619(8)

$\alpha /{ }^{\circ}$

90

$\beta /^{\circ}$

93.167(2)

$\gamma /{ }^{\circ}$

90

Volume $/ \AA^{3}$

16170.2(8)

$\mathrm{Z}$

$\rho_{\text {calc }} \mathrm{g} / \mathrm{cm}^{3}$

4

$\mu / \mathrm{mm}^{-1}$

0.872

1.322

$\mathrm{F}(000)$

4480.0

Crystal size/ $/ \mathrm{mm}^{3}$

$0.1 \times 0.1 \times 0.05$

Radiation

$\mathrm{CuK \alpha}(\lambda=1.54178)$

$2 \Theta$ range for data collection $/{ }^{\circ}$

6.166 to 137.414

Index ranges

$-23 \leq \mathrm{h} \leq 17,-43 \leq \mathrm{k} \leq 36,-28 \leq 1 \leq 27$

Reflections collected

85824

Independent reflections

$29336\left[R_{\text {int }}=0.0683, R_{\text {sigma }}=0.0551\right]$

Data/restraints/parameters

29336/3099/1395

Goodness-of-fit on $\mathrm{F}^{2}$

0.937

Final $\mathrm{R}$ indexes $[\mathrm{I}>=2 \sigma(\mathrm{I})]$

$\mathrm{R}_{1}=0.0658, \mathrm{wR}_{2}=0.1867$

Final $\mathrm{R}$ indexes [all data]

$\mathrm{R}_{1}=0.1123, \mathrm{wR}_{2}=0.2184$

Largest diff. peak/hole / e $\AA^{-3}$

$0.32 /-0.34$ 


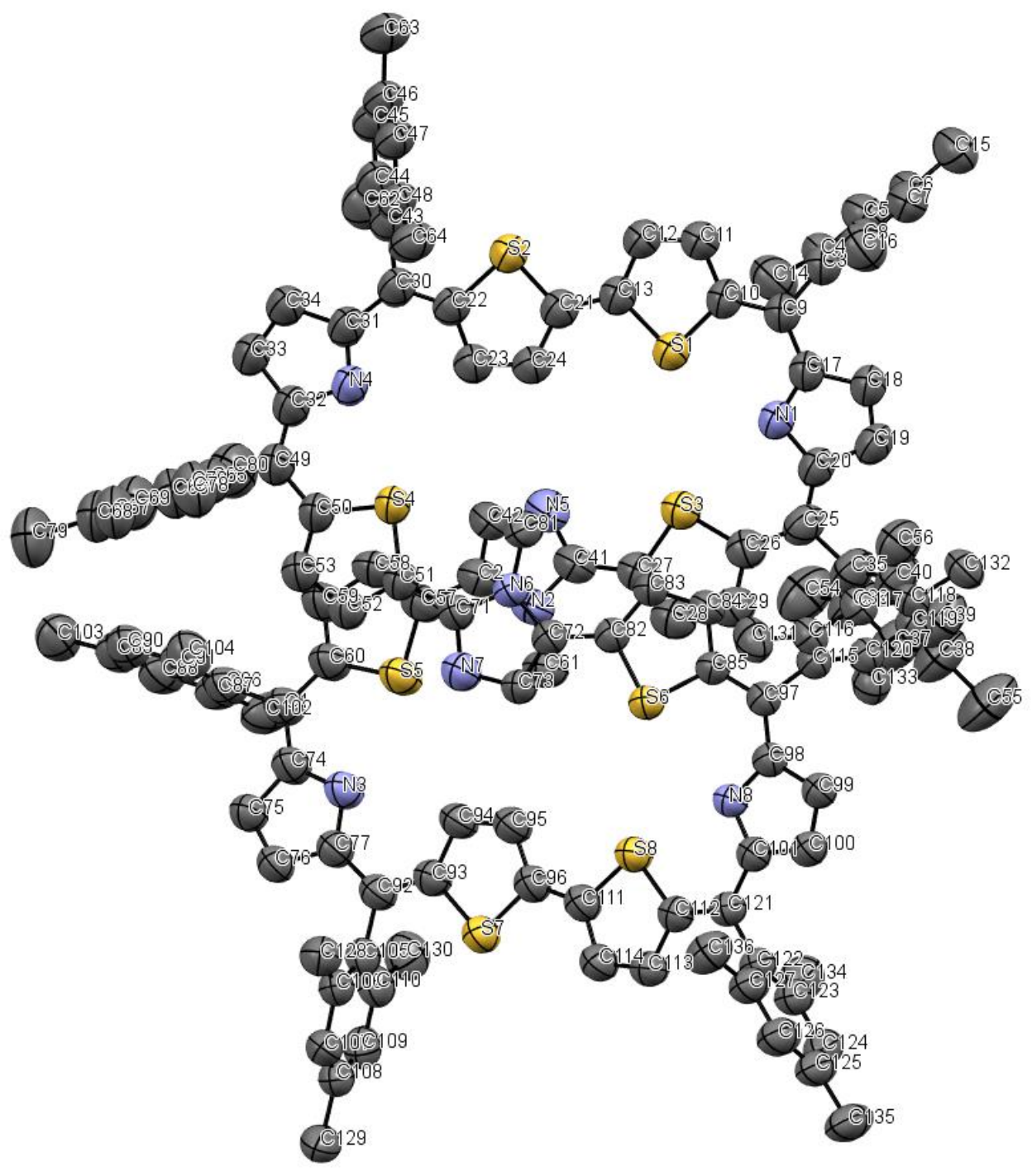




\section{Additional figures}

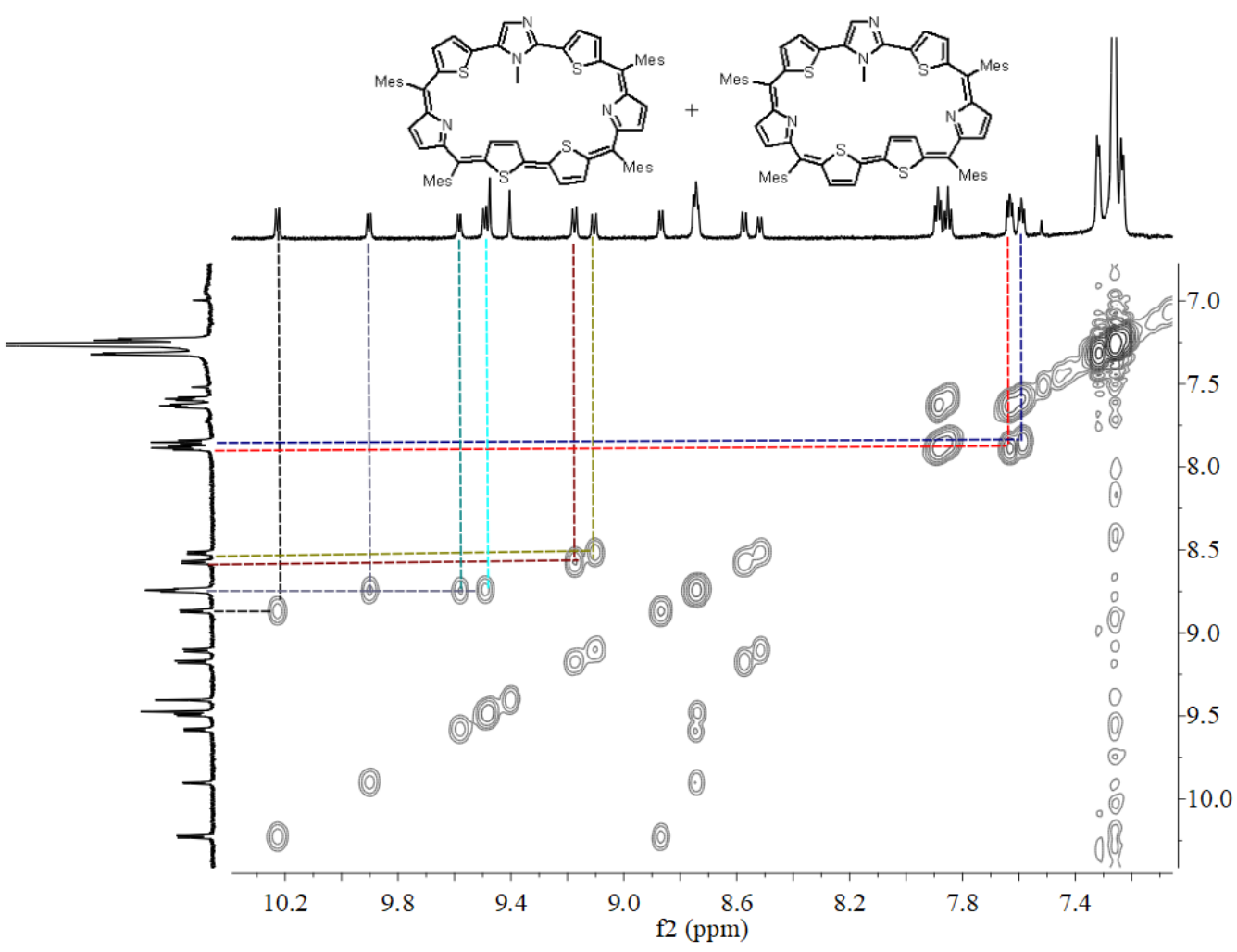

Figure S1. ${ }^{1} \mathrm{H}-{ }^{1} \mathrm{H}$ COSY correlation spectrum of 7 at $298 \mathrm{~K}\left(400 \mathrm{MHz}, \mathrm{CDCl}_{3}\right)$.

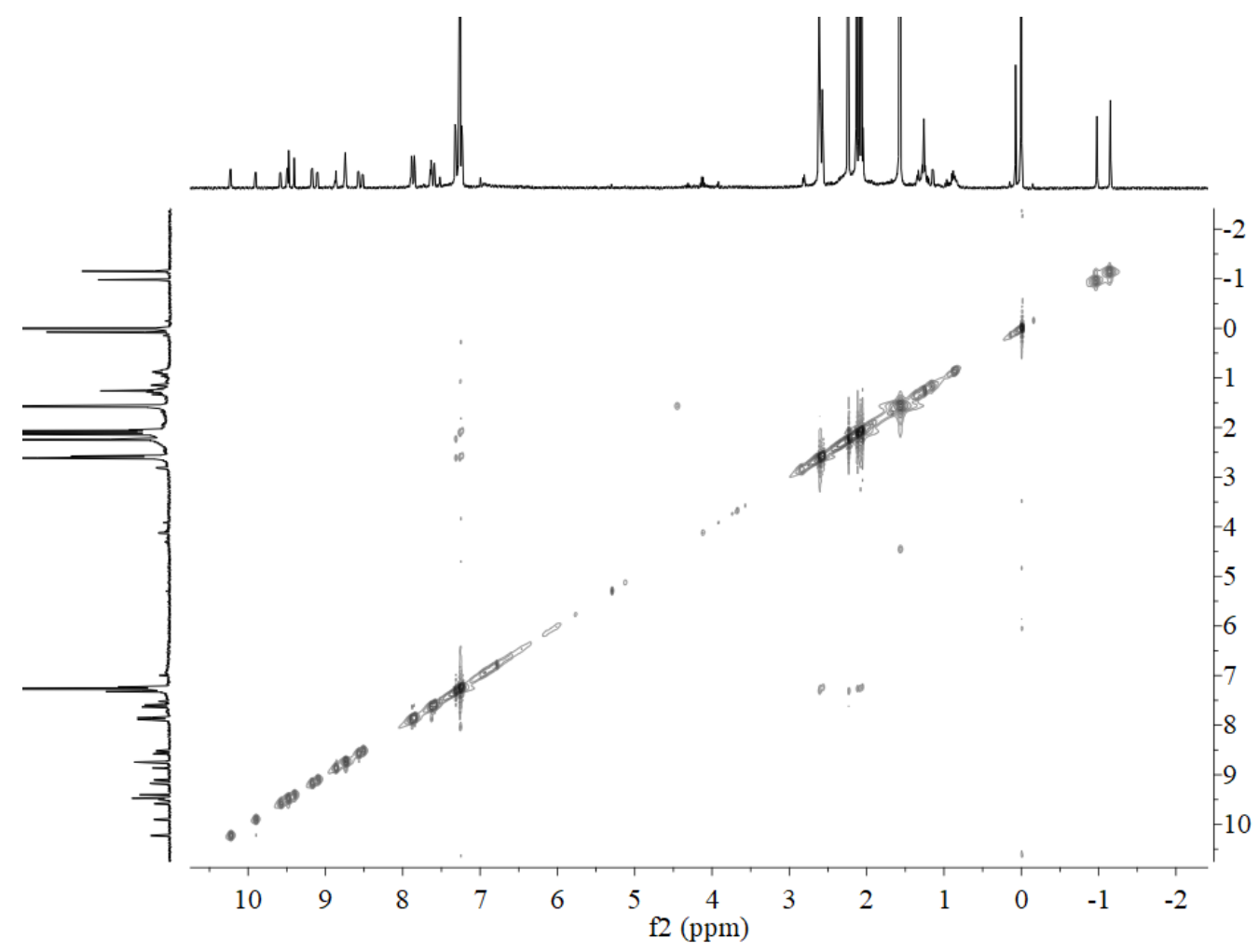

Figure S2. ${ }^{1} \mathrm{H}-{ }^{-1} \mathrm{H}$ NOESY correlation spectrum of 7 at $298 \mathrm{~K}\left(400 \mathrm{MHz}, \mathrm{CDCl}_{3}\right)$. 


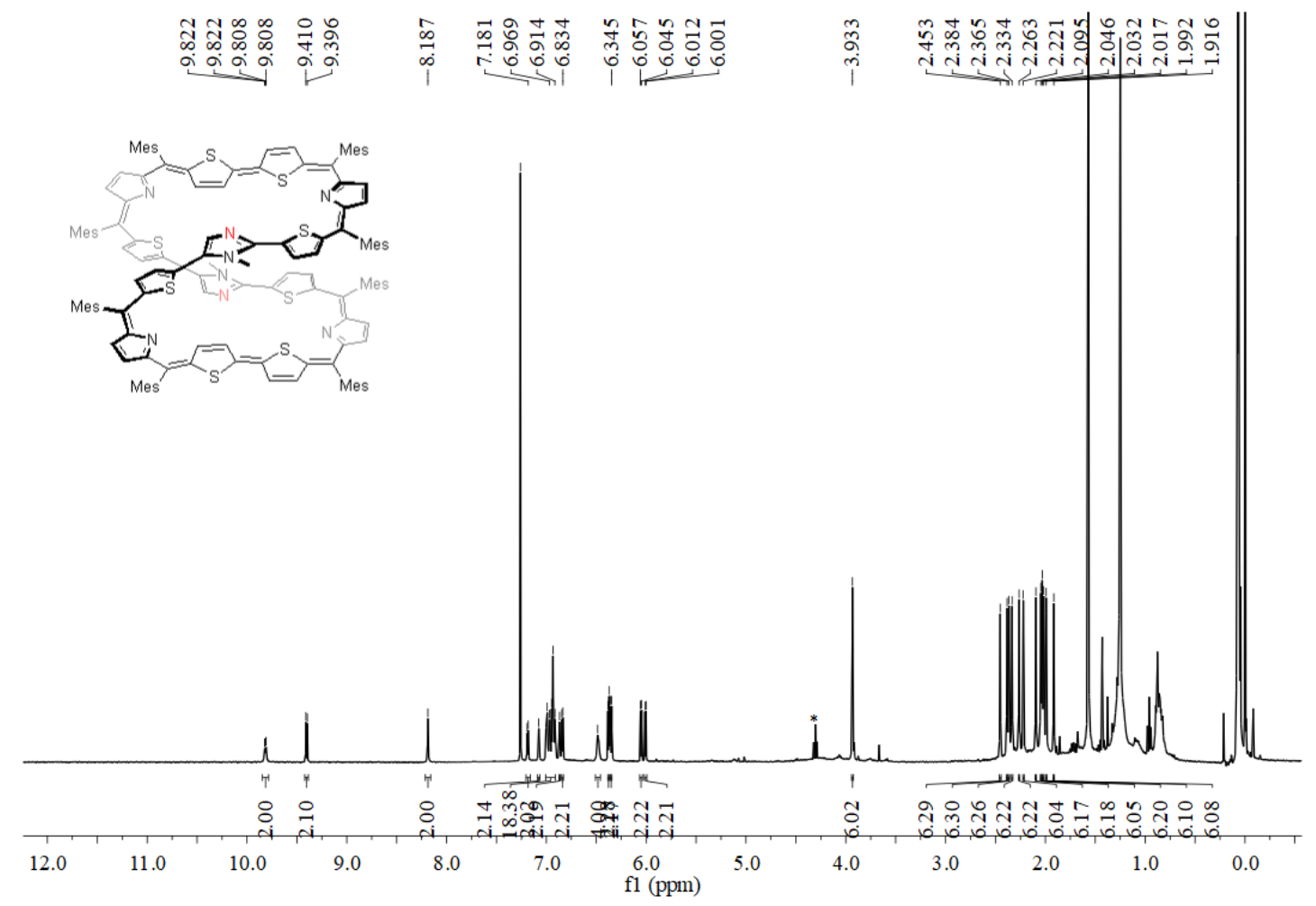

Figure S3. ${ }^{1} \mathrm{H}$ NMR spectrum of 8 at $298 \mathrm{~K}\left(400 \mathrm{MHz}, \mathrm{CDCl}_{3},{ }^{*}\right.$ residual solvent peaks).

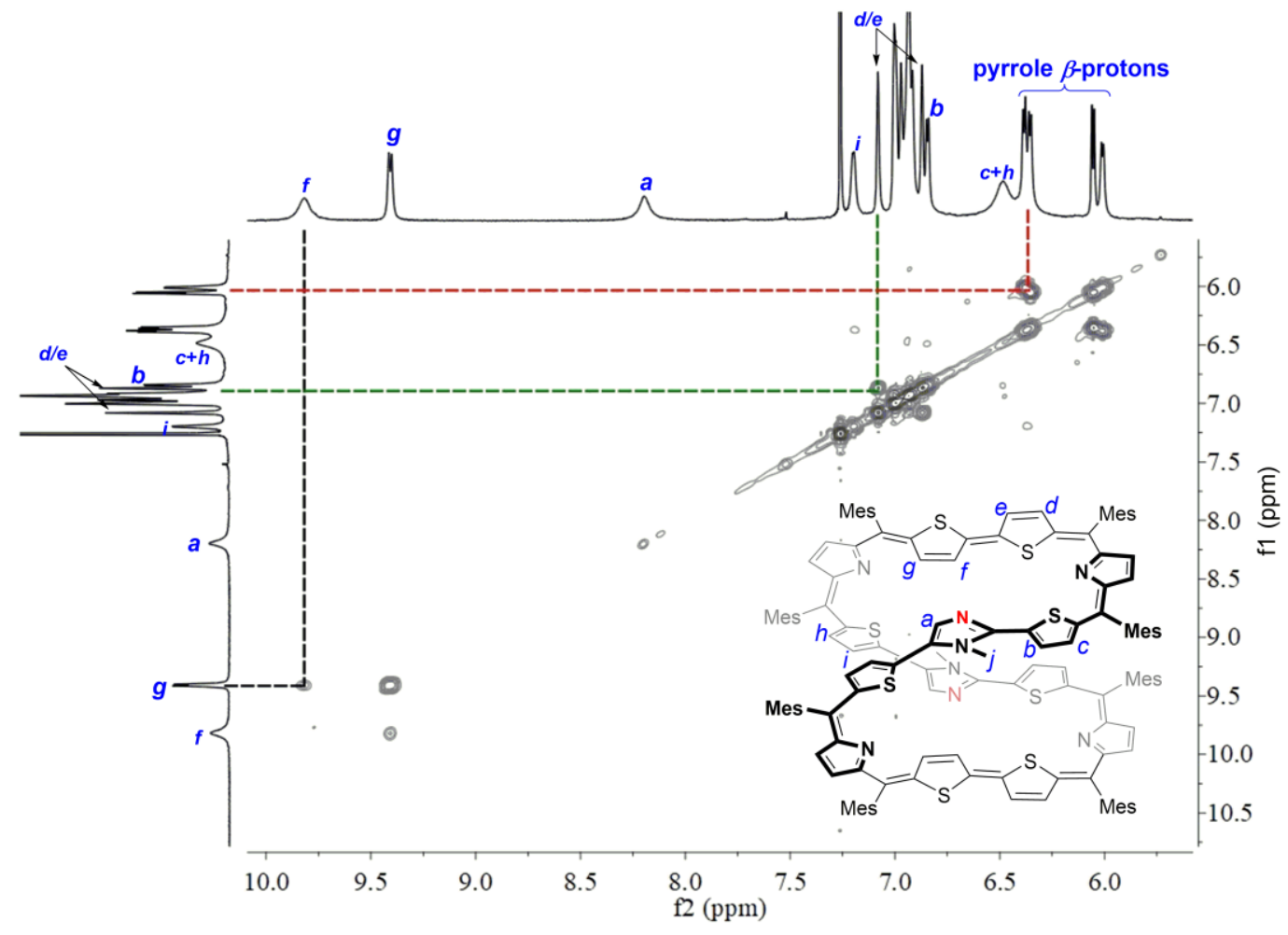

Figure S4. ${ }^{1} \mathrm{H}-{ }^{1} \mathrm{H}$ COSY correlation spectrum of 8 at $298 \mathrm{~K}\left(400 \mathrm{MHz}, \mathrm{CDCl}_{3}\right)$. 


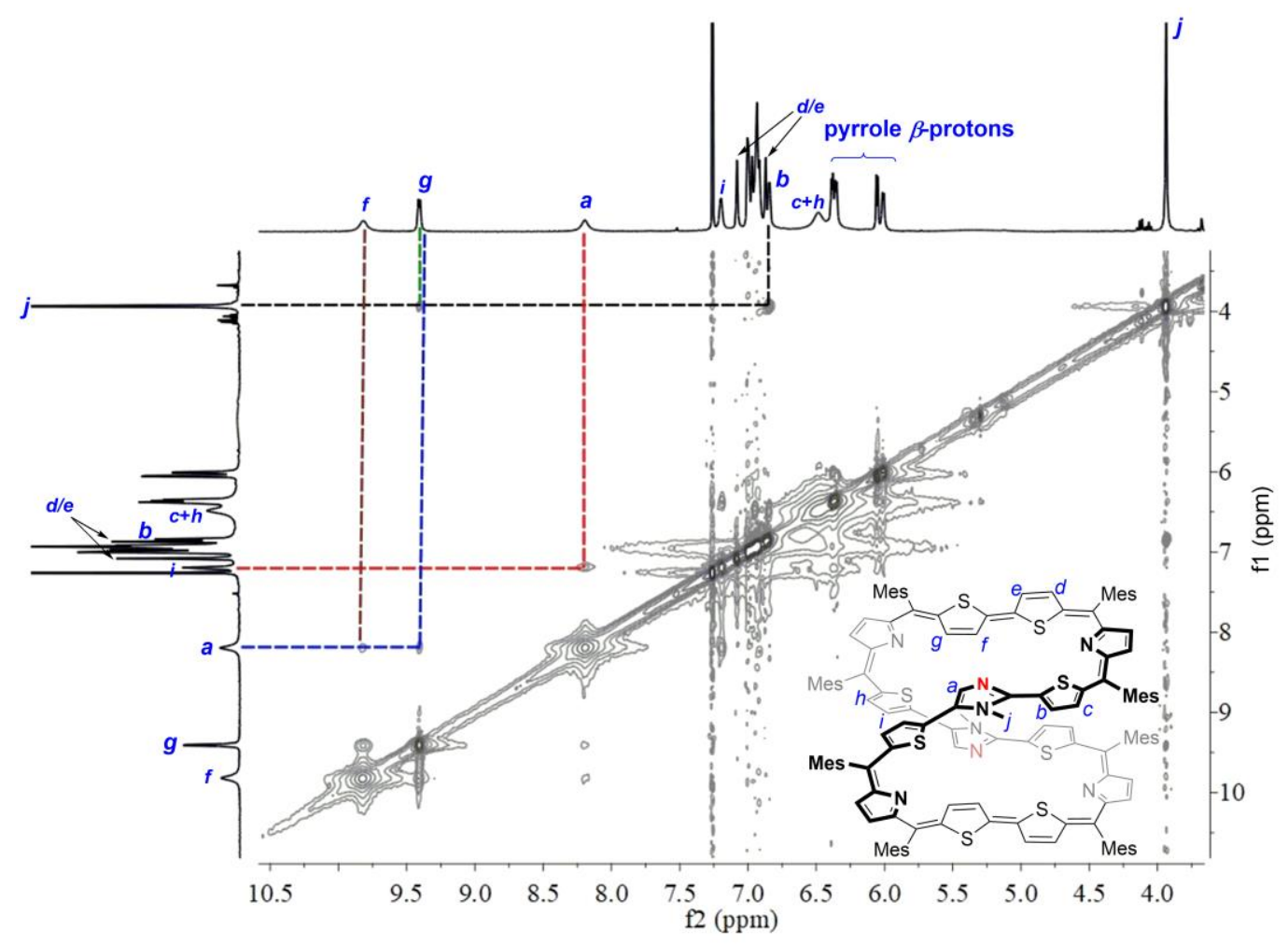

Figure S5. ${ }^{1} \mathrm{H}-{ }^{1} \mathrm{H}$ NOESY correlation spectrum of 8 at $298 \mathrm{~K}\left(400 \mathrm{MHz}, \mathrm{CDCl}_{3}\right)$.

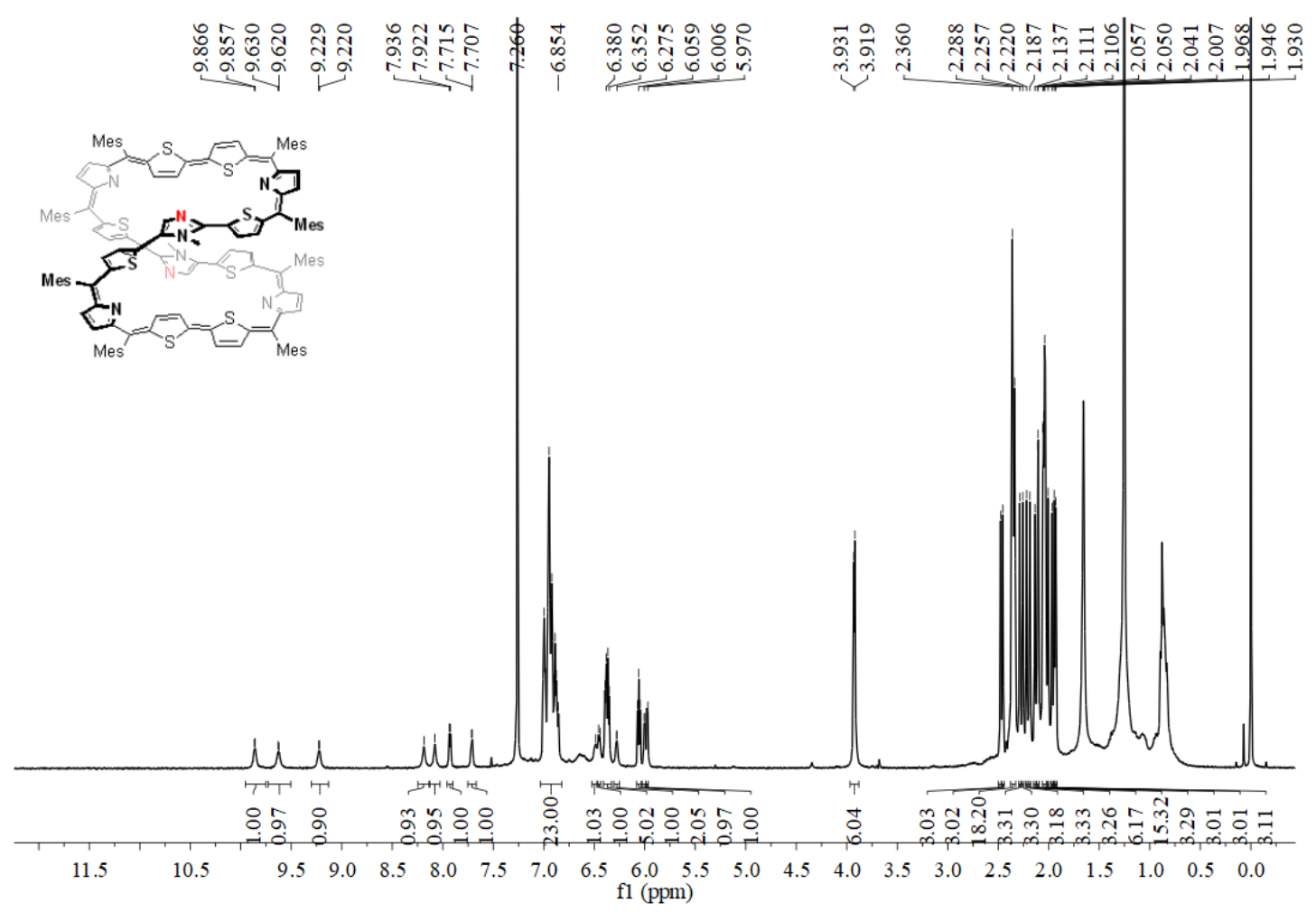

Figure S6. ${ }^{1} \mathrm{H}$ NMR spectrum of 9 at $298 \mathrm{~K}\left(400 \mathrm{MHz}, \mathrm{CDCl}_{3}\right)$. 


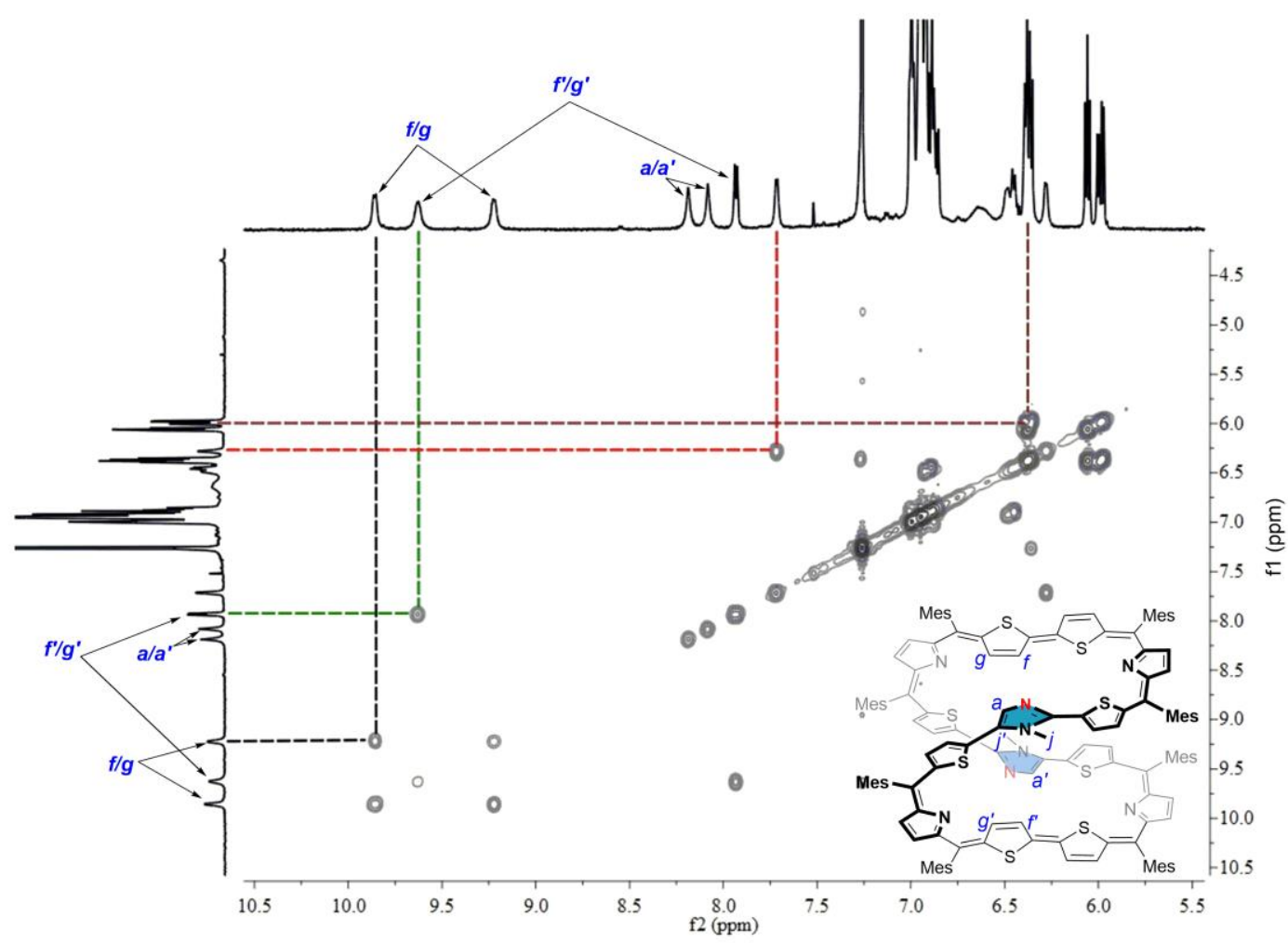

Figure S7. ${ }^{1} \mathrm{H}^{1}{ }^{1} \mathrm{H}$ COSY correlation spectrum of 9 at $298 \mathrm{~K}\left(400 \mathrm{MHz}, \mathrm{CDCl}_{3}\right)$.
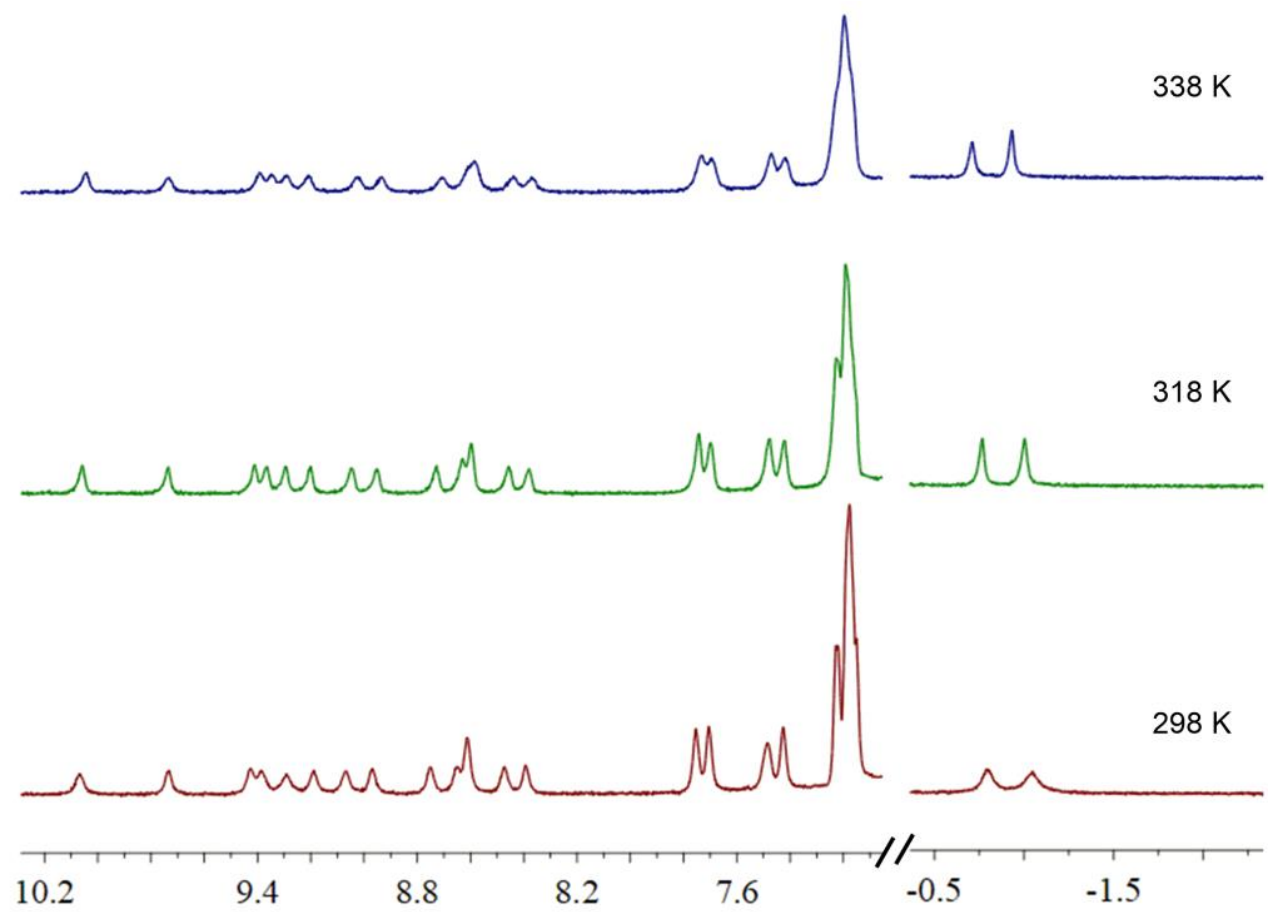

Figure S8. Variable-temperature ${ }^{1} \mathrm{H}$ NMR of 7 ( $400 \mathrm{MHz}, 1,1,2,2$-tetrachloroethane- $\left.d_{2}\right)$. 


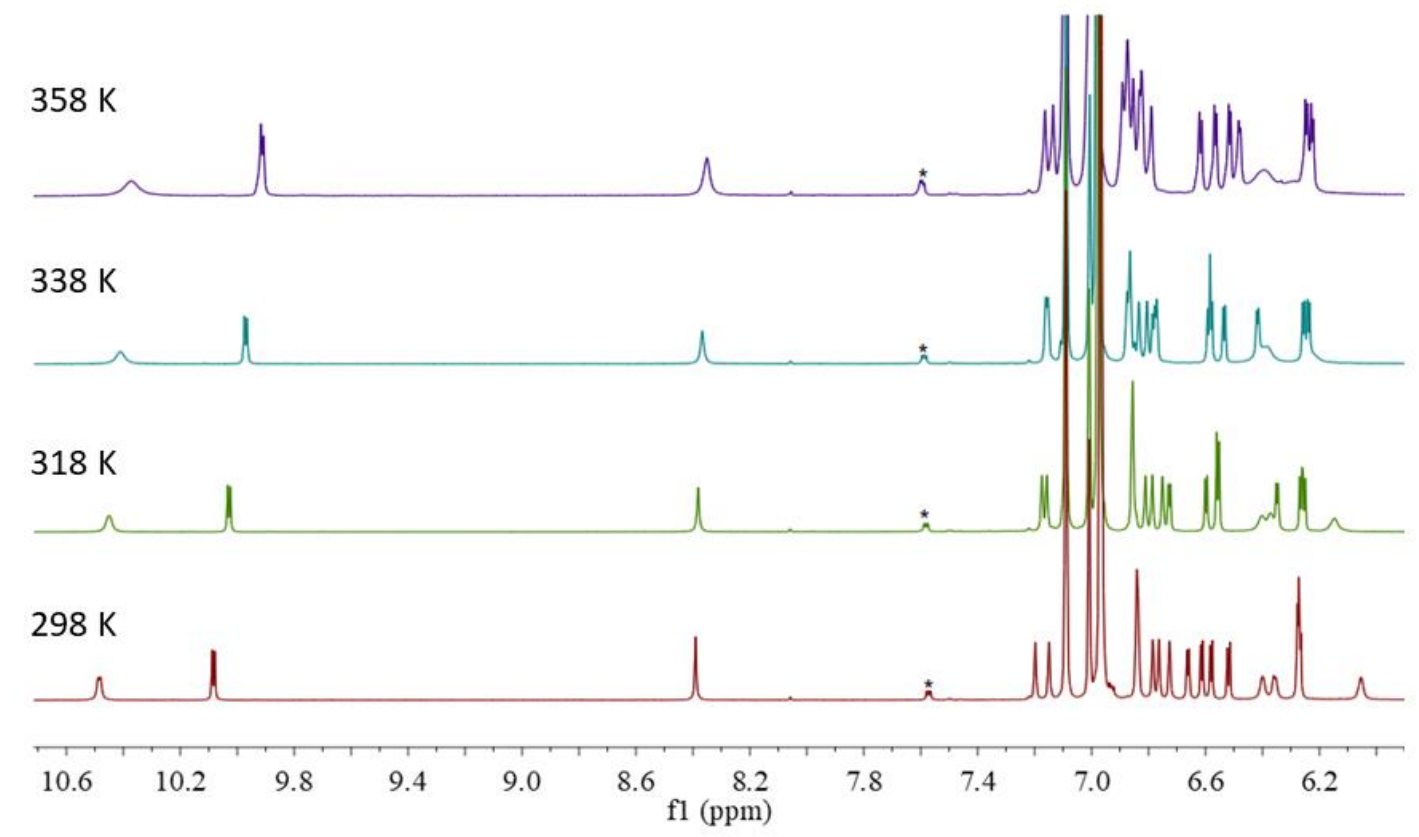

Figure S9. Variable-temperature ${ }^{1} \mathrm{H}$ NMR spectra (aromatic region) of $8\left(400 \mathrm{MHz}\right.$, toluene- $\left.d_{8}\right)$.
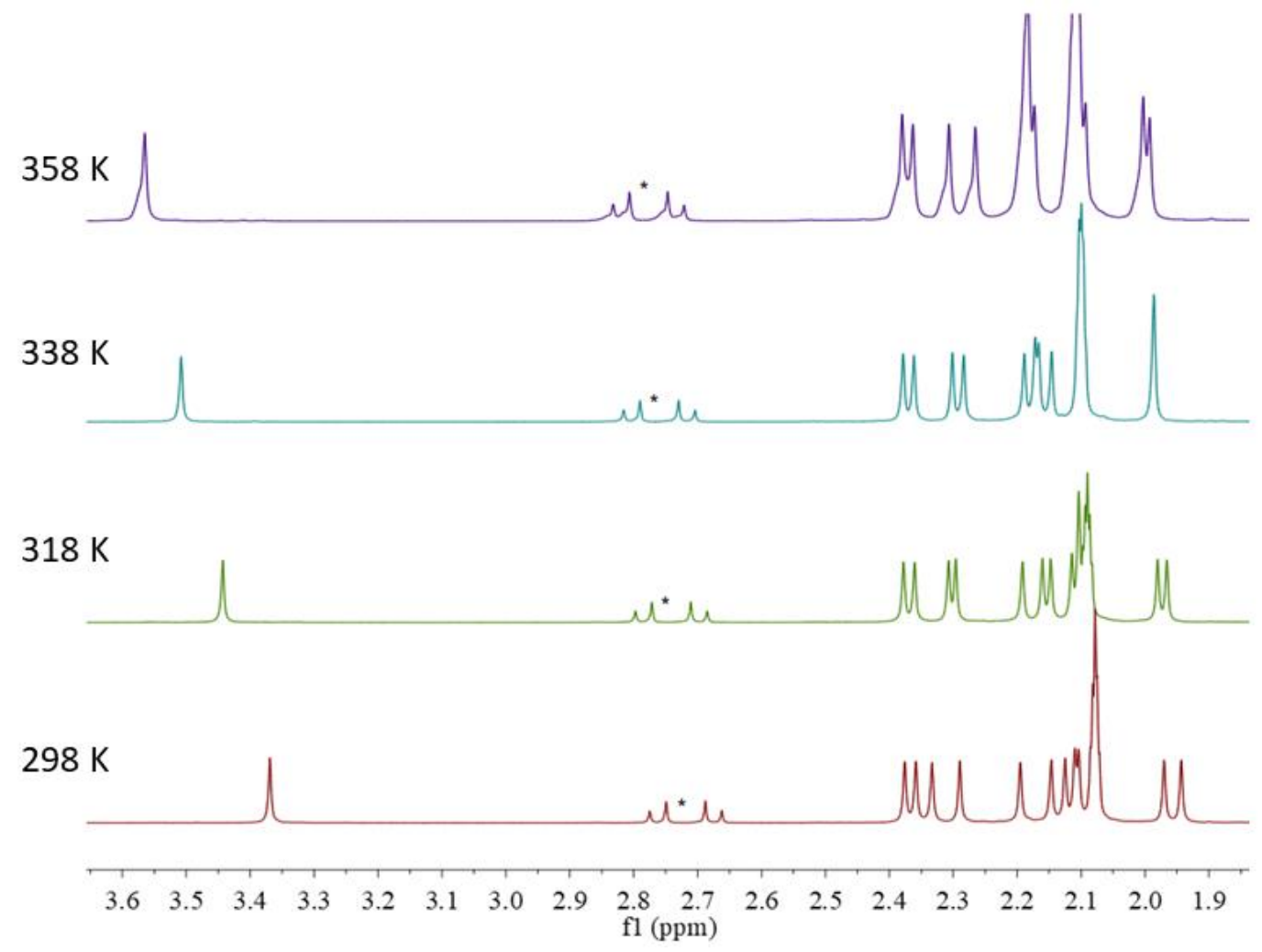

Figure S10. Variable-temperature ${ }^{1} \mathrm{H}$ NMR spectra (alkyl region) of $8\left(400 \mathrm{MHz}\right.$, toluene- $\left.d_{8}\right)$. 


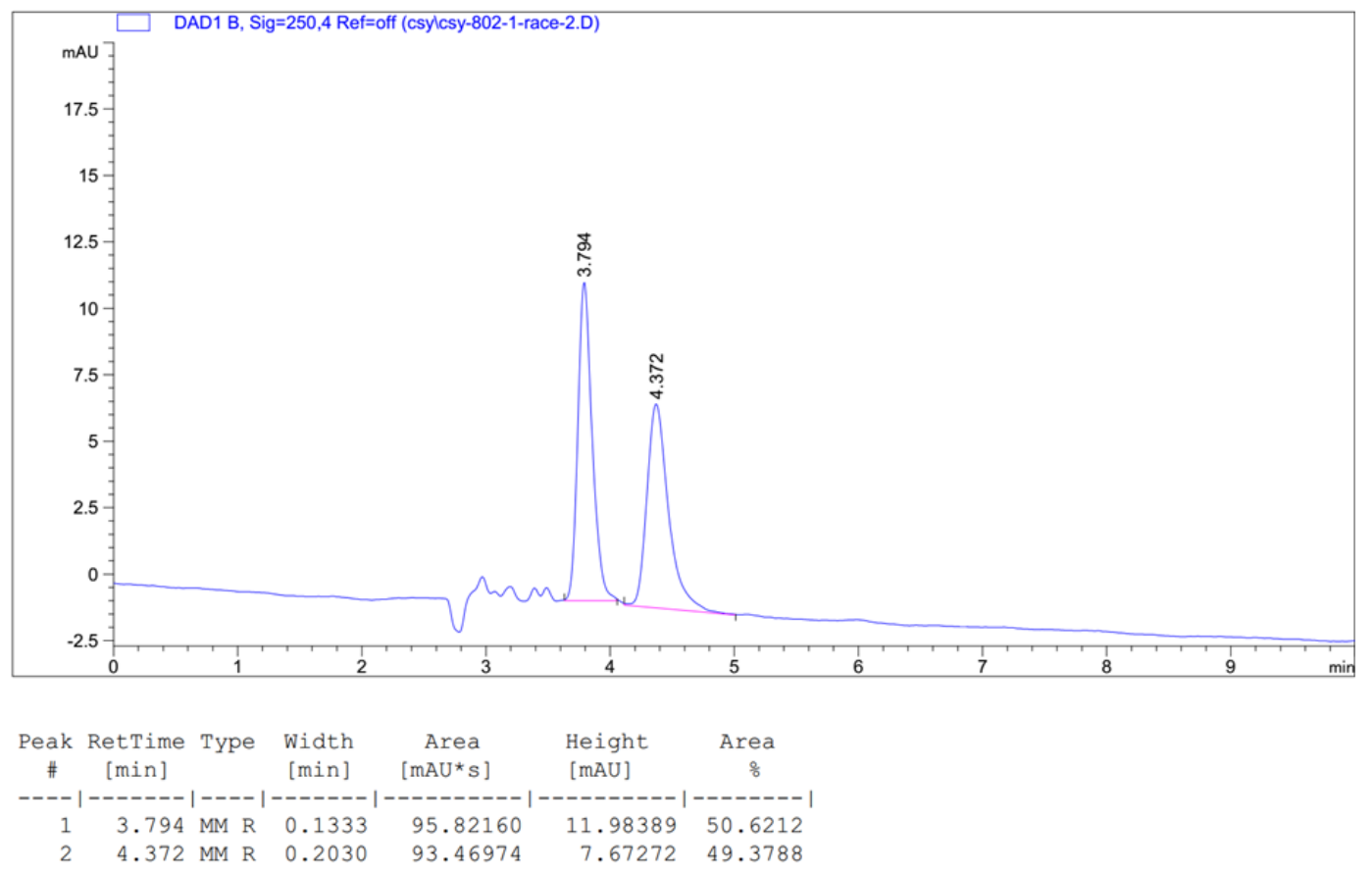

Figure S11. HPLC chart of racemic 8 (DAICEL CHIRALPACK IA, isopropanol $/ n$-hexane $=$ $20 / 80$, flow rate $=1.0 \mathrm{~mL} / \mathrm{min}, \lambda=250 \mathrm{~nm}), \mathrm{t}_{\mathrm{R}}=3.794 \mathrm{~min}$, and $4.372 \mathrm{~min}$.

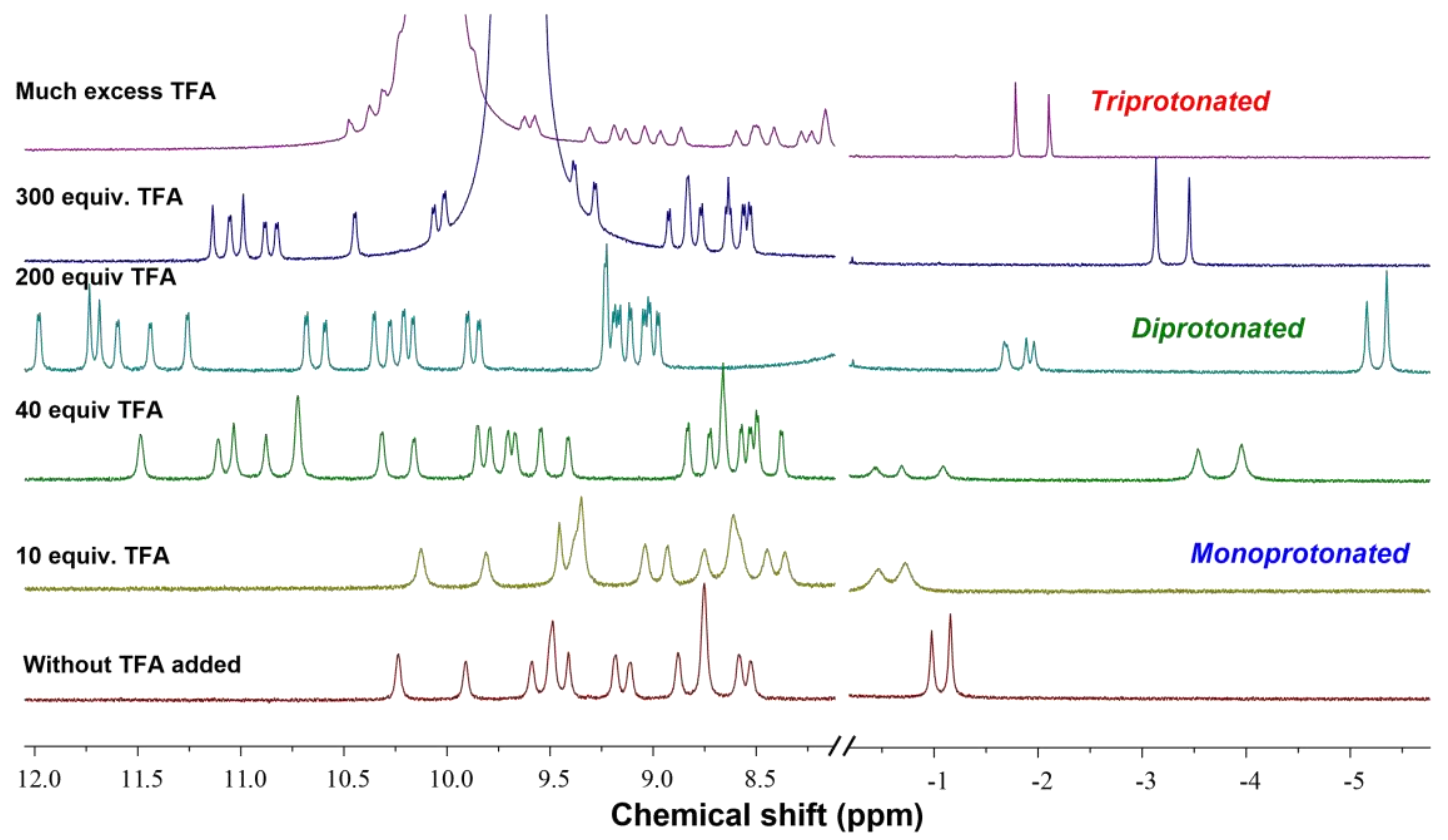

Figure S12. Change of ${ }^{1} \mathrm{H}$ NMR spectra of $7\left(400 \mathrm{MHz}, \mathrm{CDCl}_{3}\right)$ after successive addition of trifluoroacetic acid (TFA) (dissolved on a minimal amount of $\mathrm{CD}_{3} \mathrm{CN}$ ). 


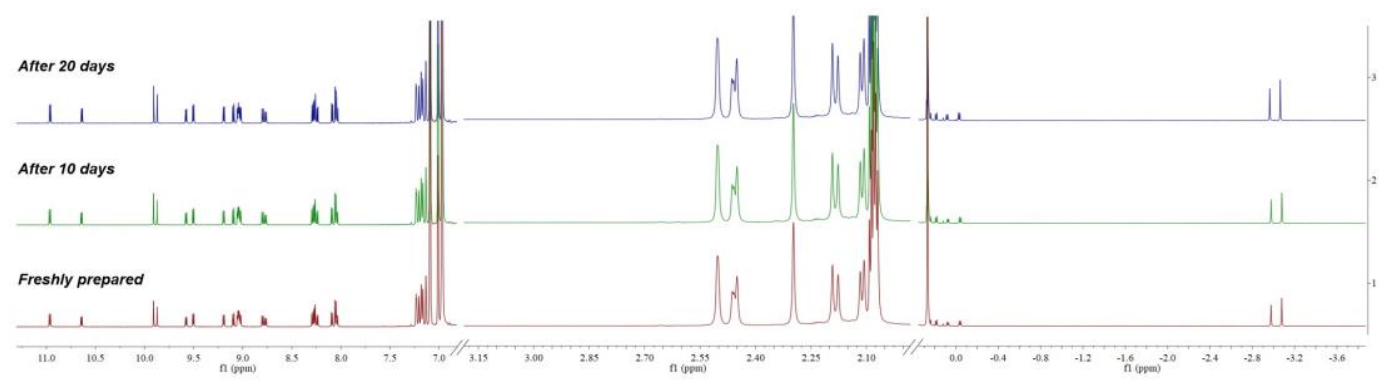

Figure S13. ${ }^{1} \mathrm{H}$ NMR spectra of 7 (400 MHz, toluene- $d_{8}$ ) of the freshly prepared solution (bottom) and the solution after stored for 20 days at $298 \mathrm{~K}$.

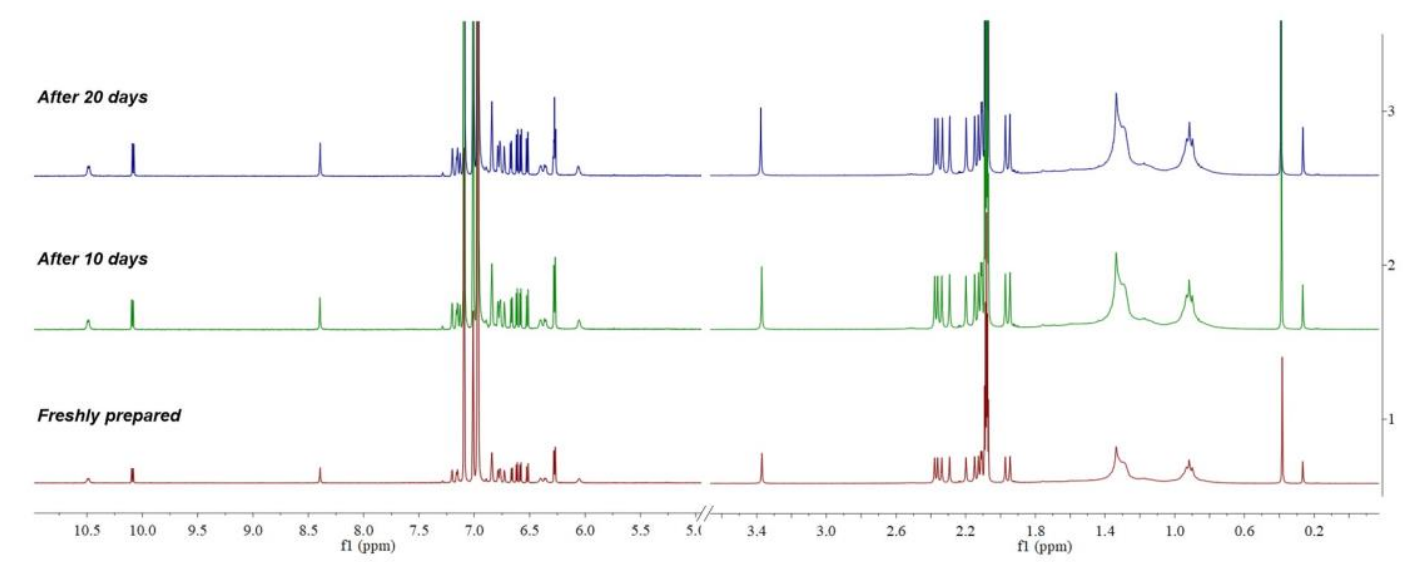

Figure S14. ${ }^{1} \mathrm{H}$ NMR spectra of $8\left(400 \mathrm{MHz}\right.$, toluene- $\left.d_{8}\right)$ of the freshly prepared solution (bottom) and the solution after stored for 20 days at $298 \mathrm{~K}$.

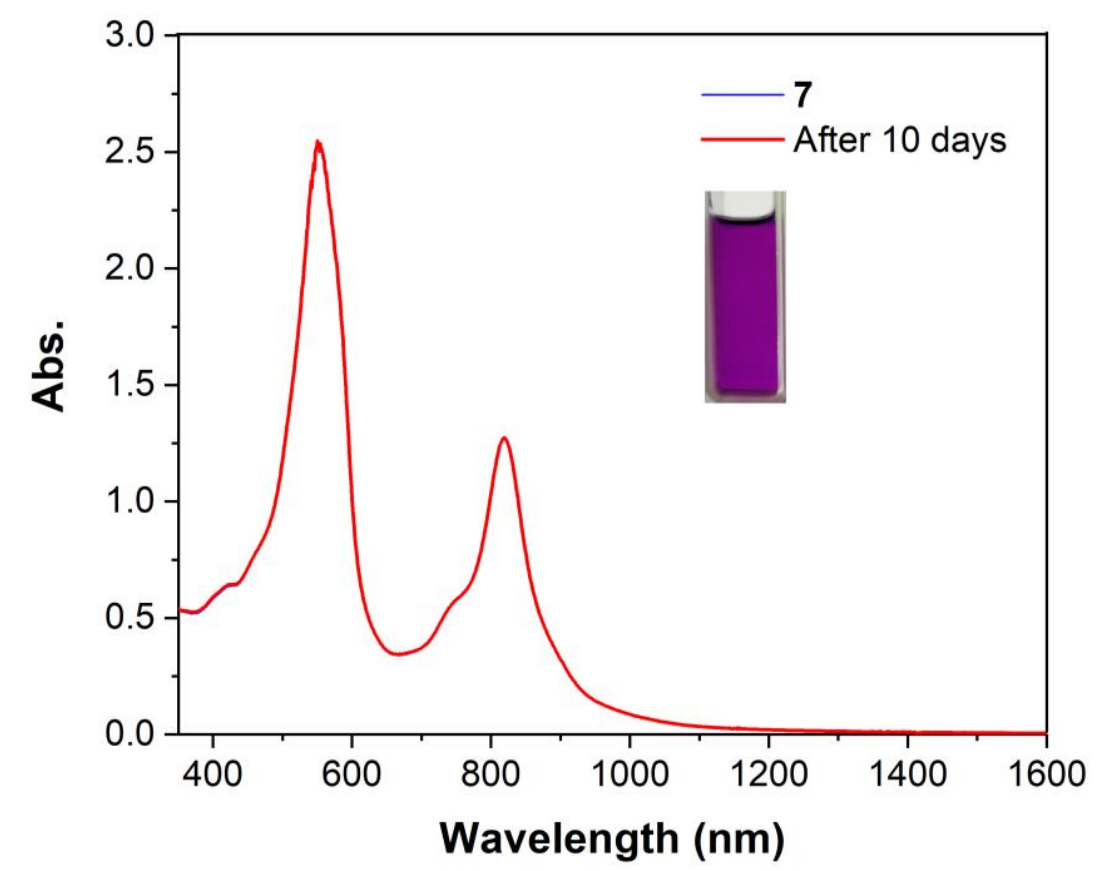

Figure S15. The photo-stability test of 7 in dry $\mathrm{CH}_{2} \mathrm{Cl}_{2}$ solution under ambient air and light conditions. The absorption spectra of the freshly prepared solution (blue line) and the solution after storing for 10 days (red line) are compared. 


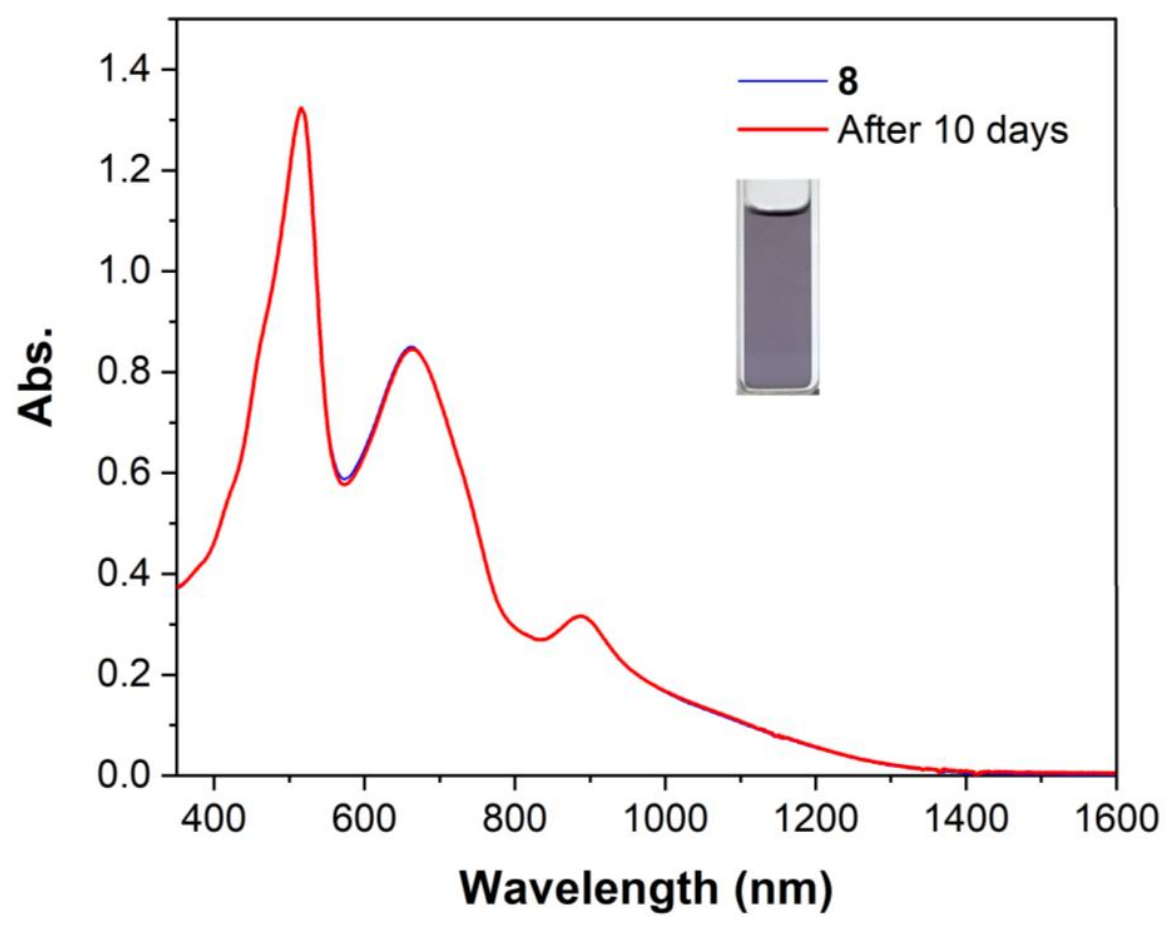

Figure S16. The photo-stability test of $\mathbf{8}$ in dry $\mathrm{CH}_{2} \mathrm{Cl}_{2}$ solution under ambient air and light conditions. The absorption spectra of the freshly prepared solution (blue line) and the solution after storing for 10 days (red line) are compared.

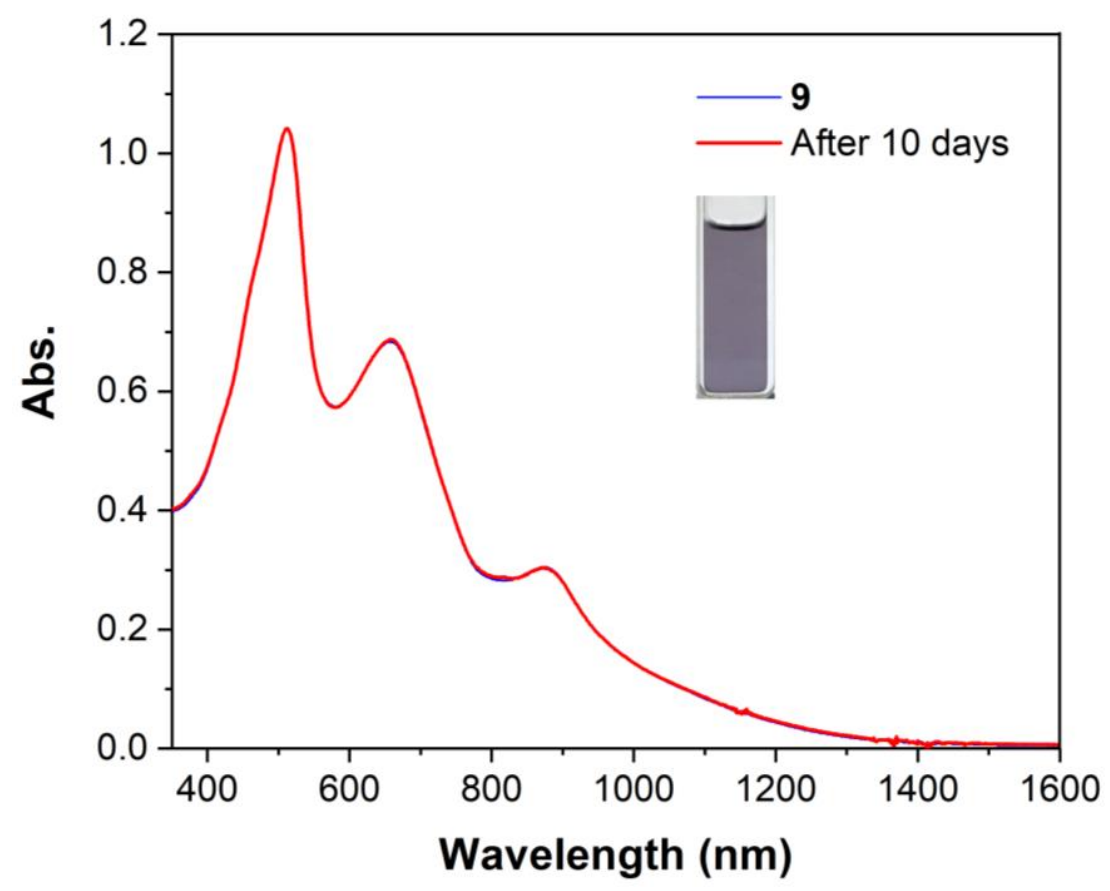

Figure S17. The photo-stability test of 9 in dry $\mathrm{CH}_{2} \mathrm{Cl}_{2}$ solution under ambient air and light conditions. The absorption spectra of the freshly prepared solution (blue line) and the solution after storing for 10 days (red line) are compared. 


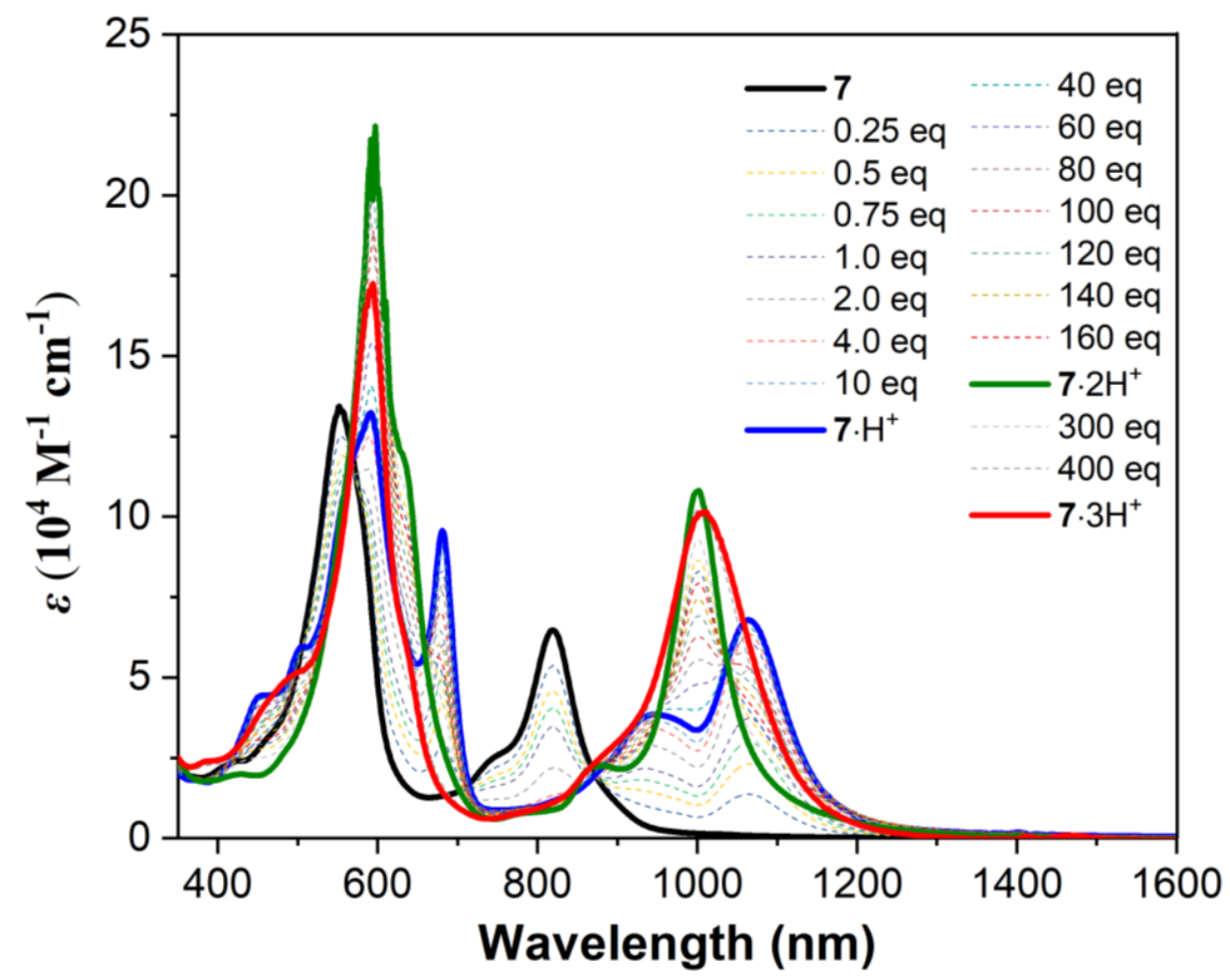

Figure S18. Variation of the UV-vis-NIR absorption spectra of $7\left(2.0 \times 10^{-5} \mathrm{M}\right)$ in $\mathrm{CH}_{2} \mathrm{Cl}_{2}$ solution during the titration with diluted TFA. $\varepsilon$ : extinction coefficient.

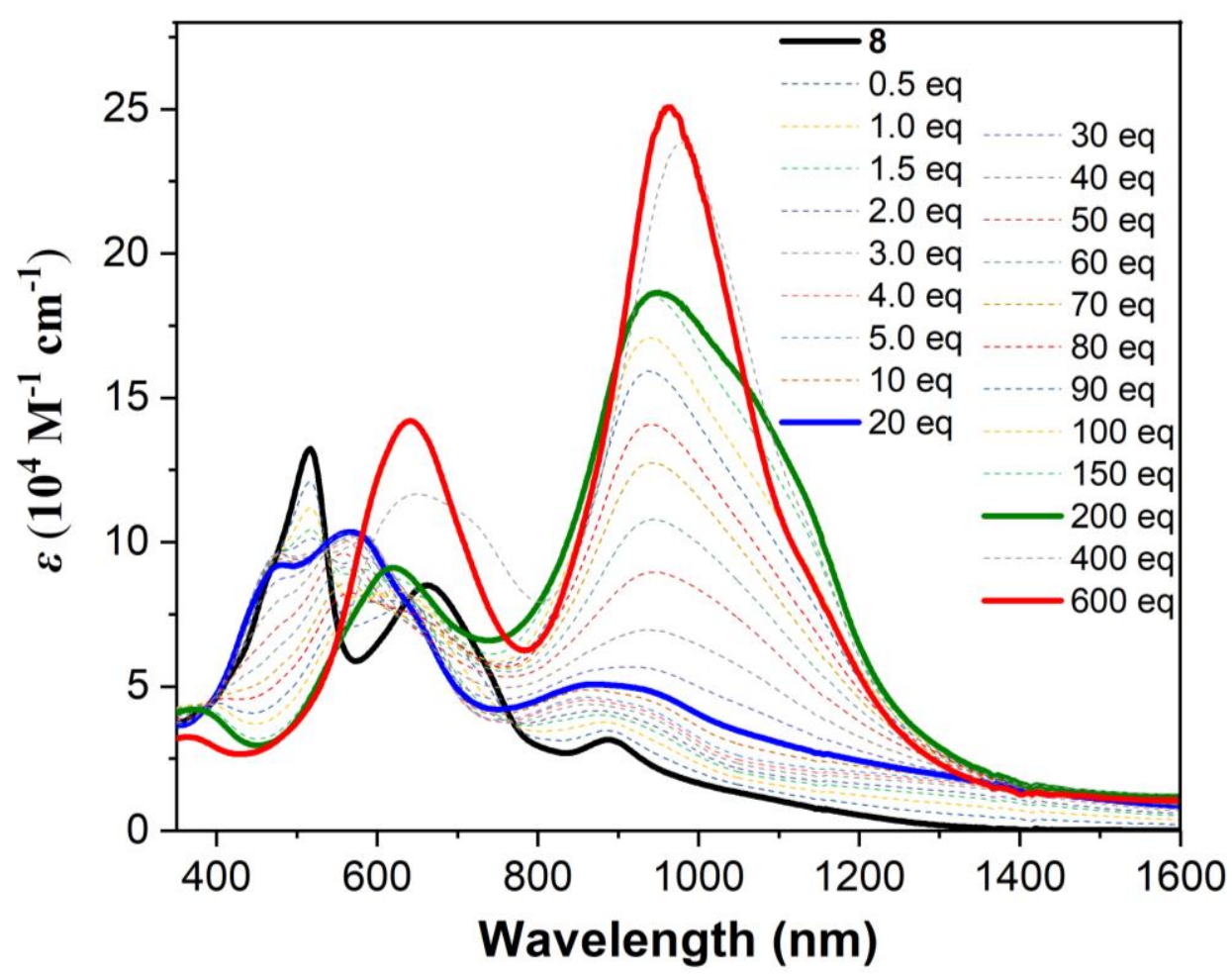

Figure S19. Variation of the UV-vis-NIR absorption spectra of $8\left(1.0 \times 10^{-5} \mathrm{M}\right)$ in $\mathrm{CH}_{2} \mathrm{Cl}_{2}$ solution during the titration with diluted TFA. $\varepsilon$ : extinction coefficient. 


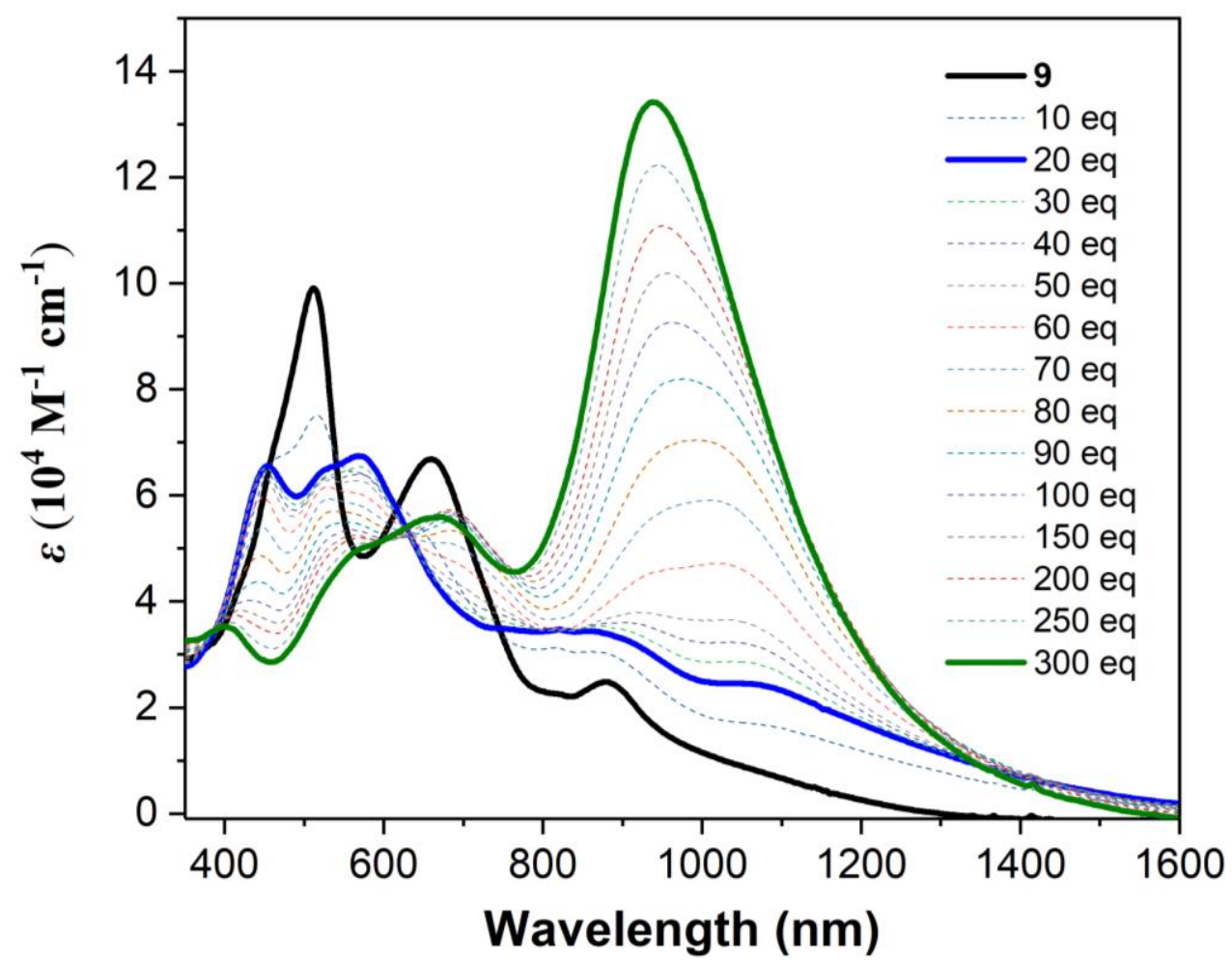

Figure S20. Variation of the UV-vis-NIR absorption spectra of $9\left(1.0 \times 10^{-5} \mathrm{M}\right)$ in $\mathrm{CH}_{2} \mathrm{Cl}_{2}$ solution during the titration with diluted TFA. $\varepsilon$ : extinction coefficient.

\section{Schematic illustration of cyclization patterns.}

Due to rotation of the $\mathrm{C}-\mathrm{C}$ single bonds, different conformers of synthons $\mathbf{4}$ and $\mathbf{5}$ are presented in the reaction system. For symmetrical $\mathbf{4}$, isomers $\mathbf{4 a}$ and $\mathbf{4 b}$ with two thiophene rings in opposite and uniform directions are representative. For unsymmetrical 5, a figure-C conformer 5a and two figure-S conformers $\mathbf{5 b} / \mathbf{c}$ are representative (Figure S21).

In the first step of acid-catalyzed cyclization, the [5+2] condensation of 5a and $\mathbf{4}$ gives macrocycle $\mathbf{4 5 a}$, while the double [5+2] condensation of $\mathbf{5 b} / \mathbf{5} \mathbf{c}$ and $\mathbf{4}$ provides higher congeners $\mathbf{4 5 b b}$ and $45 \mathrm{bc}$ with two parallel and antiparallel imidazole rings, respectively (Figure S22). The following oxidation of 45a by DDQ (Step 2) delivers [30]heptaphyrin of two conformers $\mathbf{7 a}$ and $\mathbf{7 b}$. The unfavored conformer $\mathbf{7 c}$ is not observed due to its much higher energy $(\Delta E=+20.5 \mathrm{kcal} / \mathrm{mol}$, see the Theoretical Calculations part). The oxidation of $45 \mathbf{b b}$ and $45 \mathbf{b c}$ affords racemic 
[60]tetradecaphyrins 8 and 9 in figure-eight geometry, respectively. The $N$-methyl groups should point oppositely due to the steric hindrance.

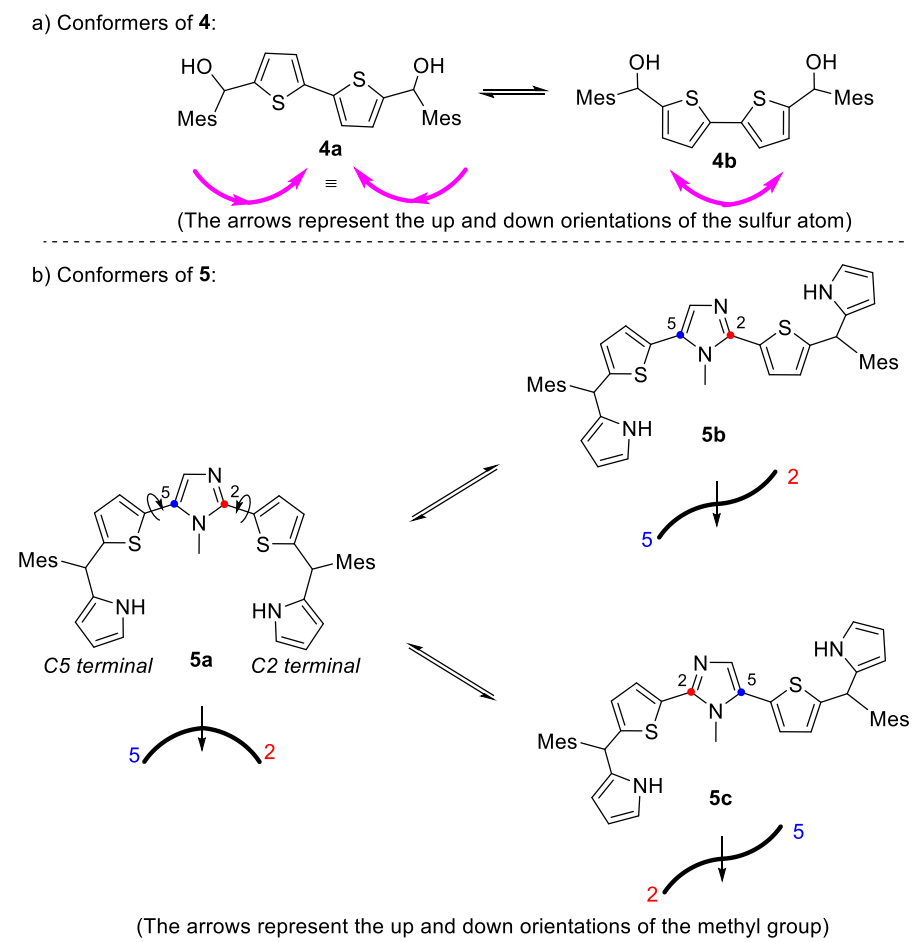

Figure S21. Schematic illustration of the conformers of $\mathbf{4}$ and 5.

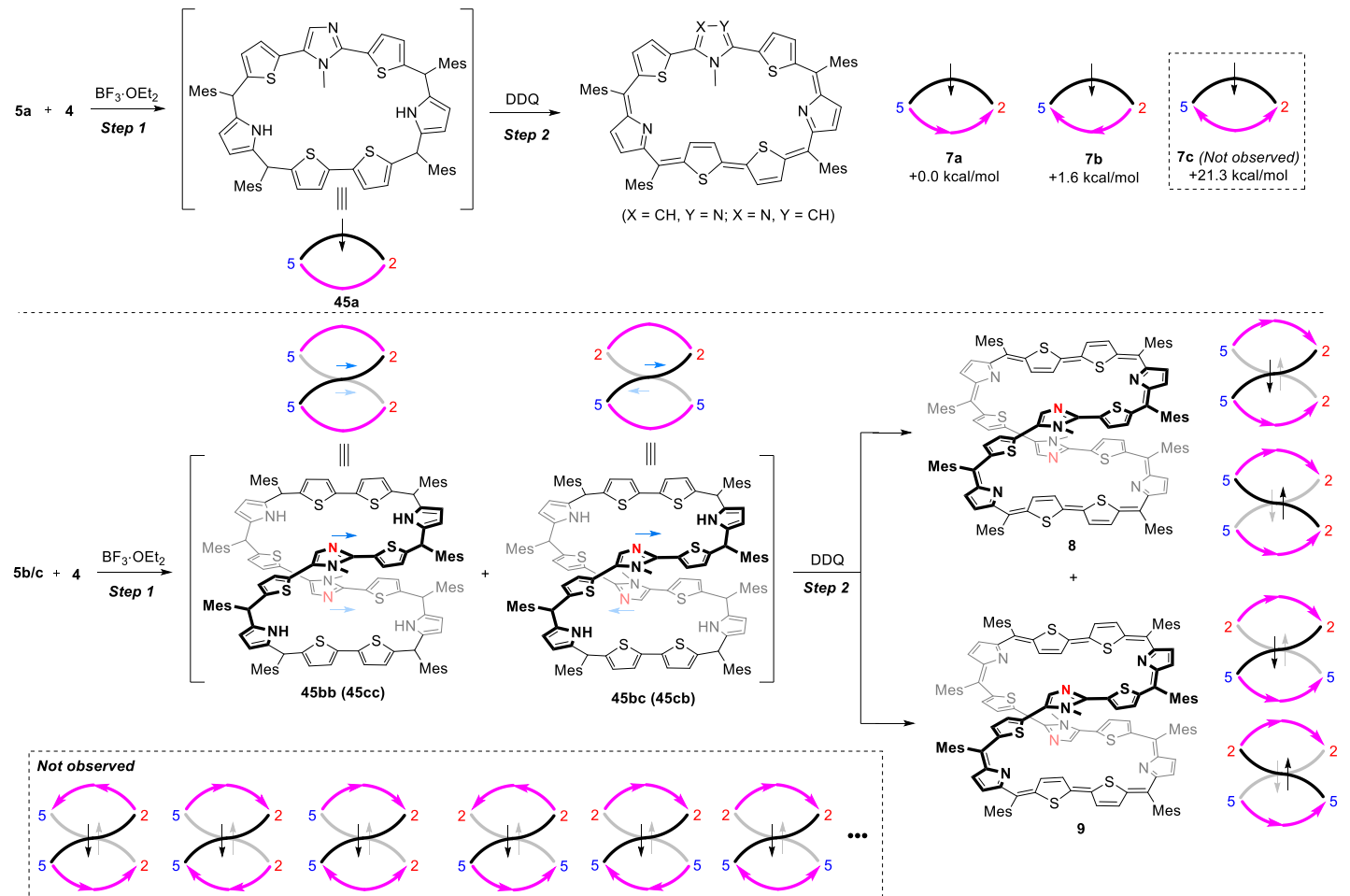

Figure S22. Cyclization patterns of $\mathbf{4}$ and $\mathbf{5}$ to form imidazole-based expanded porphyrins. 


\section{Theoretical calculations}

Theoretical calculations were carried out with the GAUSSIAN $09^{5}$ series of programs. DFT method B3-LYP ${ }^{6}$ with a standard $6-31 \mathrm{G}^{* *}$ basis set was used for all atoms. All calculations are based on optimized (B3LYP/6-31G**) geometry of 7, and the X-ray crystallographic structures of $\mathbf{8}$ and $\mathbf{9}$. Harmonic vibrational frequency calculations were performed for all of the stationary points to confirm them as a local minimum. The NICS(1) values and isosurface plots of ACID were carried out using the optimized structures. Time-dependent DFT (TD-DFT) calculations were performed at the B3LYP/6-31G** level of theory.

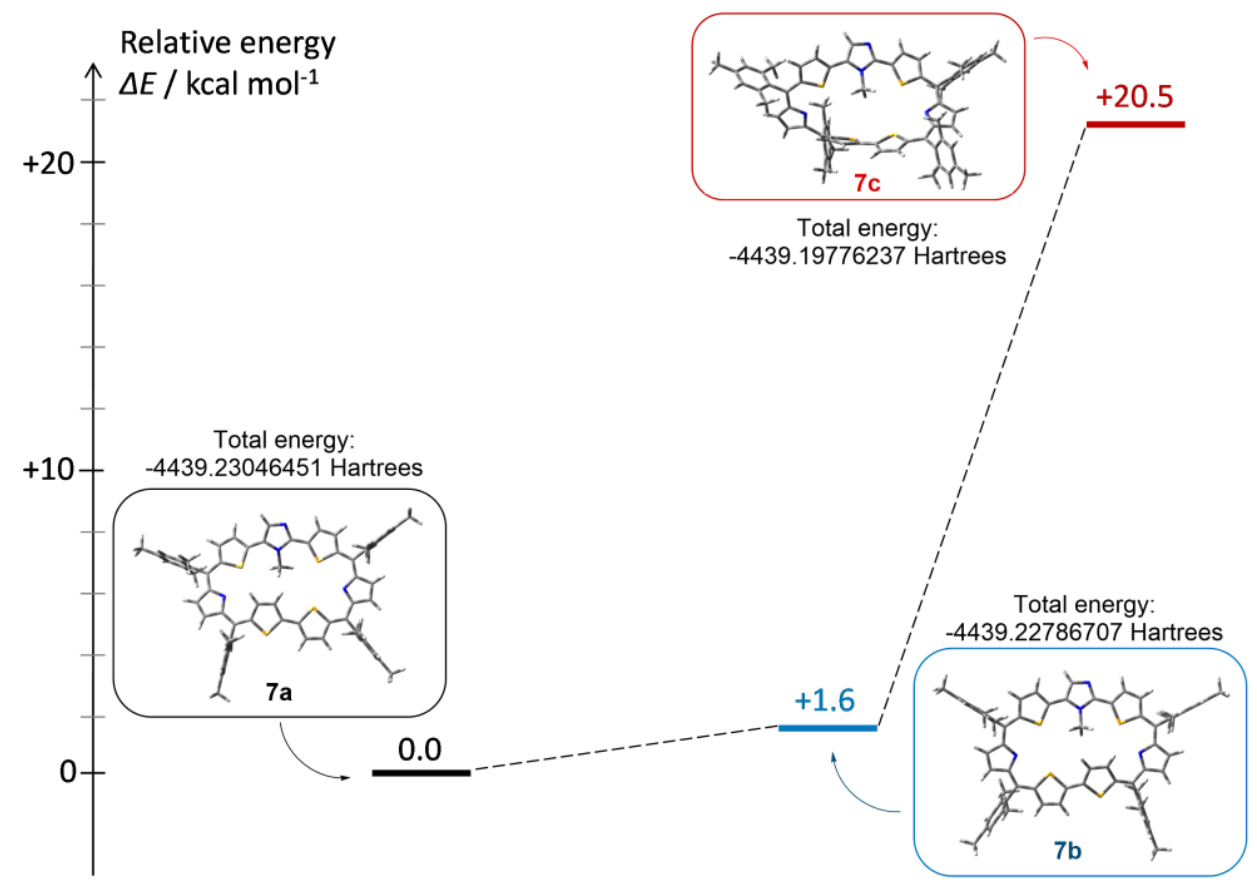

Figure S23. Optimized structures of 7 and the possible conformers (B3LYP/6-31G**). The relative energies of them $(\Delta E, \mathrm{kcal} / \mathrm{mol})$ are given based on that of $7 \mathbf{a}$. 


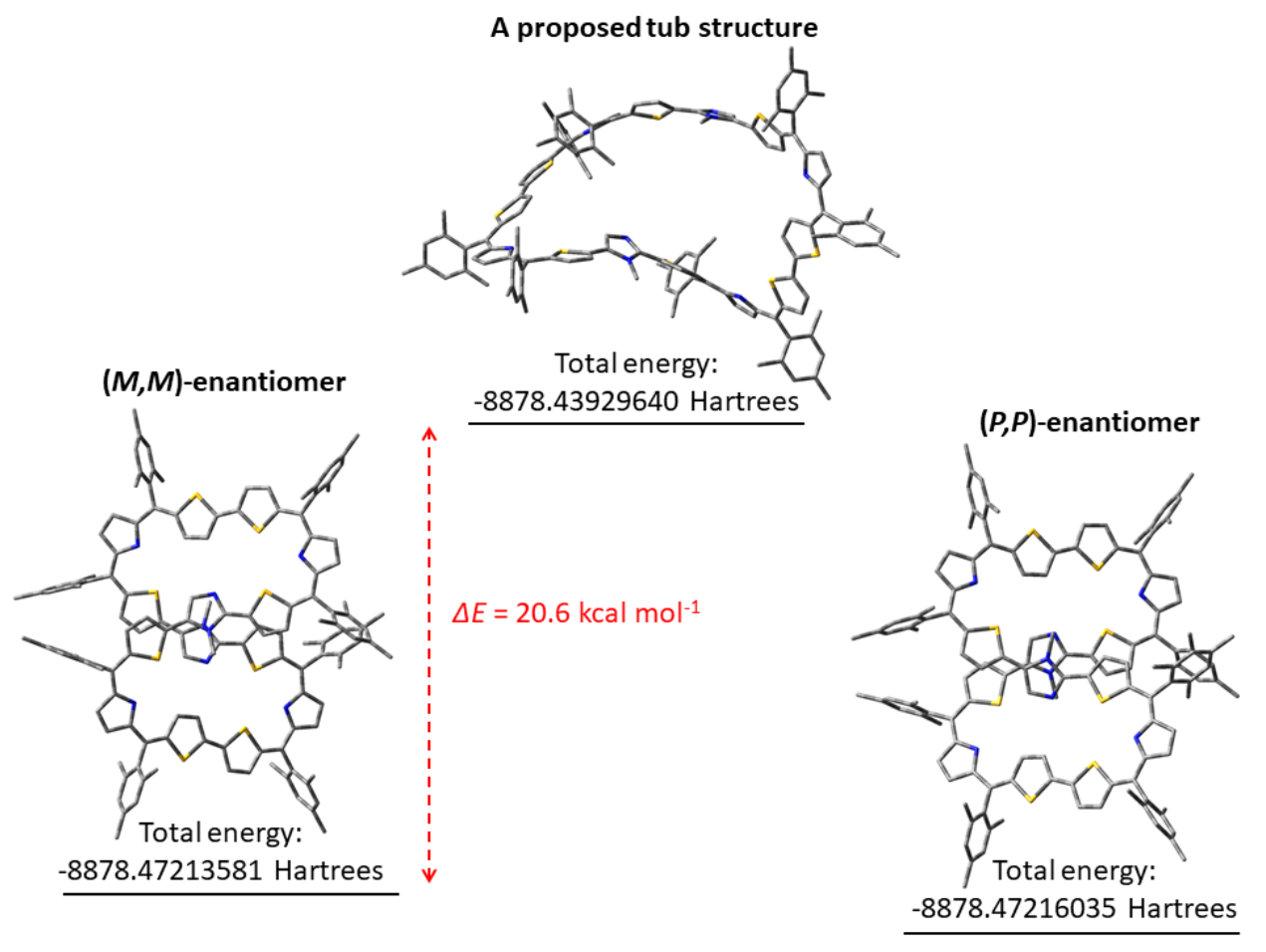

Figure S24. Optimized molecular structures of $(M, M)$ - and $(P, P)$-enantiomers of figure-eight 8 and a proposed tub conformation in interconversion. The total energies of them are calculated at the B3LYP/6-31G** level.

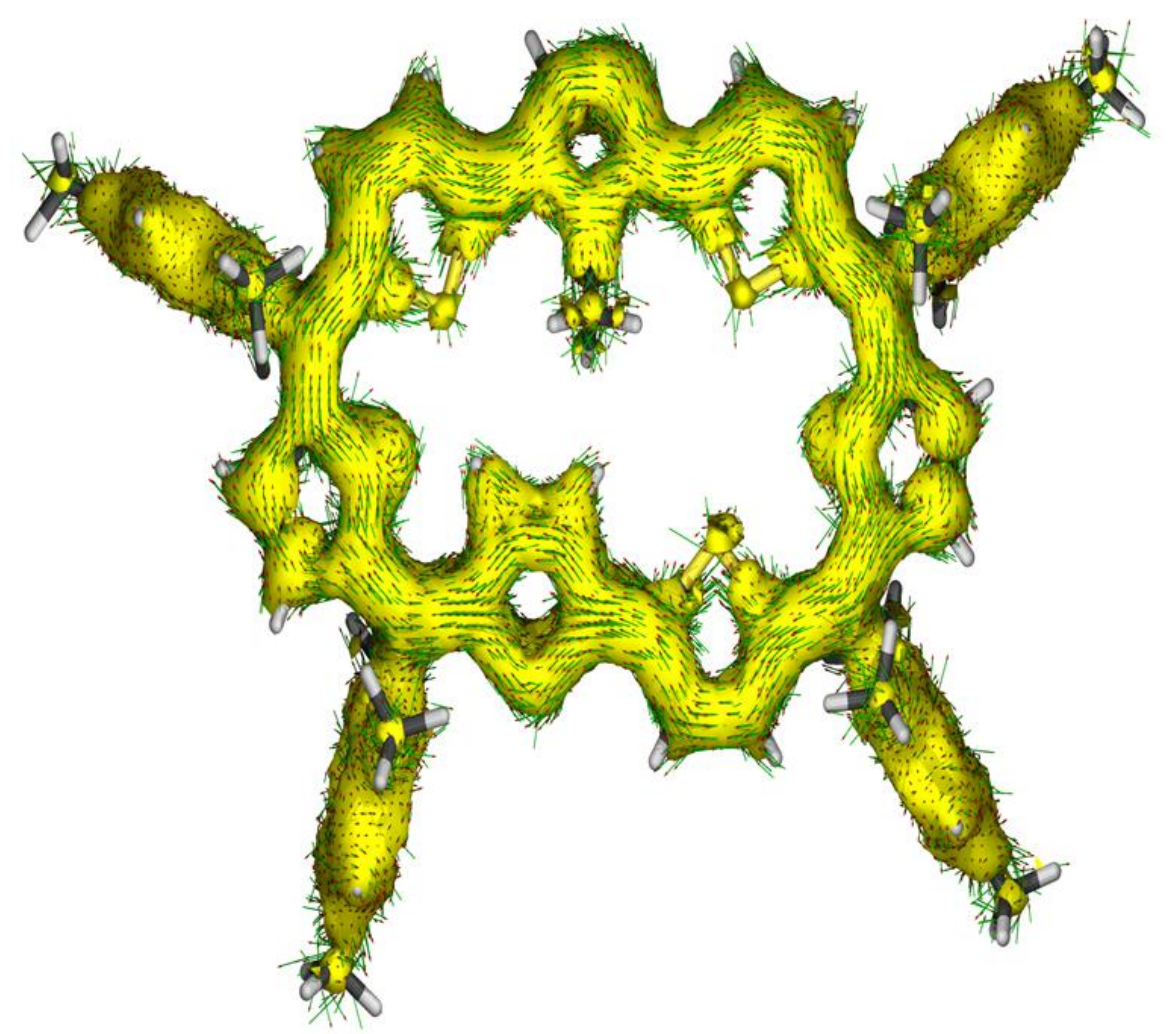

Figure S25. Calculated (B3LYP/6-31G**) ACID plots of 7a with magnetic field directed along Z axis (vertically pointing out of the paper, isovalue: 0.04 ). 


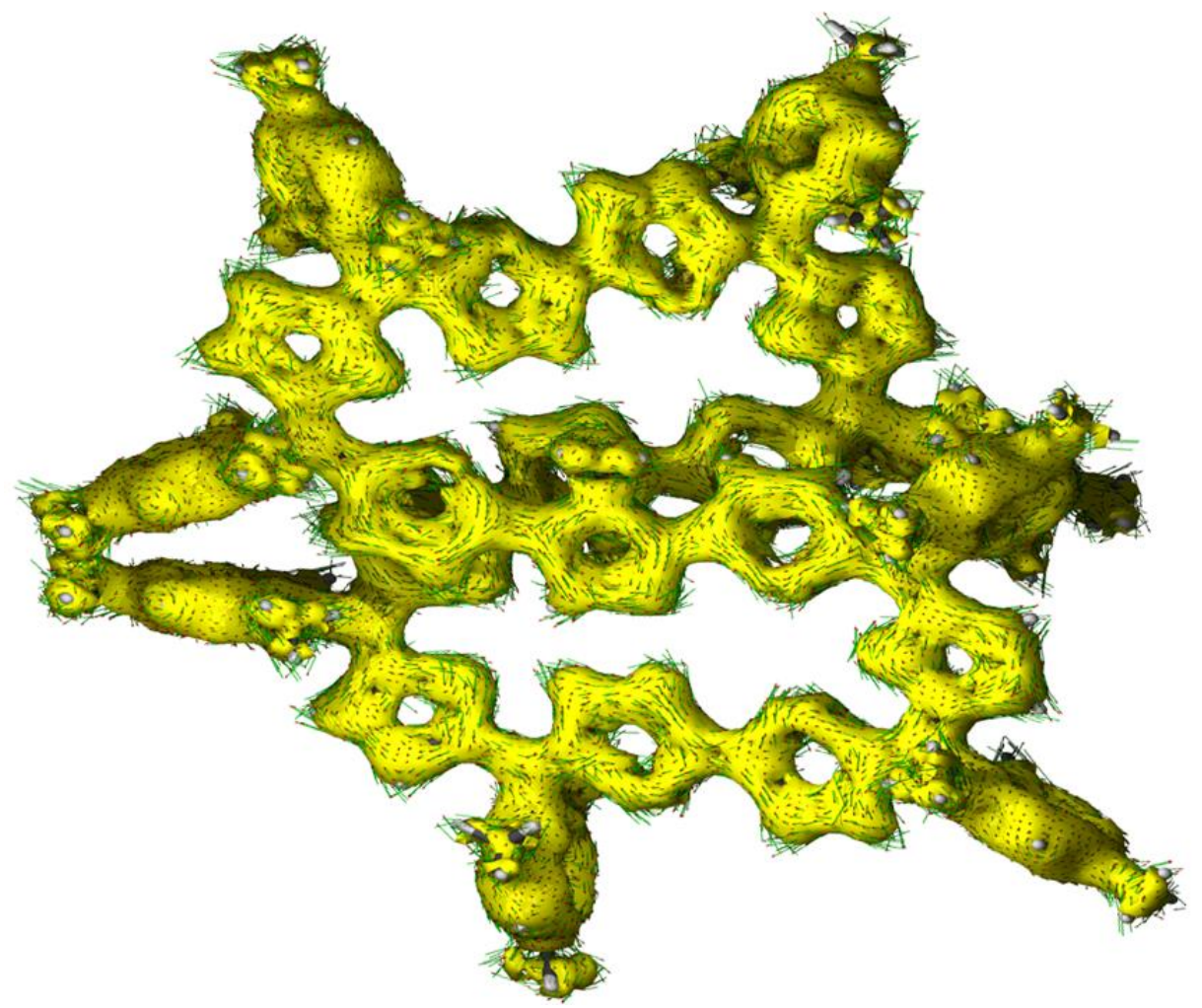

Figure S26. Calculated (B3LYP/6-31G**) ACID plots of 8 with magnetic field directed along Z axis (vertically pointing out of the paper, isovalue: 0.03 ).

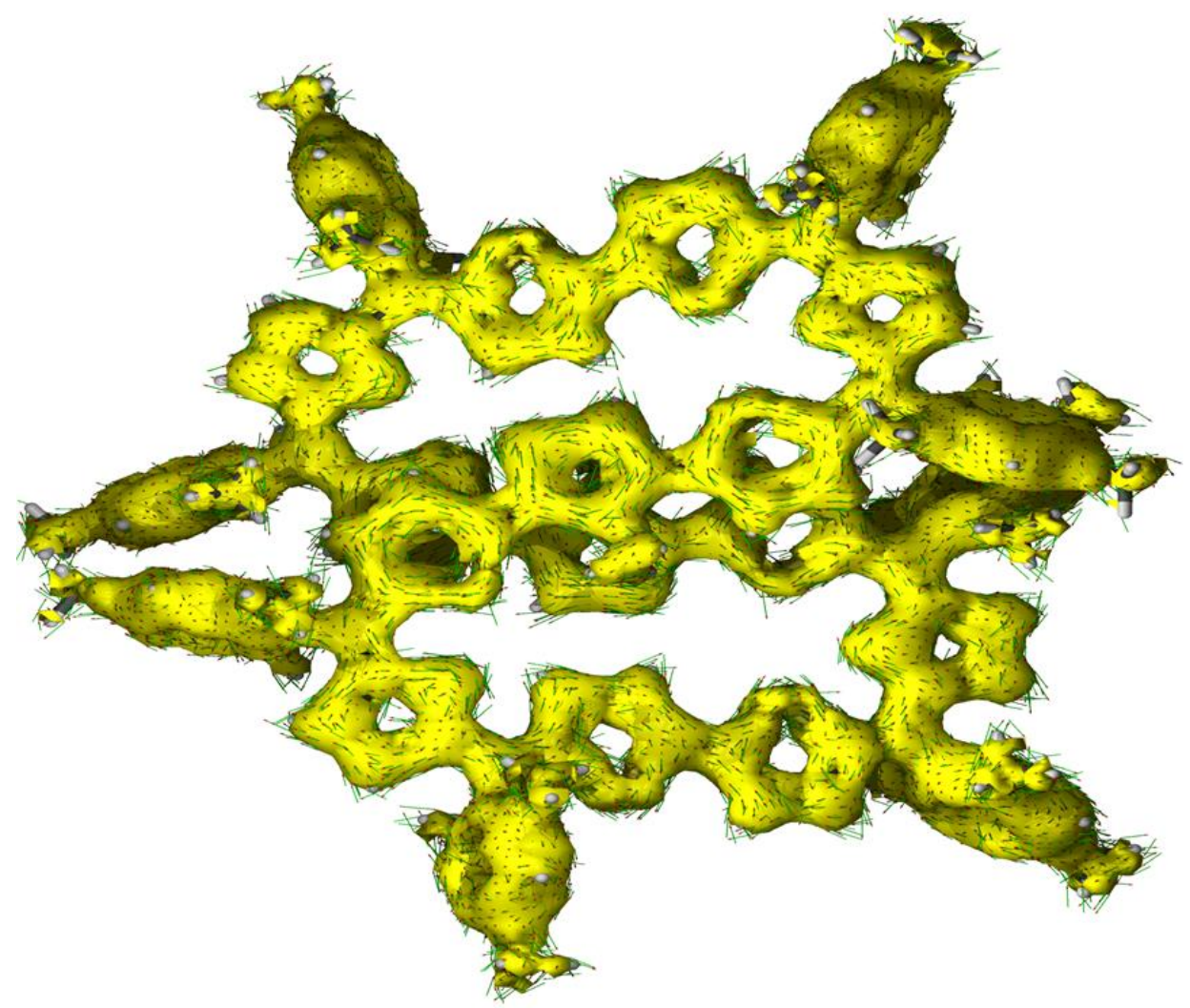

Figure S27. Calculated (B3LYP/6-31G**) ACID plots of 9 with magnetic field directed along Z axis (vertically pointing out of the paper, isovalue: 0.03 ). 


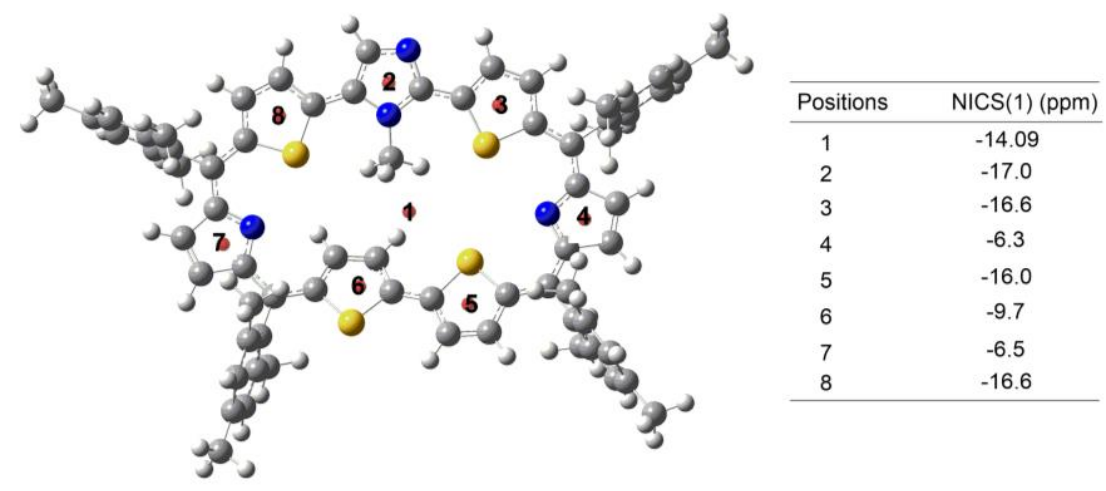

Figure S28. NICS(1) values at various positions of 7a. The GIAO method at 6-31G** level was used for calculations.

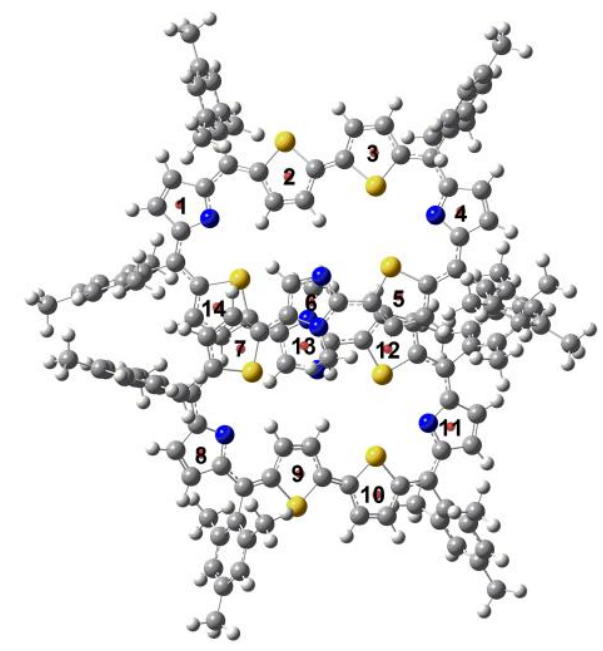

\begin{tabular}{cc}
\hline Positions & NICS(1) (ppm) \\
\hline 1 & -7.5 \\
2 & -5.8 \\
3 & -5.2 \\
4 & -6.8 \\
5 & -8.4 \\
6 & -10.1 \\
7 & -8.8 \\
8 & -6.9 \\
9 & -5.6 \\
10 & -5.4 \\
11 & -6.3 \\
12 & -8.0 \\
13 & -10.6 \\
14 & -8.0 \\
\hline
\end{tabular}

Figure S29. NICS(1) values at various positions of 8 . The GIAO method at $6-31 \mathrm{G}^{* *}$ level was used for calculations.

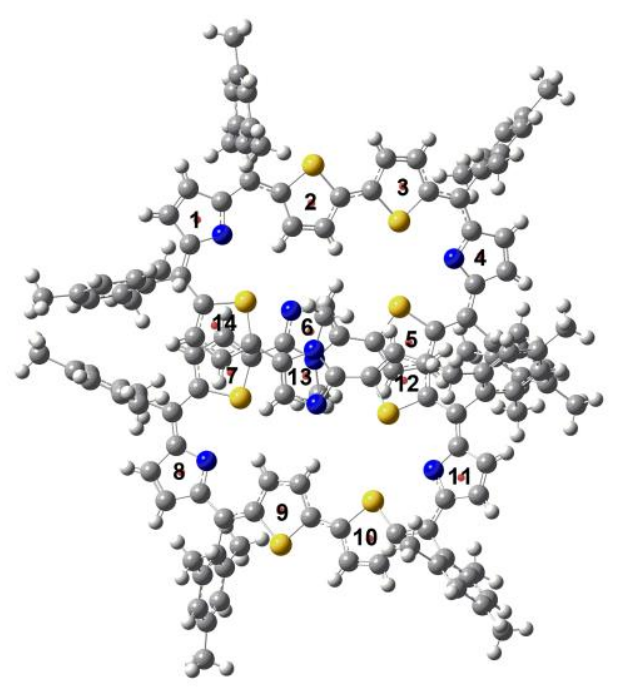

\begin{tabular}{cc}
\hline Positions & NICS(1) (ppm) \\
\hline 1 & -6.7 \\
2 & -7.0 \\
3 & -5.0 \\
4 & -6.3 \\
5 & -8.0 \\
6 & -10.4 \\
7 & -8.7 \\
8 & -6.2 \\
9 & -5.5 \\
10 & -5.2 \\
11 & -6.7 \\
12 & -8.2 \\
13 & -10.4 \\
14 & -8.6 \\
\hline
\end{tabular}

Figure S30. NICS(1) values at various positions of 9. The GIAO method at 6-31G** level was used for calculations. 


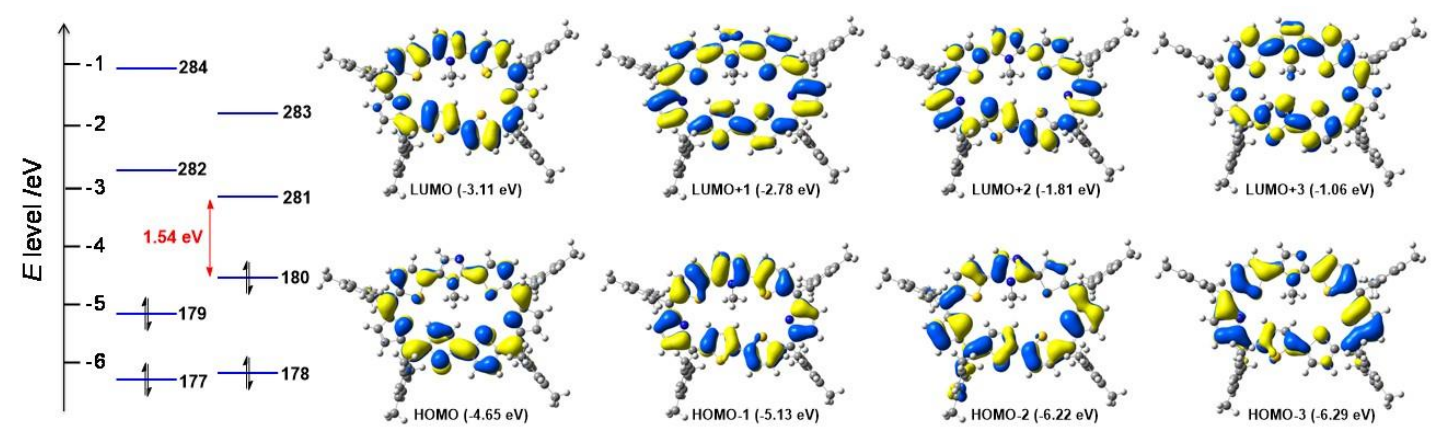

Figure S31. Frontier molecular orbital profiles and energy diagram of $7 \mathbf{a}$ obtained by B3LYP/6-31G** level calculation.

Table S3. Selected TD-DFT (B3LYP/6-31G**) calculated energies, oscillator strength and compositions of major electronic transitions of $7 \mathbf{a}$.

\begin{tabular}{|c|c|c|}
\hline Wavelength (nm) & $\begin{array}{l}\text { Oscillator strength } \\
(f)\end{array}$ & Major contributions \\
\hline 892.88 & 0.4170 & HOMO->LUMO (0.62799), HOMO-1->LUMO+1 (-0.28025) \\
\hline 881.11 & 0.0423 & HOMO->LUMO+1 (0.53561), HOMO-1->LUMO (0.42186) \\
\hline 593.02 & 1.1500 & HOMO-1->LUMO (0.56366), HOMO->LUMO+1 (-0.44587) \\
\hline 554.81 & 2.0636 & $\begin{array}{c}\text { HOMO-1->LUMO+1 (0.62075), HOMO->LUMO (0.29089), } \\
\text { HOMO->LUMO+2 (-0.19499) }\end{array}$ \\
\hline 498.64 & 0.4465 & $\begin{array}{c}\text { HOMO->LUMO+2 (0.60174), HOMO-3->LUMO (0.27057), } \\
\text { HOMO-1->LUMO+1 (0.18521) }\end{array}$ \\
\hline 455.05 & 0.0184 & HOMO-2->LUMO (0.65930), HOMO->LUMO+3 (0.14129) \\
\hline 444.96 & 0.0111 & $\begin{array}{c}\text { HOMO-4->LUMO (0.53542), HOMO-3->LUMO (0.34274), } \\
\text { HOMO-2->LUMO+1 (0.19026) }\end{array}$ \\
\hline 443.64 & 0.0290 & $\begin{array}{l}\text { HOMO-4->LUMO (0.40203), HOMO-3->LUMO (-0.39099), } \\
\text { HOMO-2->LUMO+1 (-0.32795), HOMO->LUMO+2 (0.19792) }\end{array}$ \\
\hline 440.05 & 0.0057 & HOMO-5->LUMO (0.67480), HOMO-5->LUMO+1 (0.11772) \\
\hline 438.07 & 0.0005 & HOMO-6->LUMO (0.66061), HOMO-5->LUMO (-0.17549) \\
\hline 434.40 & 0.0005 & HOMO-7->LUMO (0.67029), HOMO-7->LUMO+1 (0.13227) \\
\hline 426.51 & 0.0146 & HOMO-1->LUMO+2 (0.61815), HOMO->LUMO+3 (0.31333) \\
\hline 422.29 & 0.0015 & HOMO-8->LUMO (0.69718) \\
\hline 421.50 & 0.0007 & HOMO-9->LUMO (0.69934) \\
\hline 420.50 & 0.0007 & HOMO-10->LUMO (0.67666), HOMO-10->LUMO+1 (-0.17102) \\
\hline 418.35 & 0.0001 & HOMO-11->LUMO (0.68097), HOMO-11->LUMO+1 (0.17666) \\
\hline 412.90 & 0.0384 & HOMO-12->LUMO (0.65866), HOMO-2->LUMO+1 (0.18591) \\
\hline 406.15 & 0.0464 & $\begin{array}{c}\text { HOMO-3->LUMO+1 (0.47793), HOMO-13->LUMO (-0.39088), } \\
\text { HOMO-12->LUMO+1 (-0.17024) }\end{array}$ \\
\hline 405.03 & 0.0947 & $\begin{array}{c}\text { HOMO-2->LUMO+1 (0.47166), HOMO-3->LUMO (-0.28826), } \\
\text { HOMO-13->LUMO+1 (-0.20945) }\end{array}$ \\
\hline 394.53 & 0.0007 & HOMO-4->LUMO+1 (0.65741), HOMO-3->LUMO+1 (0.16548) \\
\hline
\end{tabular}



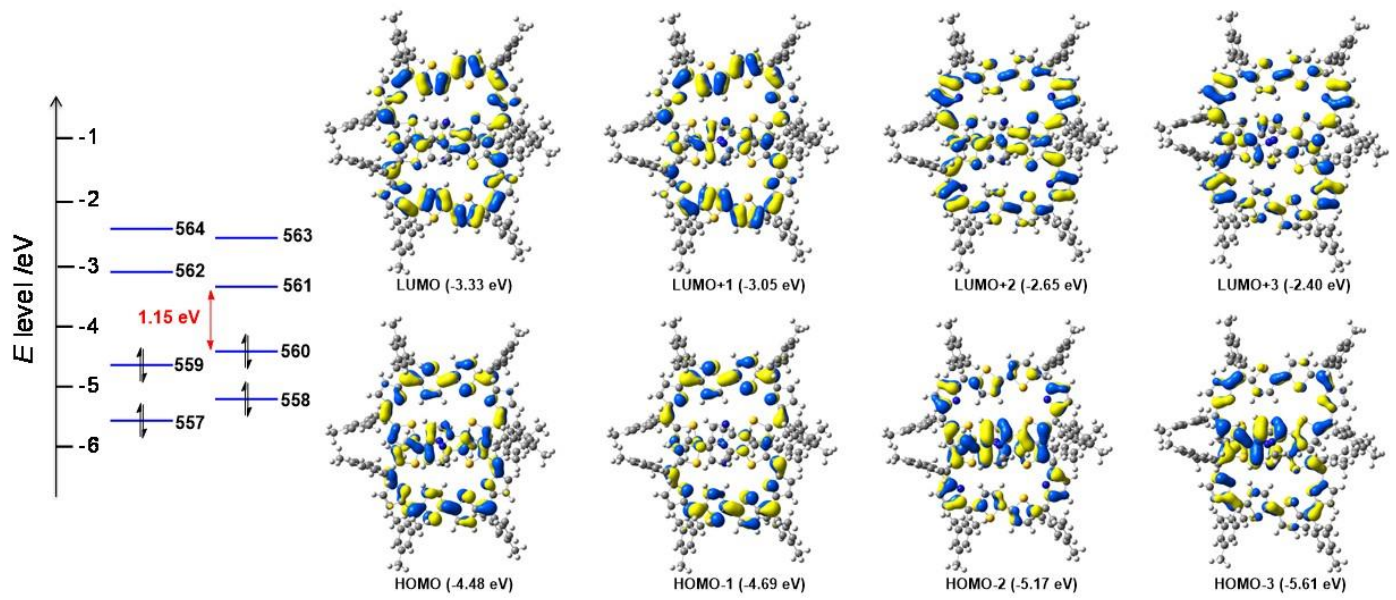

Figure S32. Frontier molecular orbital profiles and energy diagram of $\mathbf{8}$ obtained by B3LYP/6-31G** level calculation.

Table S4. Selected TD-DFT (B3LYP/6-31G**) calculated energies, oscillator strength and compositions of major electronic transitions of $\mathbf{8}$.

\begin{tabular}{|c|c|c|}
\hline $\begin{array}{l}\text { Wavelength } \\
(\mathrm{nm})\end{array}$ & Oscillator strength (f) & Major contributions \\
\hline 1548.67 & 0.0803 & HOMO->LUMO (0.70284) \\
\hline 1134.06 & 0.0112 & HOMO-1->LUMO (0.58812), HOMO->LUMO+1 (-0.39056) \\
\hline 1000.50 & 0.2926 & HOMO->LUMO+1 (0.59208), HOMO-1->LUMO (0.39629) \\
\hline 870.68 & 0.3861 & $\begin{array}{c}\text { HOMO-1->LUMO+1 (0.68693), HOMO-2->LUMO+2 } \\
(0.11691), \text { HOMO-3->LUMO (0.10947) }\end{array}$ \\
\hline 857.70 & 0.0034 & HOMO-2->LUMO (0.52560), HOMO->LUMO+2 (-0.46490) \\
\hline 758.71 & 1.4379 & HOMO->LUMO+2 (0.52747), HOMO-2->LUMO (0.46481) \\
\hline 748.82 & 0.0001 & $\begin{array}{c}\text { HOMO-2->LUMO+1 (0.46967), HOMO-1->LUMO+2 } \\
(-0.43982), \text { HOMO->LUMO+3 (-0.26309) }\end{array}$ \\
\hline 703.47 & 0.0002 & $\begin{array}{c}\text { HOMO->LUMO+3 }(0.53532), \text { HOMO-1->LUMO+2 (-0.44282), } \\
\text { HOMO-2->LUMO+1 (-0.12050) }\end{array}$ \\
\hline 642.96 & 0.1053 & $\begin{array}{c}\text { HOMO-3->LUMO (0.41921), HOMO-2->LUMO+1 (0.35841), } \\
\text { HOMO-4->LUMO (0.26609), HOMO->LUMO+3 (0.25151), } \\
\text { HOMO-1->LUMO+2 }(0.17251)\end{array}$ \\
\hline 631.83 & 0.1260 & $\begin{array}{c}\text { HOMO-3->LUMO (0.48726), HOMO-2->LUMO+1 (-0.29445), } \\
\text { HOMO-4->LUMO (-0.27742) }\end{array}$ \\
\hline 621.35 & 0.2673 & HOMO-1->LUMO+3 (0.67246), HOMO-4->LUMO+1 (0.16857) \\
\hline 579.27 & 0.0086 & HOMO-2->LUMO+2 (0.51860), HOMO->LUMO+4 (-0.46392) \\
\hline 563.88 & 0.0196 & $\begin{array}{c}\text { HOMO-3->LUMO+1 (0.62503), HOMO->LUMO+5 (0.22038), } \\
\text { HOMO-1->LUMO+4 (-0.20352) }\end{array}$ \\
\hline 547.86 & 0.0058 & $\begin{array}{l}\text { HOMO-4->LUMO (0.56947), HOMO-1->LUMO+2 (-0.24592), } \\
\text { HOMO->LUMO+3 (-0.22760), HOMO-2->LUMO+1 (-0.22068) }\end{array}$ \\
\hline 530.56 & 0.0170 & $\begin{array}{c}\text { HOMO-2->LUMO+3 (0.45746), HOMO-1->LUMO+4 } \\
(-0.34919), \text { HOMO->LUMO+5 (0.27339), HOMO-3->LUMO+1 } \\
(-0.19789)\end{array}$ \\
\hline 516.39 & 0.5152 & $\begin{array}{c}\text { HOMO-4->LUMO+1 (0.62447), HOMO-3->LUMO+2 } \\
(-0.24189), \text { HOMO-1->LUMO+3 }(-0.15550)\end{array}$ \\
\hline 514.26 & 2.8796 & $\begin{array}{c}\text { HOMO->LUMO+4 (0.47002), HOMO-2->LUMO+2 (0.41475), } \\
\text { HOMO-3->LUMO }(0.22461)\end{array}$ \\
\hline 509.06 & 0.0085 & HOMO->LUMO+5 (0.51760), HOMO-1->LUMO+4 (0.46873) \\
\hline 495.12 & 0.0401 & $\begin{array}{c}\text { HOMO-2->LUMO+3 (0.43612), HOMO-5->LUMO (-0.38731), } \\
\text { HOMO-6->LUMO }(-0.30885)\end{array}$ \\
\hline 487.83 & 0.0649 & $\begin{array}{c}\text { HOMO-5->LUMO (0.51683), HOMO-6->LUMO (-0.35046), } \\
\text { HOMO-3->LUMO+2 (0.22187) }\end{array}$ \\
\hline
\end{tabular}




\section{Copies of ${ }^{1} \mathrm{H},{ }^{13} \mathrm{C}$ NMR and Mass spectra of compounds}
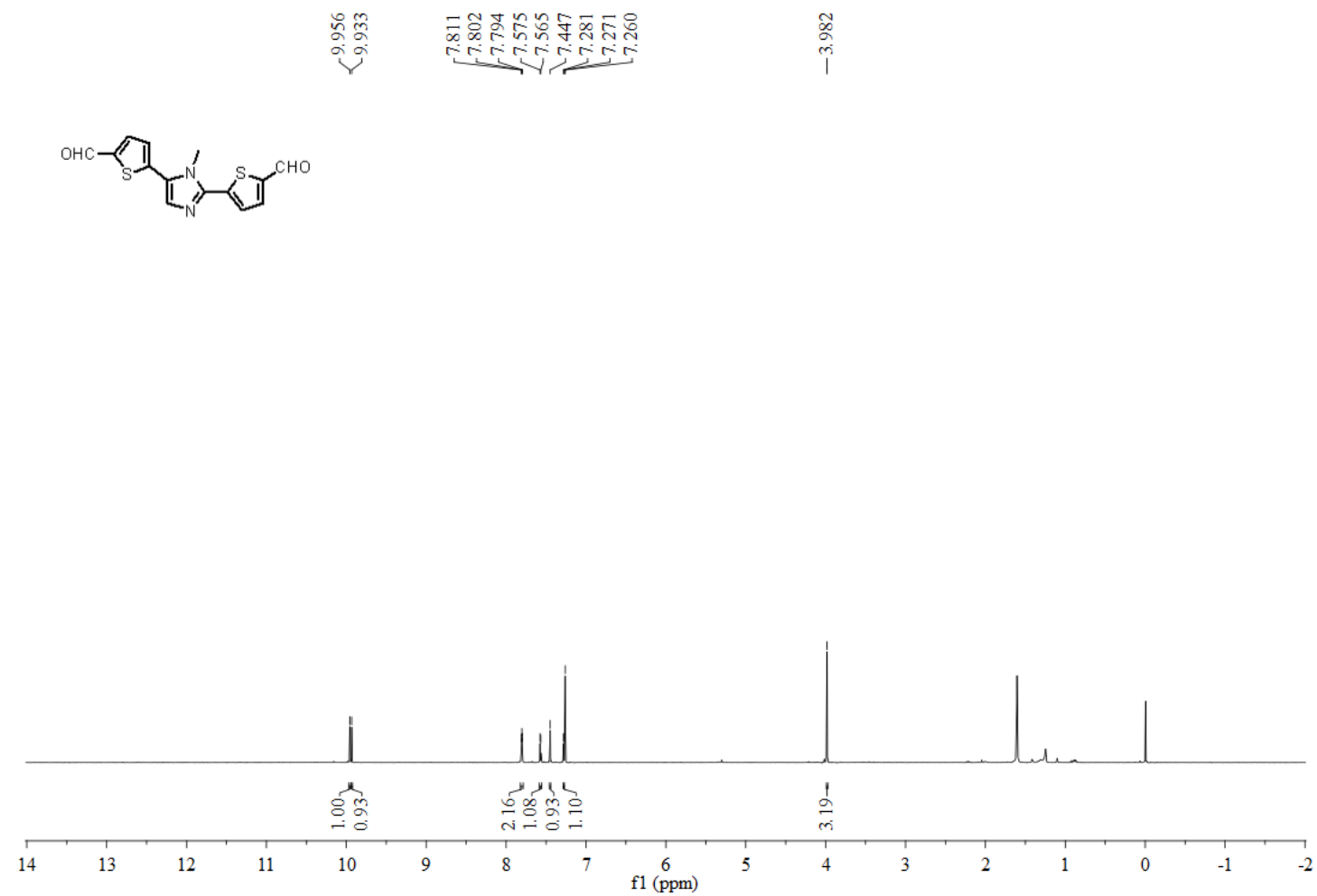

Figure S33. ${ }^{1} \mathrm{H}$ NMR spectrum of $1\left(400 \mathrm{MHz}, \mathrm{CDCl}_{3}\right)$.

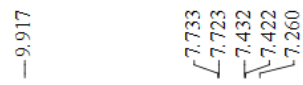

${ }^{\mathrm{OHC}} \mathrm{T}^{\mathrm{S}} \mathrm{J}-\mathrm{S}^{\mathrm{S}} \mathrm{T}^{\mathrm{CHO}}$

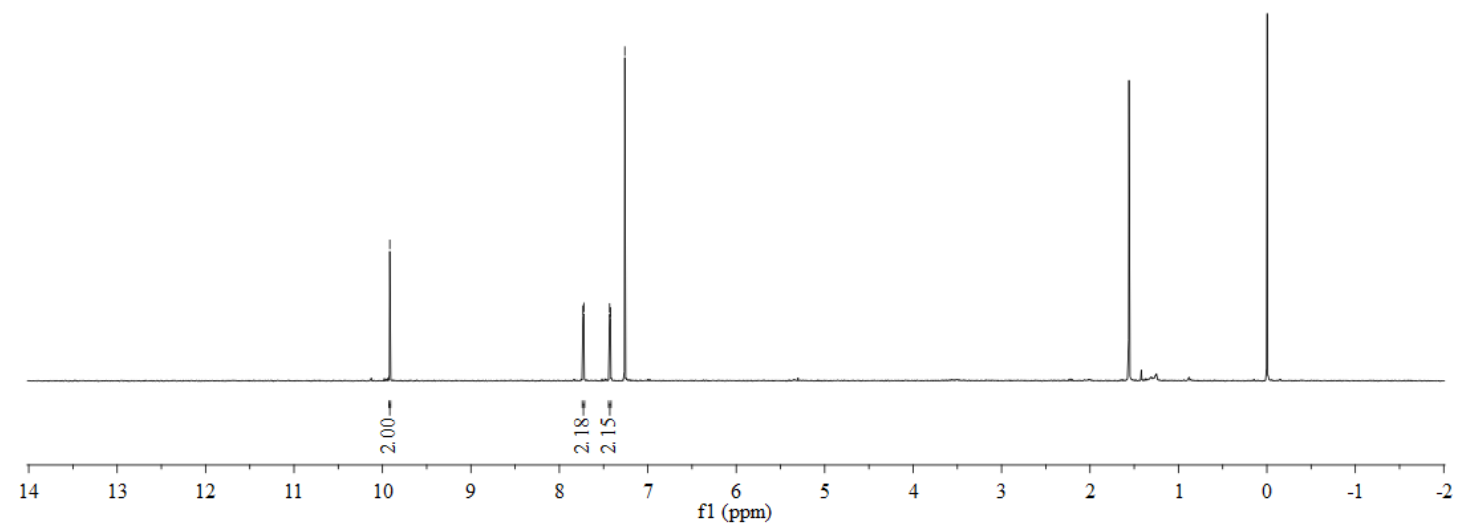

Figure S34. ${ }^{1} \mathrm{H}$ NMR spectrum of $2\left(400 \mathrm{MHz}, \mathrm{CDCl}_{3}\right)$. 

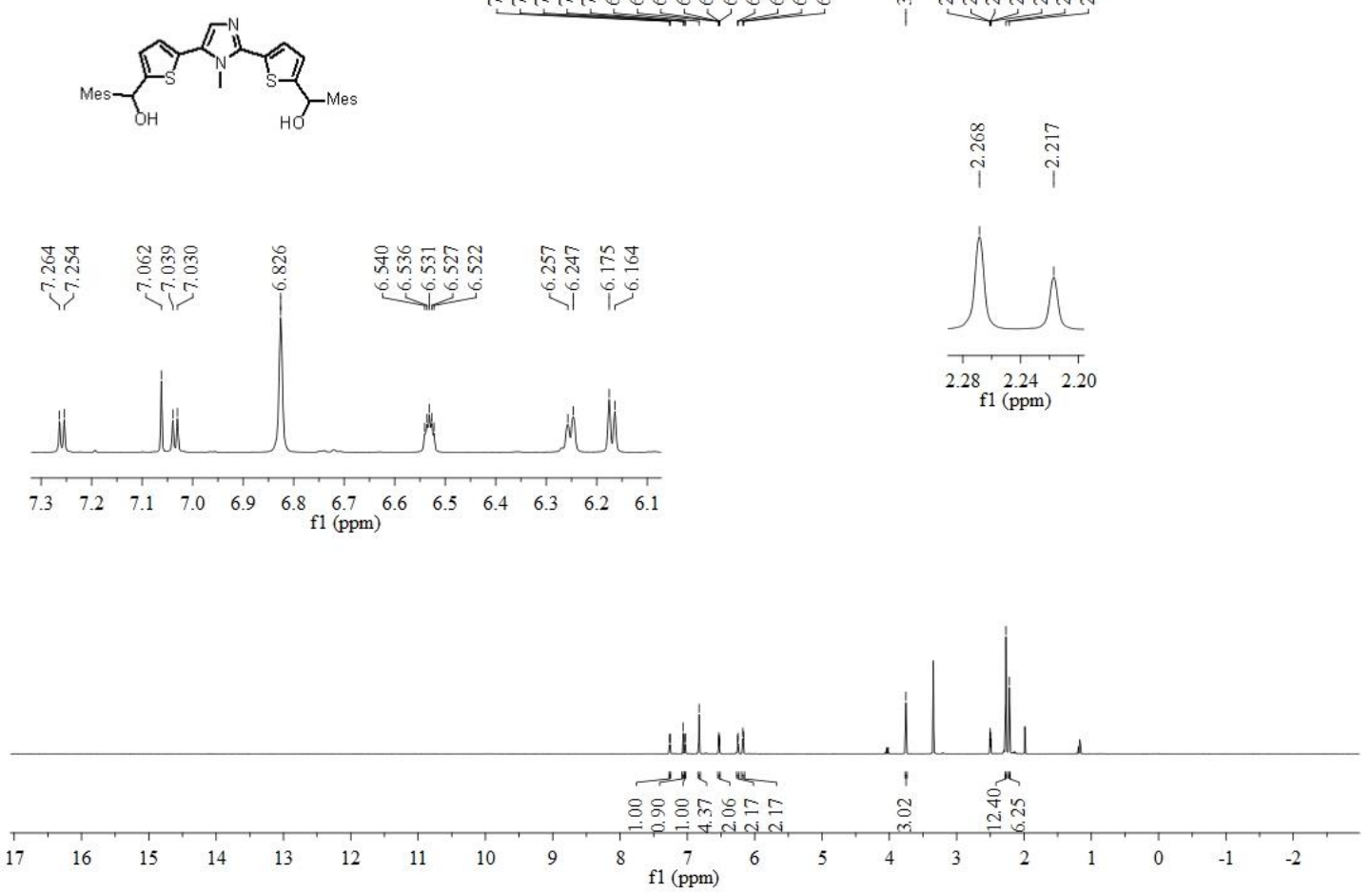

Figure S35. ${ }^{1} \mathrm{H}$ NMR spectrum of 3 (400 MHz, DMSO- $\left.d_{6}\right)$.

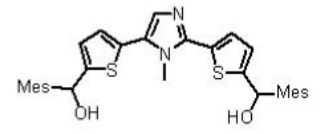
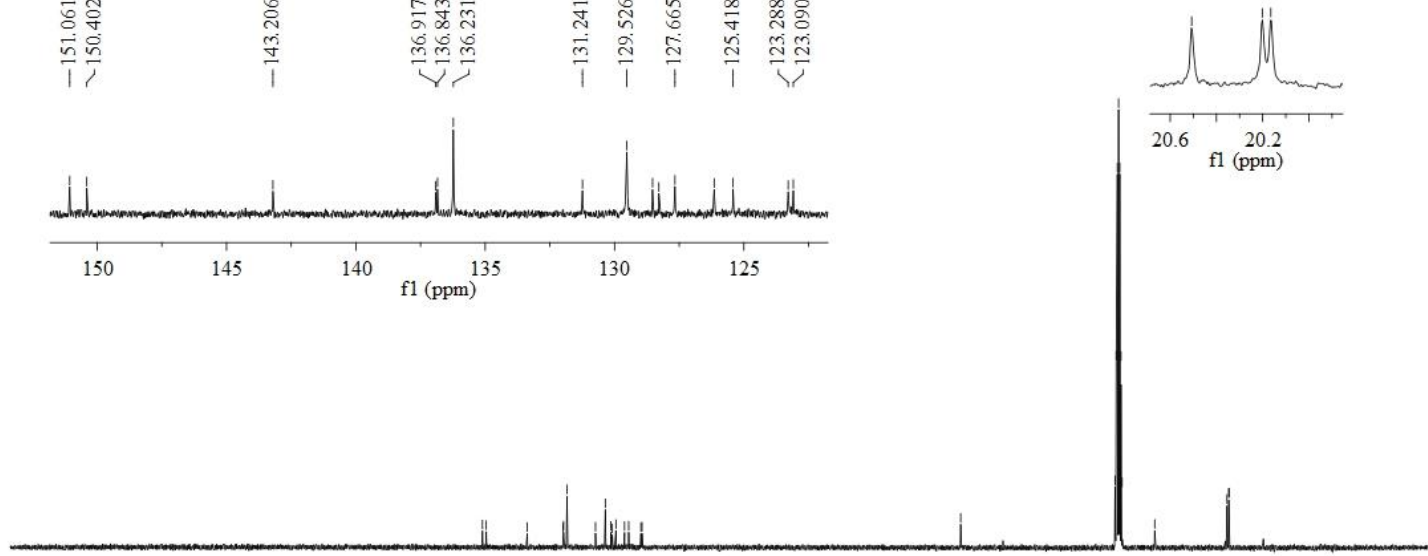

$\begin{array}{lllllllllllllllllllllllll}230 & 220 & 210 & 200 & 190 & 180 & 170 & 160 & 150 & 140 & 130 & 120 & 110 & 100 & 90 & 80 & 70 & 60 & 50 & 40 & 30 & 20 & 10 & 0 & -10\end{array}$

Figure S36. ${ }^{13} \mathrm{C}$ NMR spectrum of 3 (400 MHz, DMSO- $\left.d_{6}\right)$. 


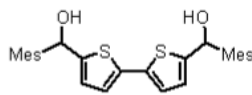

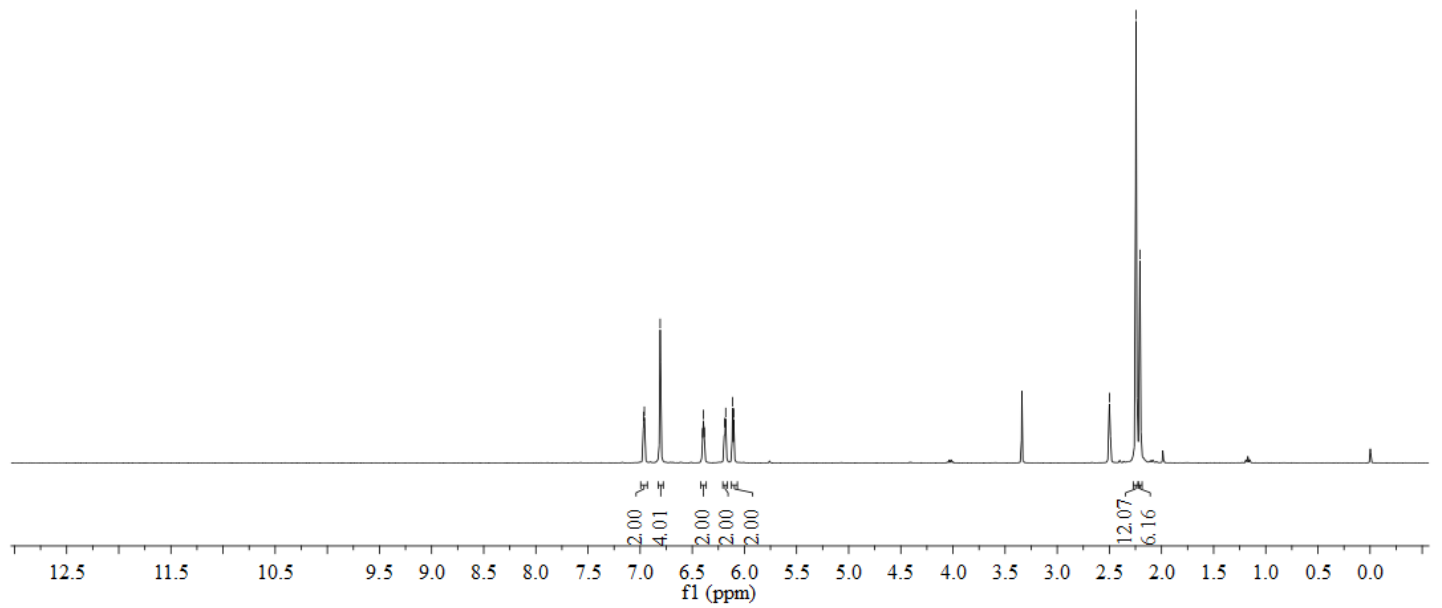

Figure S37. ${ }^{1} \mathrm{H}$ NMR spectrum of 4 (400 MHz, DMSO- $\left.d_{6}\right)$.

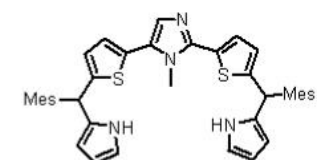

ஓ

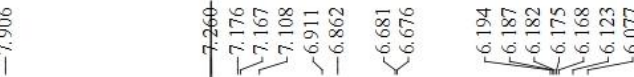

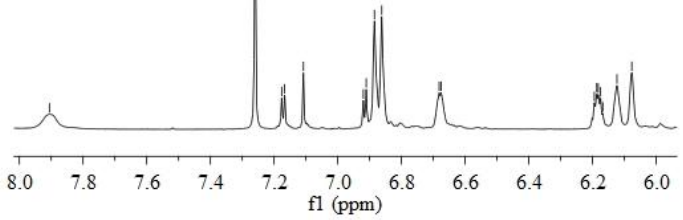

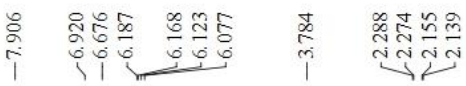
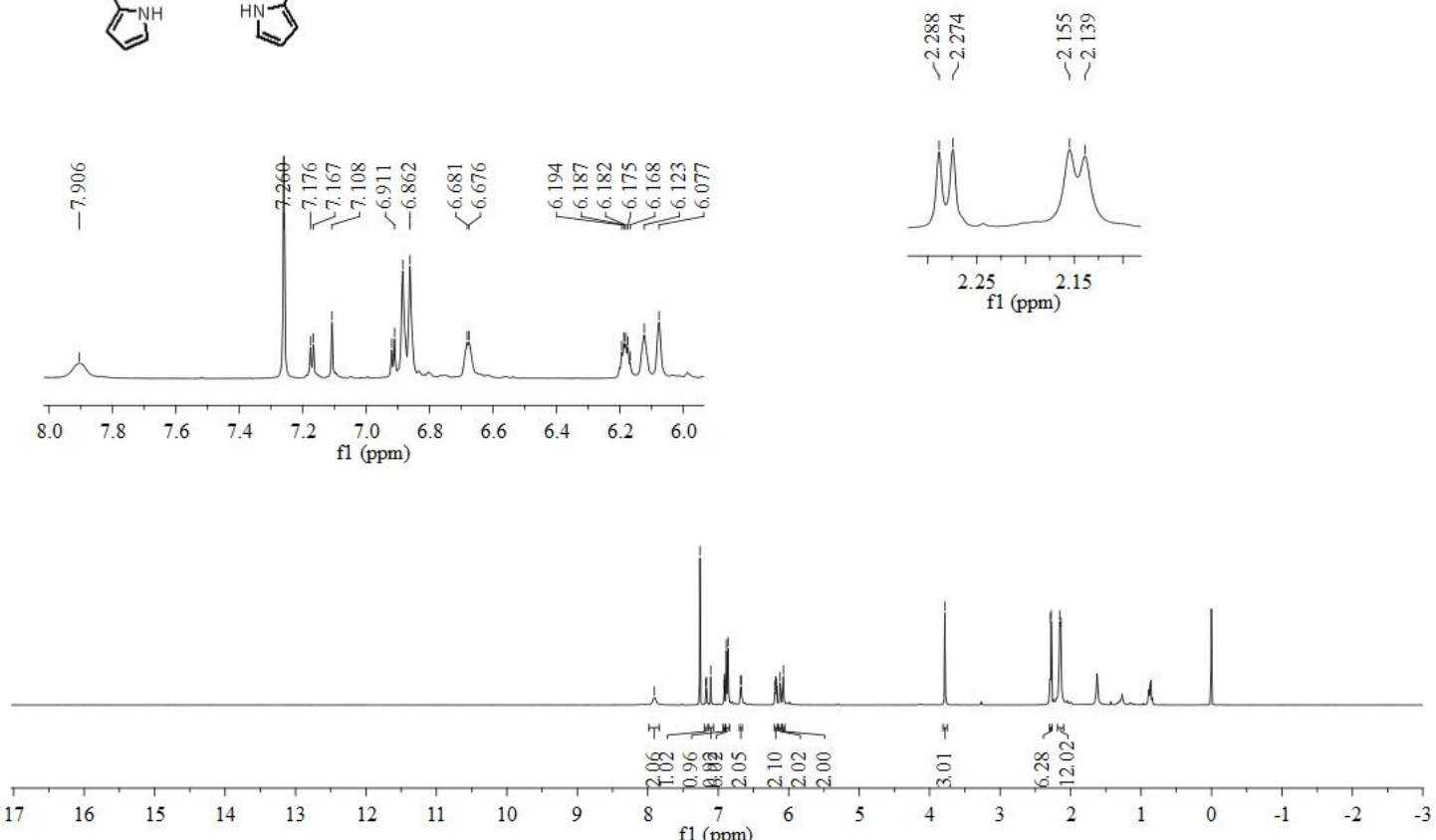

Figure S38. ${ }^{1} \mathrm{H}$ NMR spectrum of $5\left(400 \mathrm{MHz}, \mathrm{CDCl}_{3}\right)$. 

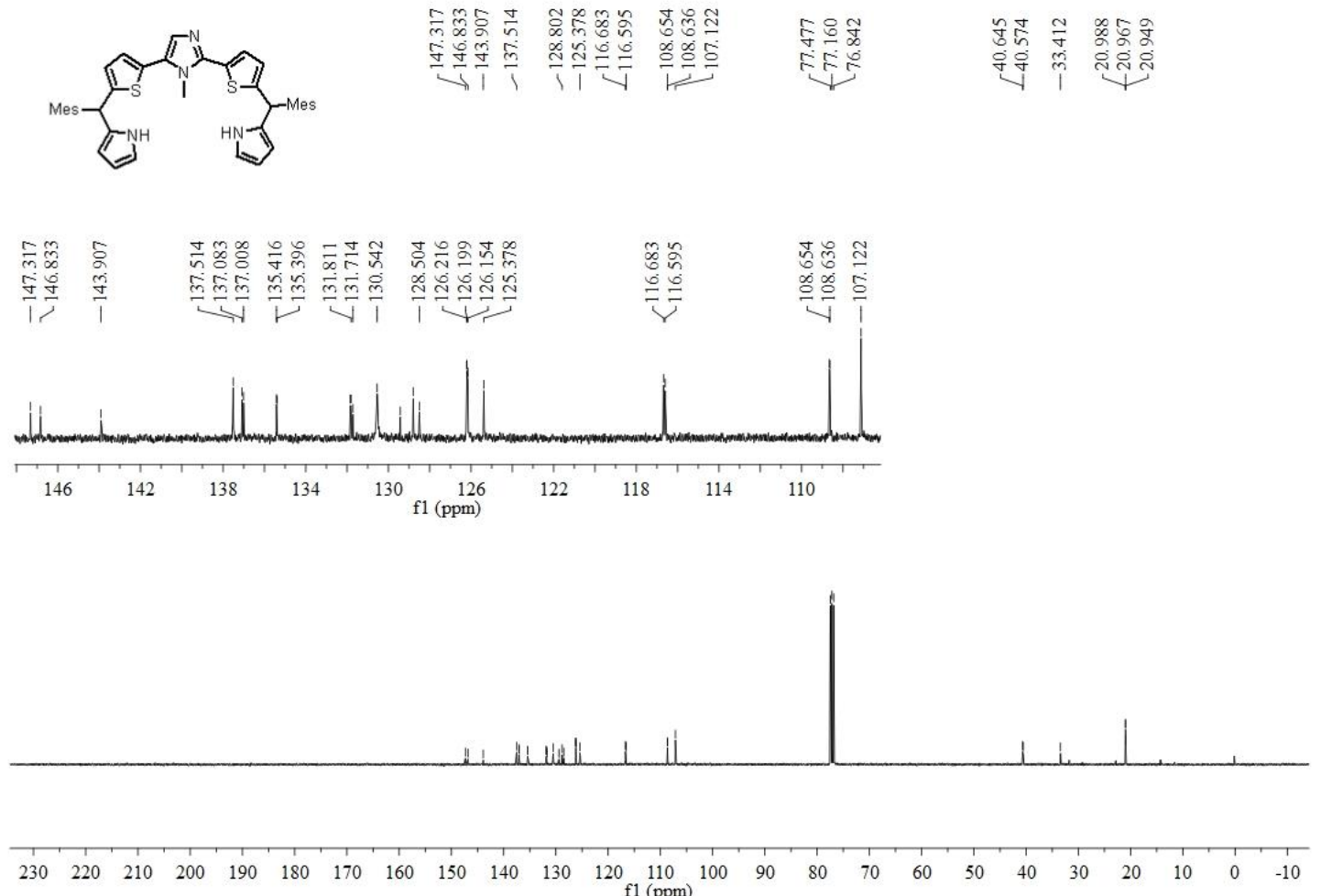

Figure S39. ${ }^{13} \mathrm{C} \mathrm{NMR}$ spectrum of $\mathbf{5}\left(400 \mathrm{MHz}, \mathrm{CDCl}_{3}\right)$.

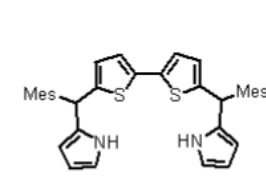

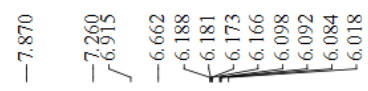

$\underset{\substack{\infty \\ N}}{\stackrel{m}{N}}$
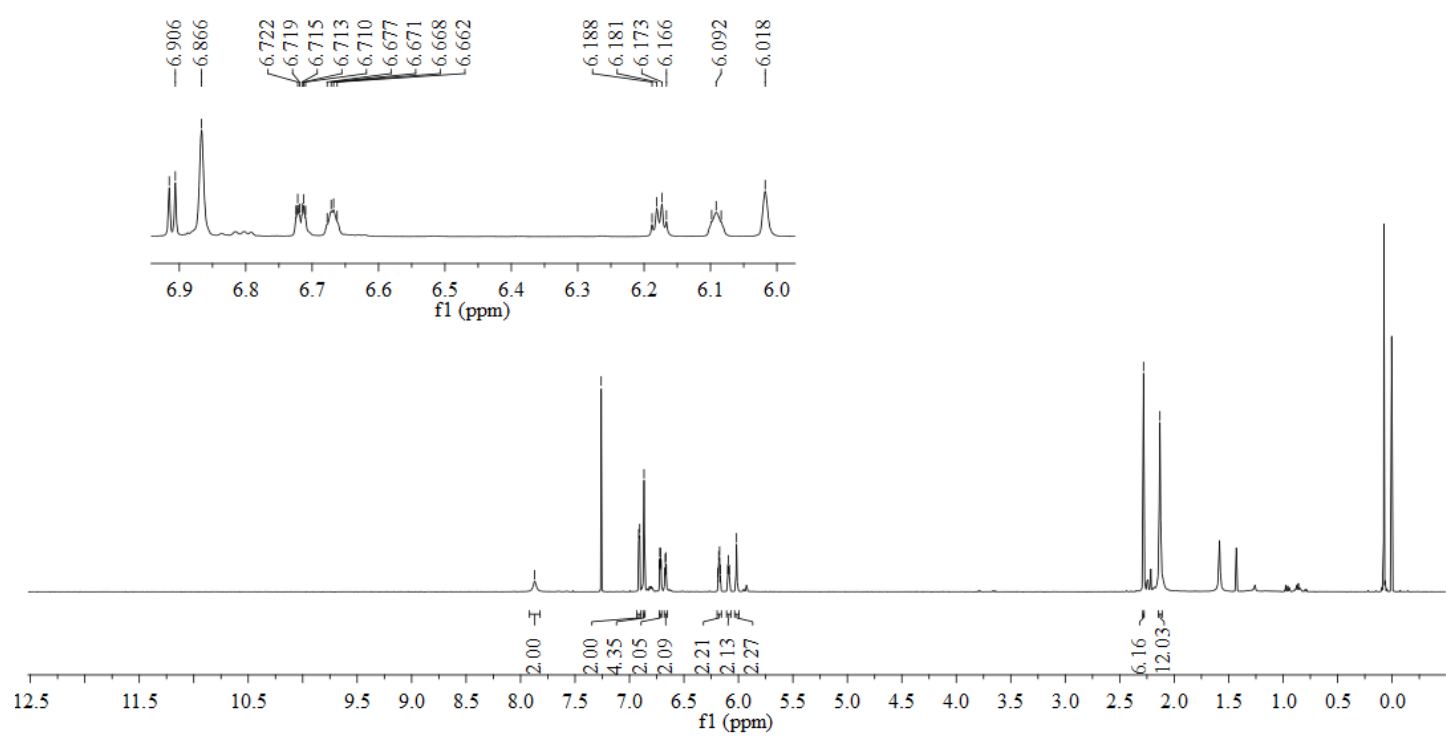

Figure S40. ${ }^{1} \mathrm{H}$ NMR spectrum of $6\left(400 \mathrm{MHz}, \mathrm{CDCl}_{3}\right)$. 


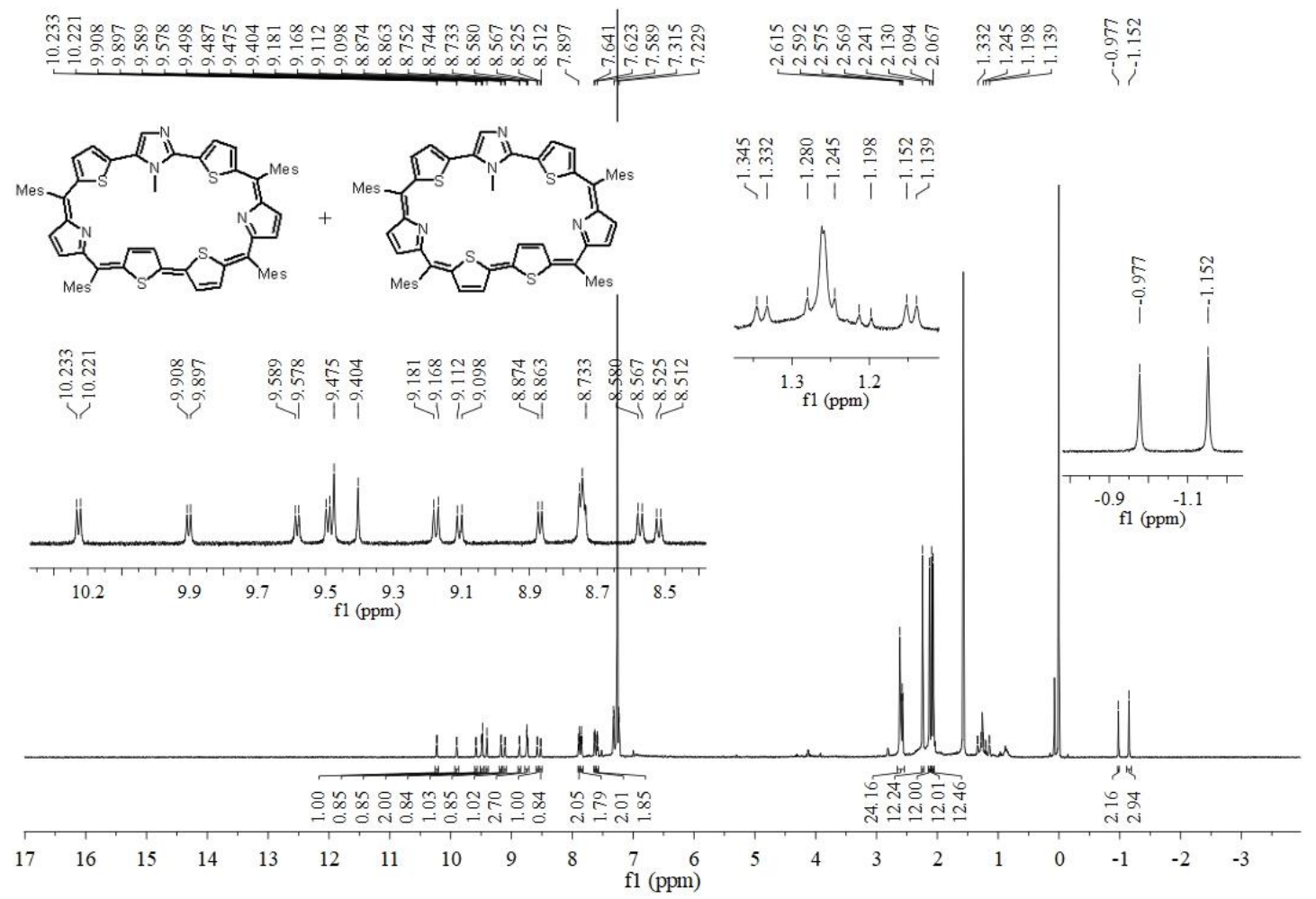

Figure S41. ${ }^{1} \mathrm{H}$ NMR spectrum of $7\left(400 \mathrm{MHz}, \mathrm{CDCl}_{3}\right)$.

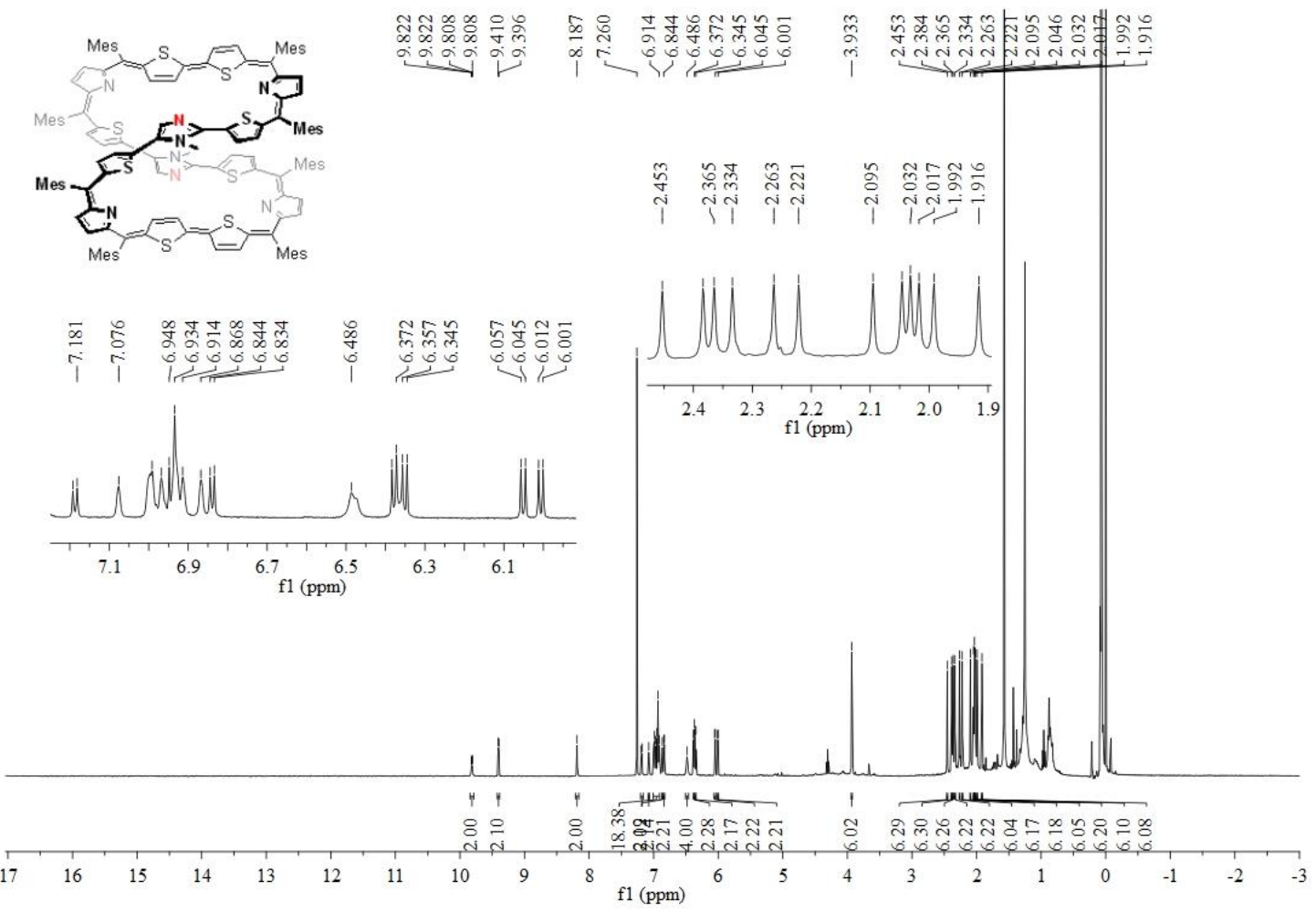

Figure S42. ${ }^{1} \mathrm{H}$ NMR spectrum of $8\left(400 \mathrm{MHz}, \mathrm{CDCl}_{3}\right)$. 


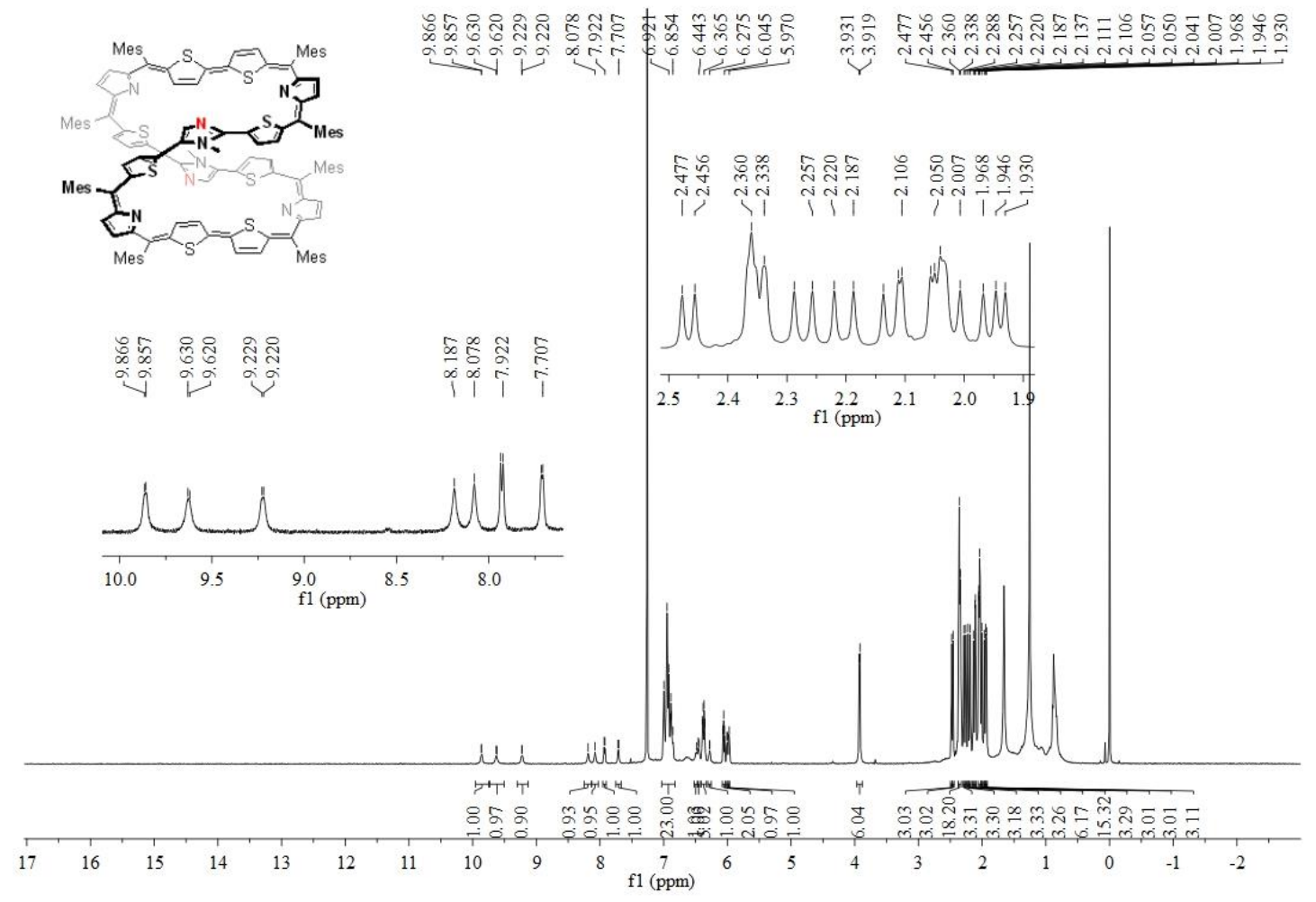

Figure S43. ${ }^{1} \mathrm{H}$ NMR spectrum of $9\left(400 \mathrm{MHz}, \mathrm{CDCl}_{3}\right)$.

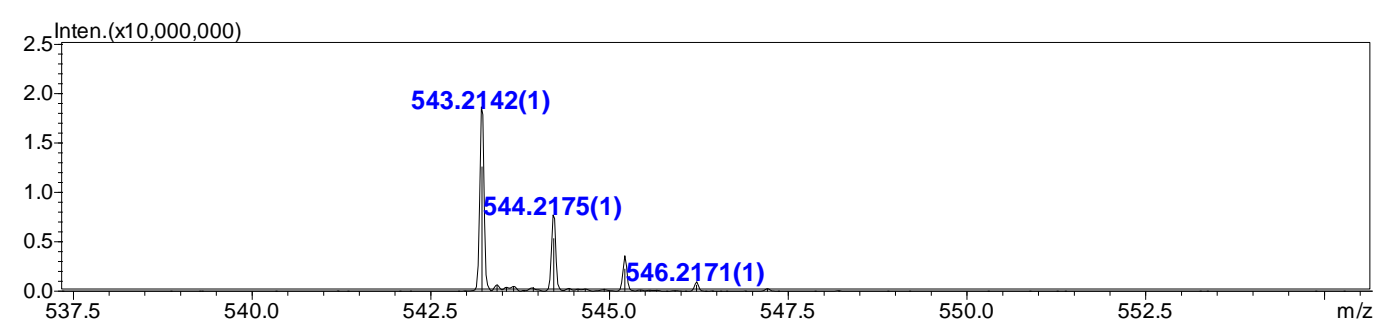

Figure S44. HR mass spectrum of 3.

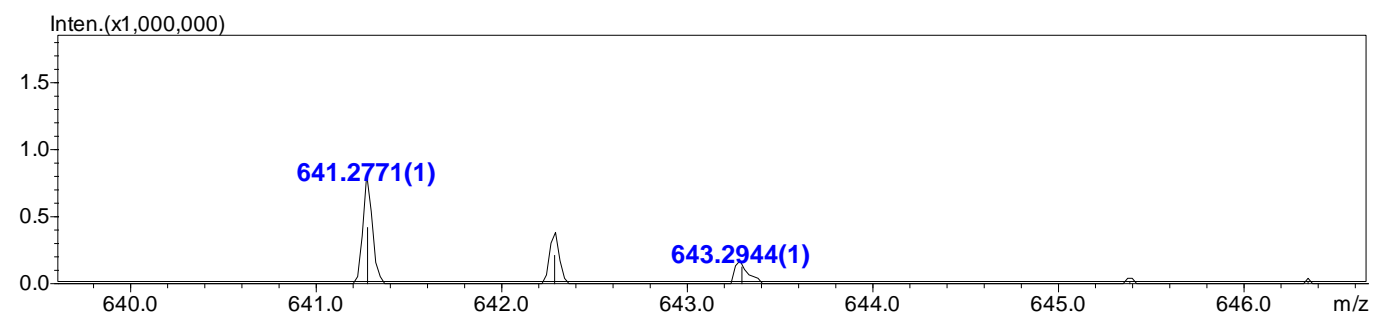

Figure S45. HR mass spectrum of 5. 


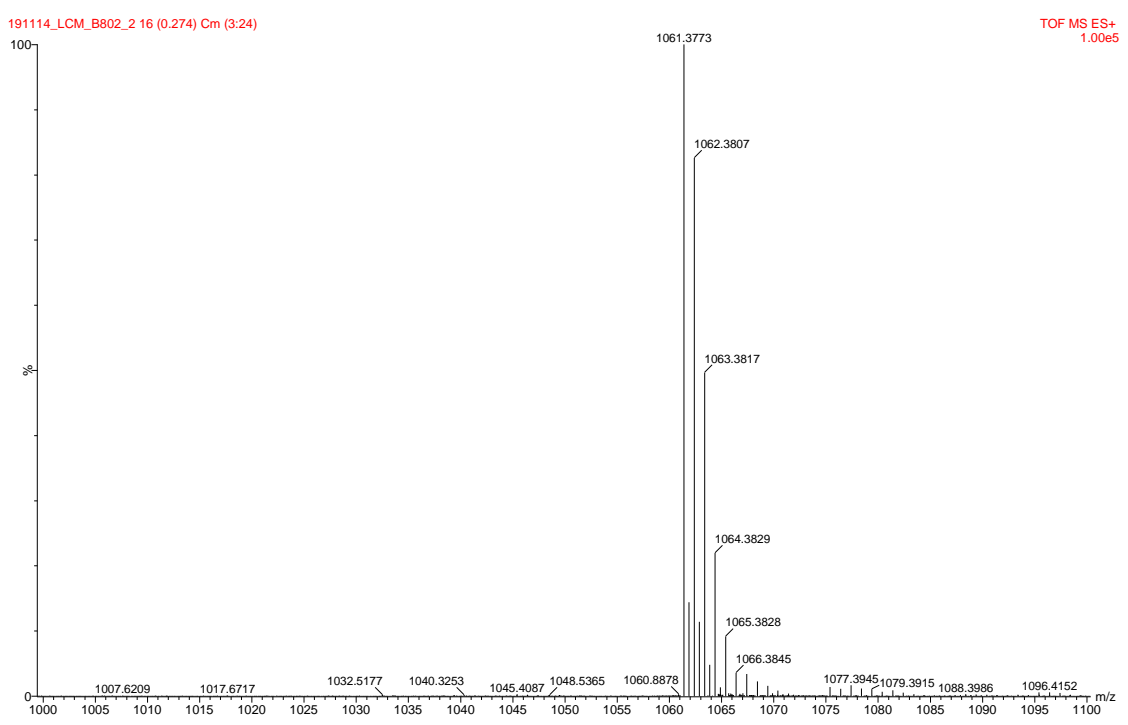

Figure S46. HR mass spectrum of the [30]heptaphyrin 7.

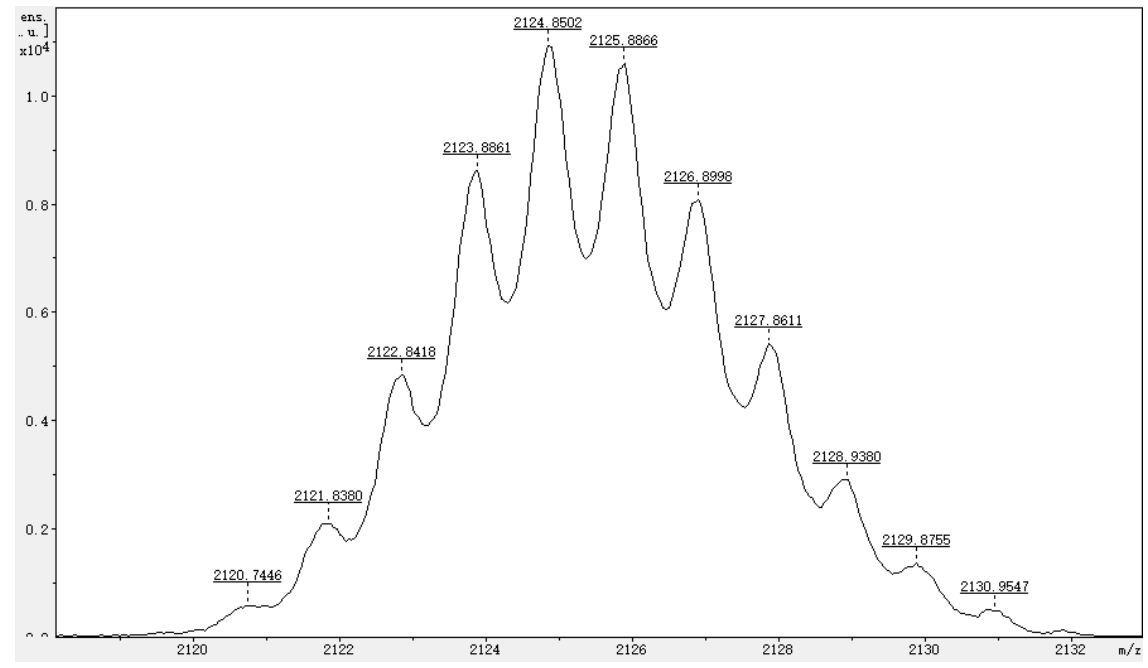

Figure S47. MALDI-TOF-MS of the [60]tetradecaphyrin 8.

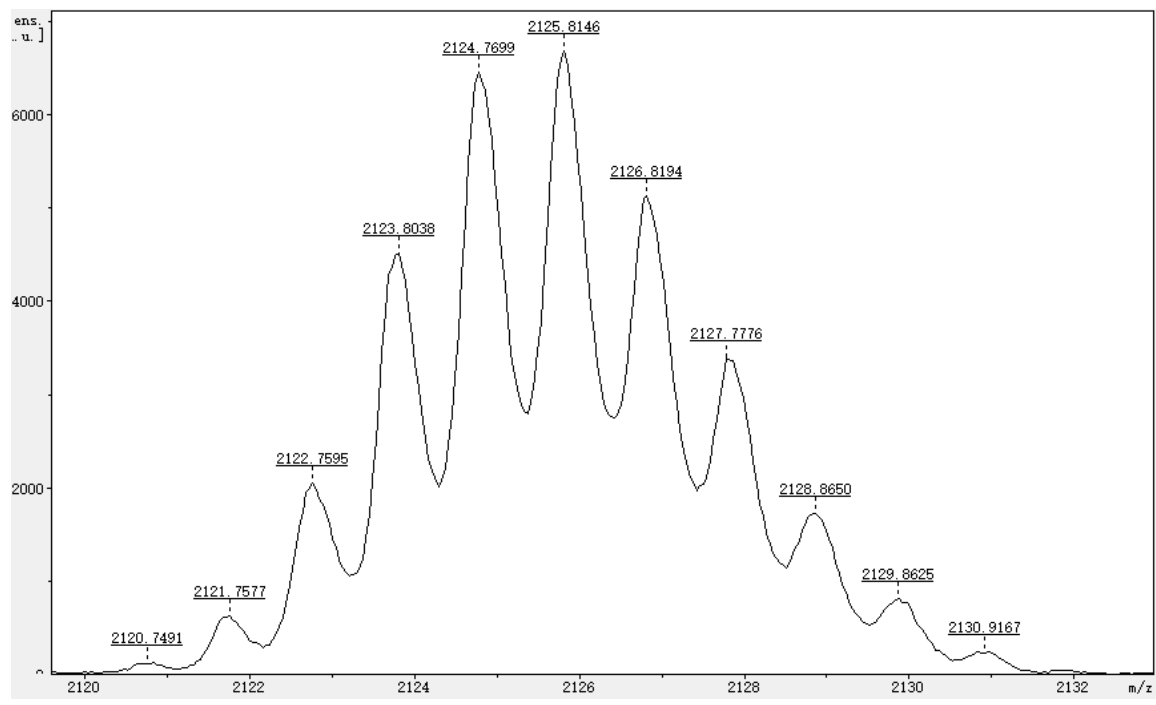

Figure S48. MALDI-TOF-MS of the [60]tetradecaphyrin 9. 


\section{References}

1. Xi, P.; Yang, F.; Qin, S.; Zhao, D.; Lan, J.; Gao, G.; Hu, C.; You, J. Palladium(II)-Catalyzed Oxidative C-H/C-H Cross-Coupling of Heteroarenes. J. Am. Chem. Soc. 2010, 132, 1822.

2. Gokulnath, S.; Prabhuraja, V.; Chandrashekar, T. K.; Suresh, C. H. Modified 26 and $28 \pi$ Hexaphyrins with five meso-Links: Optical, Redox and Structural Properties. Chem. Asian J. 2009, 4,861 .

3. Adinarayana, B.; Thomas, A. P.; Suresh, C. H.; Srinivasan, A. A 6,11,16-Triarylbiphenylcorrole with an adj-CCNN Core: Stabilization of an Organocopper(III) Complex. Angew. Chem. Int. Ed. 2015, 54, 10478.

4. Anand, V. R. G.; Pushpan, S. K.; Srinivasan, A.; Narayanan, S. J.; Sridevi, B.; Chandrashekar, T. K.; Roy, R.; Joshi, B. S. Meso Aryl Heptaphyrins: The First 30 $\pi$ Aromatic Expanded Porphyrins with an Inverted Structure. Org. Lett. 2000, 2, 3829.

5. Frisch, M. J.; Trucks, G. W.; Schlegel, H. B.; Scuseria, G. E.; Robb, M. A.; Cheeseman, J. R.; Scalmani, G.; Barone, V.; Mennucci, B.; Petersson, G. A.; Nakatsuji, H.; Caricato, M.; Li, X.; Hratchian, H. P.; Izmaylov, A. F.; Bloino, J.; Zheng, G.; Sonnenberg, J. L.; Hada, M.; Ehara, M.; Toyota, K.; Fukuda, R.; Hasegawa, J.; Ishida, M.; Nakajima, T.; Honda, Y.; Kitao, O.; Nakai, H.; Vreven, T.; Jr. Montgomery, J. A.; Peralta, J. E.; Ogliaro, F.; Bearpark, M.; Heyd, J. J.; Brothers, E.; Kudin, K. N.; Staroverov, V. N.; Kobayashi, R.; Normand, J.; Raghavachari, K.; Rendell, A.; Burant, J. C.; Iyengar, S. S.; Tomasi, J.; Cossi, M.; Rega, N.; Millam, N. J.; Klene, M.; Knox, J. E.; Cross, J. B.; Bakken, V.; Adamo, C.; Jaramillo, J.; Gomperts, R.; Stratmann, R. E.; Yazyev, O.; Austin, A. J.; Cammi, R.; Pomelli, C.; Ochterski, J. W.; Martin, R. L.; Morokuma, K.; Zakrzewski, V. G.; Voth, G. A.; Salvador, P.; Dannenberg, J. J.; Dapprich, S.; Daniels, A. D.; Farkas, Ö.; Foresman, J. B.; Ortiz, J. V.; Cioslowski, J.; Fox, D. J. Gaussian 09, Revision D.01, Gaussian, Inc., Wallingford CT, 2009.

6. (a) Becke, A. D. Density-Functional Thermochemistry. III. The Role of Exact Exchange. $J$. Chem. Phys. 1993, 98, 5648. (b) Lee, C.; Yang, W.; Parr, R. G. Development of the Colic-Salvetti Correlation-Energy Formula into A Functional of the Electron Density. Phys. Rev. B. 1988, 37, 785. 


\section{Appendix: Cartesian coordinates of the optimized structures}

7a (Sum of imaginary frequencies $=0$, total energy $=-4439.23046451$ Hartrees)

\begin{tabular}{|c|c|c|c|c|c|}
\hline \multirow{2}{*}{$\begin{array}{l}\text { Center } \\
\text { Number }\end{array}$} & \multirow{2}{*}{$\begin{array}{l}\text { Atomic } \\
\text { Number }\end{array}$} & \multirow{2}{*}{$\begin{array}{l}\text { Atomic } \\
\text { Type }\end{array}$} & \multicolumn{3}{|c|}{ Coordinates (Angstroms) } \\
\hline & & & $X$ & $\mathrm{Y}$ & Z \\
\hline 1 & 6 & 0 & -1.638642 & -4.214559 & 0.135315 \\
\hline 2 & 6 & 0 & 1.987483 & -3.946695 & -0.009117 \\
\hline 3 & 6 & 0 & -5.252407 & -2.636459 & 0.168613 \\
\hline 4 & 6 & 0 & -6.355654 & -1.744387 & 0.077313 \\
\hline 5 & 6 & 0 & -6.217613 & -0.366256 & -0.115815 \\
\hline 6 & 6 & 0 & 5.806254 & -1.005099 & -0.022859 \\
\hline 7 & 6 & 0 & -7.291846 & 0.619834 & -0.158591 \\
\hline 8 & 6 & 0 & -6.685190 & 1.829987 & -0.289176 \\
\hline 9 & 6 & 0 & -5.245642 & 1.566362 & -0.342063 \\
\hline 10 & 7 & 0 & -5.000359 & 0.244840 & -0.241992 \\
\hline 11 & 6 & 0 & -4.240624 & 2.580517 & -0.435839 \\
\hline 12 & 7 & 0 & -0.485953 & -3.457181 & 0.331651 \\
\hline 13 & 6 & 0 & 0.587332 & -4.260226 & -0.006130 \\
\hline 14 & 7 & 0 & 0.167021 & -5.484953 & -0.382917 \\
\hline 15 & 6 & 0 & -1.167365 & -5.463455 & -0.295618 \\
\hline 16 & 16 & 0 & -3.558344 & -2.138174 & 0.100663 \\
\hline 17 & 6 & 0 & -3.001208 & -3.800261 & 0.249263 \\
\hline 18 & 6 & 0 & -4.089081 & -4.673744 & 0.354850 \\
\hline 19 & 6 & 0 & -5.324775 & -4.031712 & 0.312850 \\
\hline 20 & 16 & 0 & 2.759192 & -2.363377 & 0.053959 \\
\hline 21 & 6 & 0 & 4.368276 & -3.083875 & -0.097092 \\
\hline 22 & 6 & 0 & 4.246983 & -4.480754 & -0.205270 \\
\hline 23 & 6 & 0 & 2.943570 & -4.953664 & -0.162916 \\
\hline 24 & 6 & 0 & 5.608111 & -2.388865 & -0.086008 \\
\hline 25 & 7 & 0 & 4.828673 & -0.038991 & -0.021341 \\
\hline 26 & 6 & 0 & 5.484161 & 1.139357 & 0.075979 \\
\hline 27 & 6 & 0 & 6.931492 & 0.940981 & 0.147054 \\
\hline 28 & 6 & 0 & 7.132503 & -0.396401 & 0.072402 \\
\hline 29 & 6 & 0 & -0.421278 & -2.171629 & 1.020946 \\
\hline 30 & 6 & 0 & 4.880035 & 2.428891 & 0.095025 \\
\hline 31 & 6 & 0 & -0.337964 & 2.373994 & -0.612308 \\
\hline 32 & 6 & 0 & -0.885066 & 1.111135 & -0.952784 \\
\hline 33 & 6 & 0 & -2.258099 & 1.060523 & -0.942197 \\
\hline 34 & 6 & 0 & -2.886094 & 2.292299 & -0.603338 \\
\hline 35 & 16 & 0 & -1.643828 & 3.539782 & -0.354766 \\
\hline 36 & 6 & 0 & 1.005888 & 2.696510 & -0.429701 \\
\hline 37 & 6 & 0 & 1.557323 & 3.981596 & -0.202077 \\
\hline
\end{tabular}




\begin{tabular}{|c|c|c|c|c|c|}
\hline 38 & 6 & 0 & 2.916590 & 3.975472 & -0.016119 \\
\hline 39 & 6 & 0 & 3.519194 & 2.682491 & -0.068735 \\
\hline 40 & 16 & 0 & 2.278326 & 1.459302 & -0.375575 \\
\hline 41 & 6 & 0 & -7.734139 & -2.332349 & 0.190167 \\
\hline 42 & 6 & 0 & -8.436609 & -2.709921 & -0.973324 \\
\hline 43 & 6 & 0 & -9.721096 & -3.249362 & -0.844222 \\
\hline 44 & 6 & 0 & -10.327947 & -3.425866 & 0.401765 \\
\hline 45 & 6 & 0 & -9.614843 & -3.039811 & 1.540249 \\
\hline 46 & 6 & 0 & -8.328268 & -2.497704 & 1.459599 \\
\hline 47 & 6 & 0 & -7.604541 & -2.084986 & 2.721216 \\
\hline 48 & 6 & 0 & -7.829486 & -2.533135 & -2.346725 \\
\hline 49 & 6 & 0 & -11.703398 & -4.039276 & 0.518291 \\
\hline 50 & 6 & 0 & 6.839713 & -3.258087 & -0.147640 \\
\hline 51 & 6 & 0 & 7.431094 & -3.551332 & -1.394358 \\
\hline 52 & 6 & 0 & 8.570368 & -4.362484 & -1.430093 \\
\hline 53 & 6 & 0 & 9.142564 & -4.886816 & -0.268080 \\
\hline 54 & 6 & 0 & 8.538203 & -4.584297 & 0.955067 \\
\hline 55 & 6 & 0 & 7.396690 & -3.780115 & 1.038761 \\
\hline 56 & 6 & 0 & 6.786132 & -3.480021 & 2.388778 \\
\hline 57 & 6 & 0 & 6.858232 & -3.003639 & -2.681939 \\
\hline 58 & 6 & 0 & 10.392404 & -5.732838 & -0.329633 \\
\hline 59 & 6 & 0 & 5.781932 & 3.616390 & 0.309530 \\
\hline 60 & 6 & 0 & 6.085631 & 4.043585 & 1.619594 \\
\hline 61 & 6 & 0 & 6.926673 & 5.147245 & 1.796682 \\
\hline 62 & 6 & 0 & 7.480314 & 5.836445 & 0.714294 \\
\hline 63 & 6 & 0 & 7.165161 & 5.397107 & -0.574210 \\
\hline 64 & 6 & 0 & 6.326831 & 4.299623 & -0.798191 \\
\hline 65 & 6 & 0 & 6.023040 & 3.862289 & -2.213323 \\
\hline 66 & 6 & 0 & 5.516968 & 3.329967 & 2.825023 \\
\hline 67 & 6 & 0 & 8.414837 & 7.003124 & 0.931372 \\
\hline 68 & 6 & 0 & -4.661006 & 4.014515 & -0.282265 \\
\hline 69 & 6 & 0 & -4.855725 & 4.817622 & -1.427278 \\
\hline 70 & 6 & 0 & -5.248396 & 6.149624 & -1.262871 \\
\hline 71 & 6 & 0 & -5.446209 & 6.711840 & 0.000960 \\
\hline 72 & 6 & 0 & -5.253143 & 5.897867 & 1.120263 \\
\hline 73 & 6 & 0 & -4.867579 & 4.558760 & 1.004304 \\
\hline 74 & 6 & 0 & -4.686536 & 3.718868 & 2.248290 \\
\hline 75 & 6 & 0 & -4.662411 & 4.257515 & -2.818159 \\
\hline 76 & 6 & 0 & -5.833341 & 8.163728 & 0.153826 \\
\hline 77 & 1 & 0 & -8.349441 & 0.407396 & -0.084018 \\
\hline 78 & 1 & 0 & -7.148402 & 2.804913 & -0.352773 \\
\hline 79 & 1 & 0 & -1.771850 & -6.311502 & -0.588071 \\
\hline 80 & 1 & 0 & -3.972475 & -5.744814 & 0.471254 \\
\hline 81 & 1 & 0 & -6.275396 & -4.545354 & 0.385815 \\
\hline
\end{tabular}




\begin{tabular}{|c|c|c|c|c|c|}
\hline 82 & 1 & 0 & 5.115381 & -5.117761 & -0.308565 \\
\hline 83 & 1 & 0 & 2.654932 & -5.993189 & -0.241258 \\
\hline 84 & 1 & 0 & 7.671007 & 1.724339 & 0.231346 \\
\hline 85 & 1 & 0 & 8.070717 & -0.931826 & 0.090113 \\
\hline 86 & 1 & 0 & -1.217622 & -2.123436 & 1.764873 \\
\hline 87 & 1 & 0 & 0.538201 & -2.084965 & 1.530859 \\
\hline 88 & 1 & 0 & -0.535692 & -1.330503 & 0.333924 \\
\hline 89 & 1 & 0 & -0.250759 & 0.275263 & -1.227139 \\
\hline 90 & 1 & 0 & -2.851113 & 0.188436 & -1.172288 \\
\hline 91 & 1 & 0 & 0.949299 & 4.879439 & -0.193817 \\
\hline 92 & 1 & 0 & 3.511002 & 4.863090 & 0.158505 \\
\hline 93 & 1 & 0 & -10.260809 & -3.537025 & -1.743797 \\
\hline 94 & 1 & 0 & -10.071779 & -3.159630 & 2.520191 \\
\hline 95 & 1 & 0 & -8.213029 & -2.293247 & 3.605007 \\
\hline 96 & 1 & 0 & -6.653118 & -2.615783 & 2.831775 \\
\hline 97 & 1 & 0 & -7.369963 & -1.015225 & 2.714952 \\
\hline 98 & 1 & 0 & -6.861206 & -3.037999 & -2.426650 \\
\hline 99 & 1 & 0 & -8.488410 & -2.939149 & -3.118556 \\
\hline 100 & 1 & 0 & -7.653760 & -1.476119 & -2.573314 \\
\hline 101 & 1 & 0 & -12.293276 & -3.874388 & -0.388072 \\
\hline 102 & 1 & 0 & -11.642288 & -5.123667 & 0.674448 \\
\hline 103 & 1 & 0 & -12.257546 & -3.621918 & 1.364549 \\
\hline 104 & 1 & 0 & 9.020423 & -4.590114 & -2.393948 \\
\hline 105 & 1 & 0 & 8.964753 & -4.984580 & 1.872266 \\
\hline 106 & 1 & 0 & 7.364914 & -3.946715 & 3.190011 \\
\hline 107 & 1 & 0 & 6.748755 & -2.402405 & 2.579543 \\
\hline 108 & 1 & 0 & 5.757128 & -3.849081 & 2.455925 \\
\hline 109 & 1 & 0 & 6.922888 & -1.910741 & -2.714052 \\
\hline 110 & 1 & 0 & 7.397549 & -3.399243 & -3.546506 \\
\hline 111 & 1 & 0 & 5.800002 & -3.260515 & -2.794916 \\
\hline 112 & 1 & 0 & 10.449420 & -6.295916 & -1.266023 \\
\hline 113 & 1 & 0 & 11.294989 & -5.111787 & -0.268995 \\
\hline 114 & 1 & 0 & 10.433942 & -6.446392 & 0.498704 \\
\hline 115 & 1 & 0 & 7.154355 & 5.475277 & 2.808628 \\
\hline 116 & 1 & 0 & 7.582718 & 5.920891 & -1.431383 \\
\hline 117 & 1 & 0 & 6.559971 & 4.482165 & -2.936096 \\
\hline 118 & 1 & 0 & 6.311594 & 2.819098 & -2.379594 \\
\hline 119 & 1 & 0 & 4.953155 & 3.932516 & -2.436789 \\
\hline 120 & 1 & 0 & 5.832902 & 2.281692 & 2.854449 \\
\hline 121 & 1 & 0 & 5.844957 & 3.808414 & 3.751567 \\
\hline 122 & 1 & 0 & 4.421928 & 3.328131 & 2.813183 \\
\hline 123 & 1 & 0 & 8.172727 & 7.545301 & 1.850472 \\
\hline 124 & 1 & 0 & 9.455614 & 6.666499 & 1.018251 \\
\hline 125 & 1 & 0 & 8.372114 & 7.711242 & 0.098393 \\
\hline
\end{tabular}




$\begin{array}{llllll}126 & 1 & 0 & -5.405853 & 6.762422 & -2.147777 \\ 127 & 1 & 0 & -5.408633 & 6.313754 & 2.113358 \\ 128 & 1 & 0 & -4.788559 & 4.331110 & 3.148164 \\ 129 & 1 & 0 & -5.430419 & 2.916266 & 2.299912 \\ 130 & 1 & 0 & -3.701871 & 3.241367 & 2.272511 \\ 131 & 1 & 0 & -3.619215 & 3.974350 & -2.995761 \\ 132 & 1 & 0 & -5.267043 & 3.358554 & -2.977027 \\ 133 & 1 & 0 & -4.943143 & 4.993807 & -3.575799 \\ 134 & 1 & 0 & -6.394824 & 8.521929 & -0.714143 \\ 135 & 1 & 0 & -6.447532 & 8.323258 & 1.045260 \\ 136 & 1 & 0 & -4.946076 & 8.801661 & 0.253131\end{array}$

7b (Sum of imaginary frequencies $=0$, total energy $=-4439.22786707$ Hartrees $)$

\begin{tabular}{|c|c|c|c|c|c|}
\hline \multirow{2}{*}{$\begin{array}{l}\text { Center } \\
\text { Number }\end{array}$} & \multirow{2}{*}{$\begin{array}{l}\text { Atomic } \\
\text { Number }\end{array}$} & \multirow{2}{*}{$\begin{array}{l}\text { Atomic } \\
\text { Type }\end{array}$} & \multicolumn{3}{|c|}{ Coordinates (Angstroms) } \\
\hline & & & $\mathrm{X}$ & $\mathrm{Y}$ & $\mathrm{Z}$ \\
\hline 1 & 6 & 0 & 1.570928 & -4.178667 & 0.456036 \\
\hline 2 & 6 & 0 & -2.052380 & -3.965343 & 0.230571 \\
\hline 3 & 6 & 0 & 5.207292 & -2.658584 & 0.148654 \\
\hline 4 & 6 & 0 & 6.322525 & -1.790935 & -0.014177 \\
\hline 5 & 6 & 0 & 6.208991 & -0.410939 & -0.215522 \\
\hline 6 & 6 & 0 & -5.820594 & -0.981050 & -0.122580 \\
\hline 7 & 6 & 0 & 7.305854 & 0.548196 & -0.298986 \\
\hline 8 & 6 & 0 & 6.726122 & 1.775293 & -0.387555 \\
\hline 9 & 6 & 0 & 5.279420 & 1.549463 & -0.382144 \\
\hline 10 & 7 & 0 & 5.003870 & 0.233233 & -0.285348 \\
\hline 11 & 6 & 0 & 4.296426 & 2.590083 & -0.415644 \\
\hline 12 & 7 & 0 & 0.436422 & -3.435864 & 0.180568 \\
\hline 13 & 6 & 0 & -0.661039 & -4.257630 & 0.396643 \\
\hline 14 & 6 & 0 & -0.098587 & -5.481985 & 0.801173 \\
\hline 15 & 7 & 0 & 1.237235 & -5.428433 & 0.834242 \\
\hline 16 & 16 & 0 & 3.524206 & -2.128149 & 0.104433 \\
\hline 17 & 6 & 0 & 2.940686 & -3.767119 & 0.362293 \\
\hline 18 & 6 & 0 & 4.011221 & -4.655798 & 0.501175 \\
\hline 19 & 6 & 0 & 5.256186 & -4.044855 & 0.377892 \\
\hline 20 & 16 & 0 & -2.817788 & -2.373951 & 0.156834 \\
\hline 21 & 6 & 0 & -4.426676 & -3.085599 & -0.000123 \\
\hline 22 & 6 & 0 & -4.321585 & -4.486926 & 0.023816 \\
\hline 23 & 6 & 0 & -3.024812 & -4.967708 & 0.149912 \\
\hline 24 & 6 & 0 & -5.649500 & -2.370512 & -0.112623 \\
\hline 25 & 7 & 0 & -4.825939 & -0.032805 & -0.062167 \\
\hline 26 & 6 & 0 & -5.463973 & 1.159217 & -0.025348 \\
\hline
\end{tabular}




\begin{tabular}{|c|c|c|c|c|c|}
\hline 27 & 6 & 0 & -6.916374 & 0.987625 & -0.069183 \\
\hline 28 & 6 & 0 & -7.137342 & -0.346250 & -0.145995 \\
\hline 29 & 6 & 0 & 0.394147 & -2.083778 & -0.355691 \\
\hline 30 & 6 & 0 & -4.848407 & 2.442672 & 0.052409 \\
\hline 31 & 6 & 0 & 0.386875 & 2.434526 & -0.563941 \\
\hline 32 & 6 & 0 & 0.923343 & 1.196558 & -0.995415 \\
\hline 33 & 6 & 0 & 2.294756 & 1.132249 & -0.996432 \\
\hline 34 & 6 & 0 & 2.935376 & 2.332391 & -0.579998 \\
\hline 35 & 16 & 0 & 1.704199 & 3.568997 & -0.233649 \\
\hline 36 & 6 & 0 & -0.958317 & 2.743168 & -0.361477 \\
\hline 37 & 6 & 0 & -1.519978 & 4.010891 & -0.074407 \\
\hline 38 & 6 & 0 & -2.883020 & 3.990553 & 0.085449 \\
\hline 39 & 6 & 0 & -3.481429 & 2.700995 & -0.045685 \\
\hline 40 & 16 & 0 & -2.226651 & 1.498290 & -0.375570 \\
\hline 41 & 6 & 0 & 7.691880 & -2.407047 & 0.055750 \\
\hline 42 & 6 & 0 & 8.358758 & -2.512706 & 1.294695 \\
\hline 43 & 6 & 0 & 9.634130 & -3.085910 & 1.330429 \\
\hline 44 & 6 & 0 & 10.265570 & -3.559378 & 0.177047 \\
\hline 45 & 6 & 0 & 9.587669 & -3.439929 & -1.038928 \\
\hline 46 & 6 & 0 & 8.311197 & -2.873999 & -1.122575 \\
\hline 47 & 6 & 0 & 7.622890 & -2.763229 & -2.464137 \\
\hline 48 & 6 & 0 & 7.723631 & -2.008917 & 2.570735 \\
\hline 49 & 6 & 0 & 11.629412 & -4.204783 & 0.247444 \\
\hline 50 & 6 & 0 & -6.891532 & -3.221574 & -0.206362 \\
\hline 51 & 6 & 0 & -7.380450 & -3.611435 & -1.470920 \\
\hline 52 & 6 & 0 & -8.535310 & -4.397892 & -1.538653 \\
\hline 53 & 6 & 0 & -9.217840 & -4.810663 & -0.391163 \\
\hline 54 & 6 & 0 & -8.717312 & -4.408914 & 0.850109 \\
\hline 55 & 6 & 0 & -7.565304 & -3.623532 & 0.966032 \\
\hline 56 & 6 & 0 & -7.072408 & -3.209560 & 2.333964 \\
\hline 57 & 6 & 0 & -6.685619 & -3.184282 & -2.743882 \\
\hline 58 & 6 & 0 & -10.444726 & -5.686439 & -0.488473 \\
\hline 59 & 6 & 0 & -5.754184 & 3.629035 & 0.261266 \\
\hline 60 & 6 & 0 & -6.213381 & 4.373830 & -0.845442 \\
\hline 61 & 6 & 0 & -7.058398 & 5.467226 & -0.625167 \\
\hline 62 & 6 & 0 & -7.459668 & 5.845265 & 0.658628 \\
\hline 63 & 6 & 0 & -6.988581 & 5.096555 & 1.740797 \\
\hline 64 & 6 & 0 & -6.146813 & 3.993228 & 1.567098 \\
\hline 65 & 6 & 0 & -5.670533 & 3.213697 & 2.771654 \\
\hline 66 & 6 & 0 & -5.810772 & 4.008839 & -2.256505 \\
\hline 67 & 6 & 0 & -8.397910 & 7.009974 & 0.869974 \\
\hline 68 & 6 & 0 & 4.748889 & 4.005426 & -0.200024 \\
\hline 69 & 6 & 0 & 5.019664 & 4.470102 & 1.105201 \\
\hline 70 & 6 & 0 & 5.428315 & 5.796335 & 1.281924 \\
\hline
\end{tabular}




\begin{tabular}{|c|c|c|c|c|c|}
\hline 71 & 6 & 0 & 5.588213 & 6.671052 & 0.204864 \\
\hline 72 & 6 & 0 & 5.319880 & 6.188803 & -1.079533 \\
\hline 73 & 6 & 0 & 4.901203 & 4.874264 & -1.303757 \\
\hline 74 & 6 & 0 & 4.628145 & 4.404350 & -2.714640 \\
\hline 75 & 6 & 0 & 4.873919 & 3.563832 & 2.306585 \\
\hline 76 & 6 & 0 & 6.063237 & 8.089203 & 0.416312 \\
\hline 77 & 1 & 0 & 8.359902 & 0.308139 & -0.274914 \\
\hline 78 & 1 & 0 & 7.212008 & 2.738628 & -0.459524 \\
\hline 79 & 1 & 0 & -0.637699 & -6.372733 & 1.093346 \\
\hline 80 & 1 & 0 & 3.851828 & -5.710780 & 0.682335 \\
\hline 81 & 1 & 0 & 6.198138 & -4.574638 & 0.447512 \\
\hline 82 & 1 & 0 & -5.192875 & -5.122725 & -0.061687 \\
\hline 83 & 1 & 0 & -2.777613 & -6.022179 & 0.158018 \\
\hline 84 & 1 & 0 & -7.645252 & 1.785116 & -0.053816 \\
\hline 85 & 1 & 0 & -8.084800 & -0.862748 & -0.199634 \\
\hline 86 & 1 & 0 & 1.221966 & -1.942803 & -1.050288 \\
\hline 87 & 1 & 0 & -0.537271 & -1.944027 & -0.902800 \\
\hline 88 & 1 & 0 & 0.460498 & -1.336640 & 0.438669 \\
\hline 89 & 1 & 0 & 0.277223 & 0.392492 & -1.325313 \\
\hline 90 & 1 & 0 & 2.881435 & 0.273284 & -1.286448 \\
\hline 91 & 1 & 0 & -0.916767 & 4.909912 & -0.012344 \\
\hline 92 & 1 & 0 & -3.483830 & 4.867013 & 0.291208 \\
\hline 93 & 1 & 0 & 10.146980 & -3.161689 & 2.286792 \\
\hline 94 & 1 & 0 & 10.063632 & -3.795053 & -1.950332 \\
\hline 95 & 1 & 0 & 8.236657 & -3.203290 & -3.254299 \\
\hline 96 & 1 & 0 & 6.654646 & -3.274855 & -2.461909 \\
\hline 97 & 1 & 0 & 7.427377 & -1.718597 & -2.728572 \\
\hline 98 & 1 & 0 & 6.758845 & -2.491738 & 2.758344 \\
\hline 99 & 1 & 0 & 8.371133 & -2.201589 & 3.430004 \\
\hline 100 & 1 & 0 & 7.533555 & -0.931359 & 2.524149 \\
\hline 101 & 1 & 0 & 12.234730 & -3.775075 & 1.051291 \\
\hline 102 & 1 & 0 & 11.548959 & -5.281612 & 0.442316 \\
\hline 103 & 1 & 0 & 12.177979 & -4.085233 & -0.691524 \\
\hline 104 & 1 & 0 & -8.911363 & -4.692499 & -2.515999 \\
\hline 105 & 1 & 0 & -9.237332 & -4.711808 & 1.756290 \\
\hline 106 & 1 & 0 & -7.706900 & -3.628367 & 3.119311 \\
\hline 107 & 1 & 0 & -6.046102 & -3.547665 & 2.511386 \\
\hline 108 & 1 & 0 & -7.069718 & -2.120085 & 2.444659 \\
\hline 109 & 1 & 0 & -5.636802 & -3.498707 & -2.754408 \\
\hline 110 & 1 & 0 & -7.177621 & -3.615459 & -3.619564 \\
\hline 111 & 1 & 0 & -6.689279 & -2.094766 & -2.854902 \\
\hline 112 & 1 & 0 & -11.005416 & -5.490257 & -1.407378 \\
\hline 113 & 1 & 0 & -10.173901 & -6.749782 & -0.494719 \\
\hline 114 & 1 & 0 & -11.117426 & -5.528415 & 0.359702 \\
\hline
\end{tabular}




\begin{tabular}{rrrrrr}
115 & 1 & 0 & -7.411376 & 6.037351 & -1.481795 \\
116 & 1 & 0 & -7.283466 & 5.376581 & 2.749827 \\
117 & 1 & 0 & -6.030301 & 3.669484 & 3.697823 \\
118 & 1 & 0 & -6.026665 & 2.178290 & 2.743275 \\
119 & 1 & 0 & -4.577479 & 3.168730 & 2.816786 \\
120 & 1 & 0 & -6.046120 & 2.963888 & -2.482884 \\
121 & 1 & 0 & -6.328406 & 4.639423 & -2.984123 \\
122 & 1 & 0 & -4.733207 & 4.130049 & -2.412355 \\
123 & 1 & 0 & -8.320329 & 7.739587 & 0.058491 \\
124 & 1 & 0 & -9.442358 & 6.675752 & 0.908333 \\
125 & 1 & 0 & -8.189779 & 7.526634 & 1.811938 \\
126 & 1 & 0 & 5.625842 & 6.153207 & 2.290299 \\
127 & 1 & 0 & 5.438925 & 6.853257 & -1.932698 \\
128 & 1 & 0 & 4.915718 & 5.169285 & -3.440699 \\
129 & 1 & 0 & 5.180968 & 3.488631 & -2.947837 \\
130 & 1 & 0 & 3.566110 & 4.181871 & -2.865904 \\
131 & 1 & 0 & 5.626916 & 2.768048 & 2.299369 \\
132 & 1 & 0 & 4.991120 & 4.128910 & 3.235026 \\
133 & 1 & 0 & 3.895391 & 3.074099 & 2.327392 \\
134 & 1 & 0 & 5.855070 & 8.433410 & 1.433467 \\
135 & 1 & 0 & 7.145834 & 8.172669 & 0.258213 \\
136 & 1 & 0 & 5.581509 & 8.781361 & -0.281368 \\
\hline-----------------------------------------------------------------------------
\end{tabular}

7c (Sum of imaginary frequencies $=0$, total energy $=-4439.19776237$ Hartrees $)$

\begin{tabular}{cccccc}
$\begin{array}{c}\text { Center } \\
\text { Number }\end{array}$ & $\begin{array}{c}\text { Atomic } \\
\text { Number }\end{array}$ & $\begin{array}{l}\text { Atomic } \\
\text { Type }\end{array}$ & \multicolumn{3}{c}{ Coordinates (Angstroms) } \\
\hline 1 & 6 & 0 & -0.187294 & 3.516166 & -1.311155 \\
2 & 6 & 0 & 3.183482 & 2.440443 & -2.126718 \\
3 & 6 & 0 & -3.807565 & 2.844528 & 0.162924 \\
4 & 6 & 0 & -5.061029 & 2.269962 & 0.572396 \\
5 & 6 & 0 & -5.405225 & 0.935736 & 0.423534 \\
6 & 6 & 0 & 6.176090 & -0.579787 & -0.187784 \\
7 & 6 & 0 & -6.739757 & 0.369249 & 0.594180 \\
8 & 6 & 0 & -6.675262 & -0.899276 & 0.109339 \\
9 & 6 & 0 & -5.285636 & -1.109842 & -0.298315 \\
10 & 7 & 0 & -4.545254 & -0.019564 & -0.090444 \\
11 & 6 & 0 & -4.773636 & -2.308198 & -0.925745 \\
12 & 7 & 0 & 0.706771 & 2.492832 & -1.552888 \\
13 & 6 & 0 & 1.897547 & 3.088022 & -1.964768 \\
14 & 6 & 0 & 1.628103 & 4.452662 & -1.968523 \\
15 & 7 & 0 & 0.363163 & 4.707654 & -1.566731 \\
16 & 16 & 0 & -2.460042 & 1.941172 & -0.528592
\end{tabular}




\begin{tabular}{|c|c|c|c|c|c|}
\hline 17 & 6 & 0 & -1.530190 & 3.410231 & -0.768798 \\
\hline 18 & 6 & 0 & -2.240479 & 4.523491 & -0.339892 \\
\hline 19 & 6 & 0 & -3.499840 & 4.206010 & 0.185442 \\
\hline 20 & 16 & 0 & 3.670077 & 1.099033 & -1.110718 \\
\hline 21 & 6 & 0 & 5.329833 & 1.168464 & -1.711985 \\
\hline 22 & 6 & 0 & 5.436242 & 2.174181 & -2.672102 \\
\hline 23 & 6 & 0 & 4.241236 & 2.881024 & -2.911042 \\
\hline 24 & 6 & 0 & 6.408468 & 0.394768 & -1.146832 \\
\hline 25 & 7 & 0 & 4.879852 & -0.928922 & 0.125714 \\
\hline 26 & 6 & 0 & 4.925762 & -1.658687 & 1.234551 \\
\hline 27 & 6 & 0 & 6.306460 & -1.831655 & 1.684596 \\
\hline 28 & 6 & 0 & 7.092175 & -1.192718 & 0.767116 \\
\hline 29 & 6 & 0 & 0.414474 & 1.060905 & -1.518815 \\
\hline 30 & 6 & 0 & 3.678128 & -2.081340 & 1.860771 \\
\hline 31 & 6 & 0 & -0.938961 & -2.977244 & -0.493831 \\
\hline 32 & 6 & 0 & -3.437011 & -2.655632 & -0.910297 \\
\hline 33 & 6 & 0 & 0.379493 & -2.950400 & -0.070071 \\
\hline 34 & 6 & 0 & 1.520188 & -3.297090 & -0.861654 \\
\hline 35 & 6 & 0 & 2.716653 & -2.954081 & -0.305286 \\
\hline 36 & 6 & 0 & 2.629269 & -2.426581 & 1.029852 \\
\hline 37 & 16 & 0 & 0.912809 & -2.324095 & 1.500938 \\
\hline 38 & 6 & 0 & -1.464889 & -3.781386 & -1.552628 \\
\hline 39 & 6 & 0 & -2.810251 & -3.644821 & -1.743235 \\
\hline 40 & 16 & 0 & -2.213650 & -1.941395 & 0.169947 \\
\hline 41 & 6 & 0 & -6.080979 & 3.216646 & 1.143512 \\
\hline 42 & 6 & 0 & -6.111743 & 3.447783 & 2.534724 \\
\hline 43 & 6 & 0 & -7.065547 & 4.329745 & 3.053150 \\
\hline 44 & 6 & 0 & -7.987870 & 4.987094 & 2.234884 \\
\hline 45 & 6 & 0 & -7.934550 & 4.745643 & 0.859493 \\
\hline 46 & 6 & 0 & -6.998853 & 3.871839 & 0.295845 \\
\hline 47 & 6 & 0 & -6.991782 & 3.639742 & -1.198210 \\
\hline 48 & 6 & 0 & -5.136999 & 2.760482 & 3.463758 \\
\hline 49 & 6 & 0 & -9.028111 & 5.911822 & 2.821724 \\
\hline 50 & 6 & 0 & 7.810475 & 0.739368 & -1.551611 \\
\hline 51 & 6 & 0 & 8.445617 & -0.011283 & -2.562994 \\
\hline 52 & 6 & 0 & 9.754428 & 0.318659 & -2.929535 \\
\hline 53 & 6 & 0 & 10.451592 & 1.365396 & -2.320886 \\
\hline 54 & 6 & 0 & 9.804159 & 2.089100 & -1.315660 \\
\hline 55 & 6 & 0 & 8.494187 & 1.800866 & -0.920516 \\
\hline 56 & 6 & 0 & 7.843713 & 2.610391 & 0.178007 \\
\hline 57 & 6 & 0 & 7.739534 & -1.159869 & -3.248135 \\
\hline 58 & 6 & 0 & 11.854016 & 1.721462 & -2.754025 \\
\hline 59 & 6 & 0 & 3.535219 & -2.004947 & 3.343947 \\
\hline 60 & 6 & 0 & 3.313280 & -3.184276 & 4.096815 \\
\hline
\end{tabular}




\begin{tabular}{|c|c|c|c|c|c|}
\hline 61 & 6 & 0 & 3.200879 & -3.093126 & 5.487097 \\
\hline 62 & 6 & 0 & 3.289519 & -1.872427 & 6.160418 \\
\hline 63 & 6 & 0 & 3.513262 & -0.720544 & 5.402086 \\
\hline 64 & 6 & 0 & 3.644170 & -0.760785 & 4.011465 \\
\hline 65 & 6 & 0 & 3.880624 & 0.528457 & 3.257306 \\
\hline 66 & 6 & 0 & 3.218530 & -4.544761 & 3.442807 \\
\hline 67 & 6 & 0 & 3.121475 & -1.796446 & 7.659003 \\
\hline 68 & 6 & 0 & -5.745467 & -3.169723 & -1.678009 \\
\hline 69 & 6 & 0 & -6.138940 & -4.422402 & -1.155864 \\
\hline 70 & 6 & 0 & -7.056257 & -5.200094 & -1.870610 \\
\hline 71 & 6 & 0 & -7.593931 & -4.778475 & -3.089249 \\
\hline 72 & 6 & 0 & -7.188824 & -3.538676 & -3.591064 \\
\hline 73 & 6 & 0 & -6.282160 & -2.723213 & -2.907179 \\
\hline 74 & 6 & 0 & -5.883668 & -1.390486 & -3.499373 \\
\hline 75 & 6 & 0 & -5.599601 & -4.935946 & 0.161002 \\
\hline 76 & 6 & 0 & -8.604199 & -5.624028 & -3.828016 \\
\hline 77 & 1 & 0 & -7.600205 & 0.889431 & 0.991737 \\
\hline 78 & 1 & 0 & -7.469408 & -1.630297 & 0.040599 \\
\hline 79 & 1 & 0 & 2.325792 & 5.246247 & -2.198969 \\
\hline 80 & 1 & 0 & -1.828366 & 5.521488 & -0.409568 \\
\hline 81 & 1 & 0 & -4.194134 & 4.942126 & 0.570974 \\
\hline 82 & 1 & 0 & 6.373142 & 2.390980 & -3.171658 \\
\hline 83 & 1 & 0 & 4.154186 & 3.695918 & -3.620418 \\
\hline 84 & 1 & 0 & 6.623155 & -2.380083 & 2.562105 \\
\hline 85 & 1 & 0 & 8.170556 & -1.105295 & 0.762313 \\
\hline 86 & 1 & 0 & -0.508091 & 0.856436 & -2.065266 \\
\hline 87 & 1 & 0 & 1.226887 & 0.519762 & -1.999481 \\
\hline 88 & 1 & 0 & 0.308912 & 0.700049 & -0.492435 \\
\hline 89 & 1 & 0 & 1.416215 & -3.715396 & -1.856200 \\
\hline 90 & 1 & 0 & 3.675233 & -3.086262 & -0.787083 \\
\hline 91 & 1 & 0 & -0.846306 & -4.482884 & -2.100655 \\
\hline 92 & 1 & 0 & -3.383515 & -4.201131 & -2.474505 \\
\hline 93 & 1 & 0 & -7.085043 & 4.507266 & 4.126174 \\
\hline 94 & 1 & 0 & -8.639110 & 5.251690 & 0.203067 \\
\hline 95 & 1 & 0 & -7.715420 & 4.290390 & -1.695963 \\
\hline 96 & 1 & 0 & -6.005533 & 3.834298 & -1.631444 \\
\hline 97 & 1 & 0 & -7.246602 & 2.602590 & -1.441888 \\
\hline 98 & 1 & 0 & -5.237253 & 1.671249 & 3.414295 \\
\hline 99 & 1 & 0 & -4.099410 & 2.993646 & 3.201931 \\
\hline 100 & 1 & 0 & -5.302010 & 3.069800 & 4.499008 \\
\hline 101 & 1 & 0 & -8.673664 & 6.378082 & 3.745794 \\
\hline 102 & 1 & 0 & -9.296814 & 6.708040 & 2.121016 \\
\hline 103 & 1 & 0 & -9.949945 & 5.368461 & 3.064636 \\
\hline 104 & 1 & 0 & 10.240531 & -0.261604 & -3.710758 \\
\hline
\end{tabular}




\begin{tabular}{rrrrrr}
105 & 1 & 0 & 10.332466 & 2.901370 & -0.821476 \\
106 & 1 & 0 & 8.537930 & 3.354619 & 0.576433 \\
107 & 1 & 0 & 6.954489 & 3.136988 & -0.184057 \\
108 & 1 & 0 & 7.518310 & 1.972196 & 1.006155 \\
109 & 1 & 0 & 6.773013 & -0.851064 & -3.659786 \\
110 & 1 & 0 & 8.346155 & -1.555138 & -4.066897 \\
111 & 1 & 0 & 7.538373 & -1.979517 & -2.550113 \\
112 & 1 & 0 & 12.386215 & 0.849062 & -3.144474 \\
113 & 1 & 0 & 11.843243 & 2.478364 & -3.548357 \\
114 & 1 & 0 & 12.437165 & 2.131834 & -1.924194 \\
115 & 1 & 0 & 3.043284 & -4.004691 & 6.059426 \\
116 & 1 & 0 & 3.584472 & 0.241040 & 5.905869 \\
117 & 1 & 0 & 3.643630 & 1.390297 & 3.887038 \\
118 & 1 & 0 & 4.926684 & 0.623732 & 2.944220 \\
119 & 1 & 0 & 3.274219 & 0.588181 & 2.349781 \\
120 & 1 & 0 & 3.999810 & -4.690576 & 2.691283 \\
121 & 1 & 0 & 3.311569 & -5.336915 & 4.190677 \\
122 & 1 & 0 & 2.257494 & -4.681260 & 2.934280 \\
123 & 1 & 0 & 3.444148 & -2.721672 & 8.145796 \\
124 & 1 & 0 & 3.696350 & -0.968941 & 8.085557 \\
125 & 1 & 0 & 2.070550 & -1.635836 & 7.930707 \\
126 & 1 & 0 & -7.358884 & -6.161221 & -1.460300 \\
127 & 1 & 0 & -7.587275 & -3.195713 & -4.543532 \\
128 & 1 & 0 & -6.224537 & -1.308126 & -4.535018 \\
129 & 1 & 0 & -6.319037 & -0.557094 & -2.936629 \\
130 & 1 & 0 & -4.799220 & -1.247257 & -3.481051 \\
131 & 1 & 0 & -5.646986 & -4.171412 & 0.942358 \\
132 & 1 & 0 & -6.169860 & -5.805586 & 0.498387 \\
133 & 1 & 0 & -4.549286 & -5.237155 & 0.079333 \\
134 & 1 & 0 & -8.458165 & -6.689721 & -3.627789 \\
135 & 1 & 0 & -9.628581 & -5.373968 & -3.524241 \\
136 & 1 & 0 & -8.541044 & -5.469807 & -4.909472 \\
----------------------------------------------------------------------------------- \\
\hline
\end{tabular}

$(M, M)-8$ (The X-ray crystallographic structure of $\mathbf{8}$ were used for optimization, sum of imaginary frequencies $=0$, total energy $=-8878.47213581$ Hartrees)

\begin{tabular}{|c|c|c|c|c|c|}
\hline Center & Atomic & Atomic & \multicolumn{3}{|c|}{ Coordinates (Angstroms) } \\
\hline Number & Number & Type & $X$ & $\mathrm{Y}$ & Z \\
\hline 1 & 6 & 0 & 0.351105 & 27.553494 & 12.304614 \\
\hline 2 & 16 & 0 & 0.974152 & 25.199426 & 9.627364 \\
\hline 3 & 7 & 0 & 3.376529 & 29.352590 & 9.391305 \\
\hline 4 & 6 & 0 & -0.821402 & 27.523973 & 13.066338 \\
\hline
\end{tabular}


48

\begin{tabular}{rrr}
-0.748622 & 27.323517 & 14.133059 \\
2.882253 & 22.064524 & 7.104540 \\
4.193014 & 20.005420 & 5.511167 \\
-2.077972 & 27.746522 & 12.497425 \\
5.526392 & 22.523601 & 4.815050 \\
5.296748 & 25.484789 & 5.436300 \\
-2.141539 & 28.012368 & 11.126793 \\
-3.109046 & 28.198643 & 10.665531 \\
6.642133 & 26.239653 & 3.811297 \\
7.269380 & 29.516390 & 3.047015 \\
-0.995588 & 28.050121 & 10.326261 \\
6.043802 & 28.968370 & 8.224390 \\
8.526743 & 26.770273 & 7.362288 \\
0.263485 & 27.814932 & 10.920406 \\
11.633990 & 24.518961 & 6.198004 \\
9.994455 & 27.021757 & 5.688174 \\
1.687642 & 27.313585 & 12.968604 \\
2.353471 & 28.175552 & 12.852724 \\
2.203362 & 26.452867 & 12.529961 \\
1.562920 & 27.123907 & 14.037975 \\
13.976989 & 25.856644 & 3.917639 \\
14.203834 & 23.346053 & 5.396534 \\
-3.333836 & 27.677581 & 13.333861 \\
-4.104619 & 28.356394 & 12.956256 \\
-3.134569 & 27.937096 & 14.377867 \\
-3.760260 & 26.666562 & 13.325740 \\
13.573781 & 29.621092 & 1.572312 \\
9.151592 & 31.468671 & 1.923088 \\
-1.118014 & 28.352624 & 8.850429 \\
-0.748513 & 27.521698 & 8.240306 \\
-0.534802 & 29.237136 & 8.572528 \\
-2.160437 & 28.534871 & 8.576263 \\
1.515508 & 27.857568 & 10.086501 \\
1.918797 & 26.696849 & 9.449584 \\
3.026412 & 26.456002 & 8.570482 \\
3.718570 & 27.255349 & 8.345788 \\
3.092621 & 25.183088 & 8.071062 \\
3.849969 & 24.858342 & 7.362707 \\
2.047134 & 24.318358 & 8.522085 \\
1.820340 & 22.993749 & 8.188056 \\
0.034139 & 22.178924 & 8.628371 \\
0.010962 & 20.153843 & 8.351325 \\
\hline & 22.552654 & 9.297917 \\
\hline
\end{tabular}




\begin{tabular}{|c|c|c|c|c|c|}
\hline 49 & 6 & 0 & 1.867038 & 20.621740 & 7.266958 \\
\hline 50 & 6 & 0 & 2.114036 & 19.393985 & 6.671556 \\
\hline 51 & 6 & 0 & 1.126765 & 18.281397 & 6.903134 \\
\hline 52 & 6 & 0 & 1.283360 & 17.403884 & 7.996863 \\
\hline 53 & 6 & 0 & 0.352028 & 16.376959 & 8.184144 \\
\hline 54 & 1 & 0 & 0.476140 & 15.705575 & 9.031228 \\
\hline 55 & 6 & 0 & -0.728529 & 16.191671 & 7.317365 \\
\hline 56 & 6 & 0 & -0.865827 & 17.072917 & 6.241742 \\
\hline 57 & 1 & 0 & -1.702535 & 16.949574 & 5.557553 \\
\hline 58 & 6 & 0 & 0.042009 & 18.113200 & 6.016343 \\
\hline 59 & 6 & 0 & 2.435331 & 17.559078 & 8.963920 \\
\hline 60 & 1 & 0 & 2.410750 & 16.776100 & 9.726402 \\
\hline 61 & 1 & 0 & 2.407277 & 18.528367 & 9.473134 \\
\hline 62 & 1 & 0 & 3.400532 & 17.502112 & 8.449535 \\
\hline 63 & 6 & 0 & -1.702777 & 15.055523 & 7.522147 \\
\hline 64 & 1 & 0 & -1.830884 & 14.823780 & 8.583918 \\
\hline 65 & 1 & 0 & -2.686377 & 15.291681 & 7.105445 \\
\hline 66 & 1 & 0 & -1.352913 & 14.138982 & 7.030576 \\
\hline 67 & 6 & 0 & -0.152601 & 19.040183 & 4.838120 \\
\hline 68 & 1 & 0 & -1.016627 & 18.736676 & 4.241020 \\
\hline 69 & 1 & 0 & 0.725868 & 19.047994 & 4.184459 \\
\hline 70 & 1 & 0 & -0.311617 & 20.074564 & 5.161375 \\
\hline 71 & 6 & 0 & 3.258901 & 19.108909 & 5.855339 \\
\hline 72 & 6 & 0 & 3.572737 & 17.779173 & 5.323240 \\
\hline 73 & 1 & 0 & 2.971805 & 16.890359 & 5.454700 \\
\hline 74 & 6 & 0 & 4.748789 & 17.904935 & 4.660724 \\
\hline 75 & 1 & 0 & 5.302391 & 17.141814 & 4.132086 \\
\hline 76 & 6 & 0 & 5.135691 & 19.309720 & 4.776018 \\
\hline 77 & 6 & 0 & 6.279468 & 19.866353 & 4.219126 \\
\hline 78 & 6 & 0 & 7.247577 & 18.948813 & 3.525102 \\
\hline 79 & 6 & 0 & 7.226060 & 18.825907 & 2.118719 \\
\hline 80 & 6 & 0 & 8.119493 & 17.944641 & 1.501930 \\
\hline 81 & 1 & 0 & 8.093273 & 17.846202 & 0.418907 \\
\hline 82 & 6 & 0 & 9.034808 & 17.184042 & 2.234885 \\
\hline 83 & 6 & 0 & 9.034300 & 17.318877 & 3.625584 \\
\hline 84 & 1 & 0 & 9.728903 & 16.726104 & 4.217009 \\
\hline 85 & 6 & 0 & 8.159616 & 18.188592 & 4.286923 \\
\hline 86 & 6 & 0 & 8.194278 & 18.285309 & 5.795663 \\
\hline 87 & 1 & 0 & 9.094402 & 17.814473 & 6.197891 \\
\hline 88 & 1 & 0 & 7.324675 & 17.794670 & 6.246817 \\
\hline 89 & 1 & 0 & 8.179590 & 19.326167 & 6.132290 \\
\hline 90 & 6 & 0 & 10.010855 & 16.263104 & 1.541799 \\
\hline 91 & 1 & 0 & 9.588853 & 15.853619 & 0.619036 \\
\hline 92 & 1 & 0 & 10.931631 & 16.794018 & 1.269383 \\
\hline
\end{tabular}




\begin{tabular}{|c|c|c|c|c|c|}
\hline 93 & 1 & 0 & 10.297490 & 15.425449 & 2.184670 \\
\hline 94 & 6 & 0 & 6.243907 & 19.608100 & 1.276629 \\
\hline 95 & 1 & 0 & 6.294974 & 19.296733 & 0.230122 \\
\hline 96 & 1 & 0 & 6.445624 & 20.683852 & 1.318768 \\
\hline 97 & 1 & 0 & 5.216093 & 19.465531 & 1.625295 \\
\hline 98 & 6 & 0 & 6.607449 & 21.264081 & 4.239423 \\
\hline 99 & 6 & 0 & 7.787763 & 21.830400 & 3.751253 \\
\hline 100 & 1 & 0 & 8.599283 & 21.227623 & 3.363014 \\
\hline 101 & 6 & 0 & 7.819870 & 23.233767 & 3.814203 \\
\hline 102 & 1 & 0 & 8.679519 & 23.819356 & 3.515060 \\
\hline 103 & 6 & 0 & 6.657459 & 23.775681 & 4.348476 \\
\hline 104 & 6 & 0 & 6.241296 & 25.145251 & 4.550718 \\
\hline 105 & 6 & 0 & 5.892829 & 27.324500 & 4.255516 \\
\hline 106 & 6 & 0 & 5.079275 & 26.807205 & 5.260530 \\
\hline 107 & 1 & 0 & 4.374660 & 27.356625 & 5.867498 \\
\hline 108 & 6 & 0 & 7.581695 & 26.235474 & 2.695886 \\
\hline 109 & 1 & 0 & 7.307241 & 27.019949 & 1.991204 \\
\hline 110 & 1 & 0 & 7.527324 & 25.272246 & 2.188240 \\
\hline 111 & 1 & 0 & 8.604101 & 26.407955 & 3.045676 \\
\hline 112 & 6 & 0 & 5.899210 & 28.681232 & 3.764323 \\
\hline 113 & 6 & 0 & 4.814785 & 29.542248 & 3.886189 \\
\hline 114 & 1 & 0 & 3.859862 & 29.226651 & 4.288346 \\
\hline 115 & 6 & 0 & 5.070583 & 30.835960 & 3.414657 \\
\hline 116 & 1 & 0 & 4.338639 & 31.633535 & 3.417547 \\
\hline 117 & 6 & 0 & 6.357360 & 31.019218 & 2.905321 \\
\hline 118 & 6 & 0 & 6.855728 & 32.229595 & 2.322260 \\
\hline 119 & 6 & 0 & 5.886360 & 33.377839 & 2.243060 \\
\hline 120 & 6 & 0 & 5.833075 & 34.321629 & 3.290481 \\
\hline 121 & 6 & 0 & 4.915558 & 35.374515 & 3.206830 \\
\hline 122 & 1 & 0 & 4.875911 & 36.099686 & 4.016865 \\
\hline 123 & 6 & 0 & 4.055578 & 35.522024 & 2.115131 \\
\hline 124 & 6 & 0 & 4.125937 & 34.574006 & 1.090970 \\
\hline 125 & 1 & 0 & 3.462535 & 34.667461 & 0.233952 \\
\hline 126 & 6 & 0 & 5.024369 & 33.502635 & 1.133418 \\
\hline 127 & 6 & 0 & 5.062049 & 32.506456 & -0.003124 \\
\hline 128 & 1 & 0 & 4.329058 & 32.764791 & -0.771625 \\
\hline 129 & 1 & 0 & 4.844271 & 31.491212 & 0.345066 \\
\hline 130 & 1 & 0 & 6.050441 & 32.474237 & -0.473324 \\
\hline 131 & 6 & 0 & 6.751997 & 34.218715 & 4.487054 \\
\hline 132 & 1 & 0 & 6.469787 & 34.939987 & 5.258368 \\
\hline 133 & 1 & 0 & 7.792800 & 34.413295 & 4.205245 \\
\hline 134 & 1 & 0 & 6.726327 & 33.219383 & 4.932742 \\
\hline 135 & 6 & 0 & 3.097860 & 36.687069 & 2.031599 \\
\hline 136 & 1 & 0 & 2.212540 & 36.436289 & 1.439740 \\
\hline
\end{tabular}




\begin{tabular}{|c|c|c|c|c|c|}
\hline 137 & 1 & 0 & 3.570999 & 37.555662 & 1.556568 \\
\hline 138 & 1 & 0 & 2.763881 & 37.003711 & 3.024338 \\
\hline 139 & 6 & 0 & 8.145277 & 32.413747 & 1.844151 \\
\hline 140 & 6 & 0 & 8.626321 & 33.614483 & 1.169797 \\
\hline 141 & 1 & 0 & 8.044921 & 34.504675 & 0.975003 \\
\hline 142 & 6 & 0 & 9.916547 & 33.365278 & 0.833957 \\
\hline 143 & 1 & 0 & 10.607340 & 34.014352 & 0.314939 \\
\hline 144 & 6 & 0 & 10.221360 & 32.019258 & 1.332824 \\
\hline 145 & 6 & 0 & 11.531608 & 31.425687 & 1.204195 \\
\hline 146 & 6 & 0 & 12.549827 & 32.179250 & 0.391672 \\
\hline 147 & 6 & 0 & 13.401548 & 33.119296 & 1.014612 \\
\hline 148 & 6 & 0 & 14.337304 & 33.806622 & 0.237548 \\
\hline 149 & 1 & 0 & 14.990633 & 34.529405 & 0.721953 \\
\hline 150 & 6 & 0 & 14.456222 & 33.592152 & -1.139649 \\
\hline 151 & 6 & 0 & 13.603307 & 32.661474 & -1.735163 \\
\hline 152 & 1 & 0 & 13.678206 & 32.480397 & -2.805066 \\
\hline 153 & 6 & 0 & 12.649738 & 31.951154 & -0.996240 \\
\hline 154 & 6 & 0 & 13.306667 & 33.395300 & 2.497850 \\
\hline 155 & 1 & 0 & 14.040527 & 34.146719 & 2.800928 \\
\hline 156 & 1 & 0 & 13.487025 & 32.490121 & 3.086961 \\
\hline 157 & 1 & 0 & 12.312394 & 33.763047 & 2.774201 \\
\hline 158 & 6 & 0 & 15.474013 & 34.356103 & -1.953606 \\
\hline 159 & 1 & 0 & 15.503132 & 34.005022 & -2.988692 \\
\hline 160 & 1 & 0 & 16.480883 & 34.250330 & -1.534567 \\
\hline 161 & 1 & 0 & 15.246243 & 35.428555 & -1.972307 \\
\hline 162 & 6 & 0 & 11.746266 & 30.958156 & -1.691001 \\
\hline 163 & 1 & 0 & 11.963472 & 30.917505 & -2.761626 \\
\hline 164 & 1 & 0 & 10.690393 & 31.222745 & -1.569139 \\
\hline 165 & 1 & 0 & 11.870253 & 29.949733 & -1.282361 \\
\hline 166 & 6 & 0 & 11.917465 & 30.232972 & 1.790879 \\
\hline 167 & 6 & 0 & 11.190265 & 29.336369 & 2.642172 \\
\hline 168 & 1 & 0 & 10.157428 & 29.544691 & 2.883867 \\
\hline 169 & 6 & 0 & 11.912457 & 28.263118 & 3.090748 \\
\hline 170 & 1 & 0 & 11.513634 & 27.515815 & 3.771521 \\
\hline 171 & 6 & 0 & 13.261816 & 28.222995 & 2.619542 \\
\hline 172 & 6 & 0 & 14.243251 & 27.283955 & 2.889348 \\
\hline 173 & 6 & 0 & 15.586538 & 27.297011 & 2.406613 \\
\hline 174 & 1 & 0 & 15.959621 & 28.084972 & 1.761824 \\
\hline 175 & 6 & 0 & 16.338978 & 26.239295 & 2.827791 \\
\hline 176 & 1 & 0 & 17.376738 & 26.083832 & 2.561392 \\
\hline 177 & 6 & 0 & 15.642283 & 25.305462 & 3.668265 \\
\hline 178 & 6 & 0 & 16.190258 & 24.140454 & 4.183571 \\
\hline 179 & 6 & 0 & 17.625577 & 23.826715 & 3.855473 \\
\hline 180 & 6 & 0 & 18.654572 & 24.233670 & 4.733213 \\
\hline
\end{tabular}




\begin{tabular}{|c|c|c|c|c|c|}
\hline 181 & 6 & 0 & 19.980086 & 23.923356 & 4.417465 \\
\hline 182 & 1 & 0 & 20.768224 & 24.239035 & 5.098070 \\
\hline 183 & 6 & 0 & 20.319222 & 23.220267 & 3.256632 \\
\hline 184 & 6 & 0 & 19.286512 & 22.823969 & 2.404923 \\
\hline 185 & 1 & 0 & 19.527061 & 22.271731 & 1.499213 \\
\hline 186 & 6 & 0 & 17.944716 & 23.113625 & 2.682036 \\
\hline 187 & 6 & 0 & 18.340316 & 24.993280 & 6.002002 \\
\hline 188 & 1 & 0 & 19.255248 & 25.227621 & 6.552451 \\
\hline 189 & 1 & 0 & 17.685429 & 24.415531 & 6.663030 \\
\hline 190 & 1 & 0 & 17.821028 & 25.933896 & 5.790121 \\
\hline 191 & 6 & 0 & 21.762650 & 22.910759 & 2.936112 \\
\hline 192 & 1 & 0 & 21.846959 & 22.251505 & 2.067866 \\
\hline 193 & 1 & 0 & 22.263722 & 22.422553 & 3.779251 \\
\hline 194 & 1 & 0 & 22.326397 & 23.824940 & 2.714675 \\
\hline 195 & 6 & 0 & 16.865118 & 22.658400 & 1.726402 \\
\hline 196 & 1 & 0 & 17.291361 & 22.073505 & 0.907007 \\
\hline 197 & 1 & 0 & 16.328198 & 23.508075 & 1.290944 \\
\hline 198 & 1 & 0 & 16.116418 & 22.039738 & 2.232150 \\
\hline 199 & 6 & 0 & 15.471832 & 23.196660 & 4.990477 \\
\hline 200 & 6 & 0 & 16.031938 & 21.920202 & 5.445319 \\
\hline 201 & 1 & 0 & 17.036158 & 21.569164 & 5.253821 \\
\hline 202 & 6 & 0 & 15.042144 & 21.294929 & 6.128370 \\
\hline 203 & 1 & 0 & 15.073713 & 20.331165 & 6.616637 \\
\hline 204 & 6 & 0 & 13.891651 & 22.195917 & 6.098677 \\
\hline 205 & 6 & 0 & 12.664479 & 21.933824 & 6.692934 \\
\hline 206 & 6 & 0 & 12.467001 & 20.594208 & 7.347138 \\
\hline 207 & 6 & 0 & 12.617300 & 20.456377 & 8.744658 \\
\hline 208 & 6 & 0 & 12.444026 & 19.195773 & 9.323292 \\
\hline 209 & 1 & 0 & 12.562251 & 19.093641 & 10.399912 \\
\hline 210 & 6 & 0 & 12.129959 & 18.066986 & 8.560194 \\
\hline 211 & 6 & 0 & 11.985756 & 18.228073 & 7.180389 \\
\hline 212 & 1 & 0 & 11.744638 & 17.362720 & 6.566730 \\
\hline 213 & 6 & 0 & 12.147982 & 19.471184 & 6.556741 \\
\hline 214 & 6 & 0 & 12.979394 & 21.638427 & 9.614947 \\
\hline 215 & 1 & 0 & 13.897576 & 22.122610 & 9.266768 \\
\hline 216 & 1 & 0 & 12.195470 & 22.402898 & 9.603531 \\
\hline 217 & 1 & 0 & 13.131496 & 21.326406 & 10.651379 \\
\hline 218 & 6 & 0 & 11.979944 & 16.711082 & 9.208679 \\
\hline 219 & 1 & 0 & 11.405089 & 16.026870 & 8.578107 \\
\hline 220 & 1 & 0 & 11.475210 & 16.783918 & 10.177211 \\
\hline 221 & 1 & 0 & 12.957752 & 16.247177 & 9.388883 \\
\hline 222 & 6 & 0 & 11.993063 & 19.585299 & 5.057028 \\
\hline 223 & 1 & 0 & 11.532898 & 18.686037 & 4.641051 \\
\hline 224 & 1 & 0 & 12.962698 & 19.728003 & 4.567211 \\
\hline
\end{tabular}




\begin{tabular}{|c|c|c|c|c|c|}
\hline 225 & 1 & 0 & 11.370370 & 20.441374 & 4.780383 \\
\hline 226 & 6 & 0 & 11.560022 & 22.849687 & 6.741543 \\
\hline 227 & 6 & 0 & 10.299076 & 22.574265 & 7.276743 \\
\hline 228 & 1 & 0 & 10.034173 & 21.594920 & 7.655213 \\
\hline 229 & 6 & 0 & 9.422315 & 23.671970 & 7.274055 \\
\hline 230 & 1 & 0 & 8.397431 & 23.610099 & 7.616105 \\
\hline 231 & 6 & 0 & 9.995633 & 24.818069 & 6.737618 \\
\hline 232 & 6 & 0 & 9.485252 & 26.160957 & 6.577810 \\
\hline 233 & 6 & 0 & 7.825386 & 26.175930 & 8.494335 \\
\hline 234 & 1 & 0 & 7.578185 & 26.956285 & 9.213425 \\
\hline 235 & 1 & 0 & 8.477880 & 25.447332 & 8.976167 \\
\hline 236 & 1 & 0 & 6.903610 & 25.683852 & 8.169516 \\
\hline 237 & 6 & 0 & 8.442146 & 28.094085 & 6.942972 \\
\hline 238 & 6 & 0 & 9.366564 & 28.198953 & 5.907358 \\
\hline 239 & 1 & 0 & 9.568848 & 29.075248 & 5.309392 \\
\hline 240 & 6 & 0 & 7.625701 & 29.155380 & 7.481899 \\
\hline 241 & 6 & 0 & 7.964438 & 30.499928 & 7.390425 \\
\hline 242 & 1 & 0 & 8.907226 & 30.838840 & 6.978647 \\
\hline 243 & 6 & 0 & 6.986638 & 31.360170 & 7.906566 \\
\hline 244 & 1 & 0 & 7.085064 & 32.437822 & 7.932948 \\
\hline 245 & 6 & 0 & 5.861641 & 30.711601 & 8.418086 \\
\hline 246 & 6 & 0 & 4.741591 & 31.357099 & 9.037751 \\
\hline 247 & 6 & 0 & 4.823676 & 32.854369 & 9.162737 \\
\hline 248 & 6 & 0 & 5.443085 & 33.439122 & 10.287494 \\
\hline 249 & 6 & 0 & 5.511034 & 34.833385 & 10.374970 \\
\hline 250 & 1 & 0 & 5.983394 & 35.280456 & 11.246943 \\
\hline 251 & 6 & 0 & 4.989971 & 35.663874 & 9.378850 \\
\hline 252 & 6 & 0 & 4.379212 & 35.061954 & 8.275638 \\
\hline 253 & 1 & 0 & 3.957874 & 35.689097 & 7.492892 \\
\hline 254 & 6 & 0 & 4.285148 & 33.671750 & 8.146880 \\
\hline 255 & 6 & 0 & 6.016229 & 32.586125 & 11.395964 \\
\hline 256 & 1 & 0 & 6.451848 & 33.208911 & 12.181499 \\
\hline 257 & 1 & 0 & 6.797775 & 31.915662 & 11.023079 \\
\hline 258 & 1 & 0 & 5.247278 & 31.953187 & 11.851165 \\
\hline 259 & 6 & 0 & 5.106656 & 37.166456 & 9.480610 \\
\hline 260 & 1 & 0 & 4.297885 & 37.668287 & 8.941180 \\
\hline 261 & 1 & 0 & 6.052693 & 37.521148 & 9.052534 \\
\hline 262 & 1 & 0 & 5.077985 & 37.500473 & 10.522152 \\
\hline 263 & 6 & 0 & 3.600304 & 33.073399 & 6.938824 \\
\hline 264 & 1 & 0 & 3.363630 & 33.845736 & 6.202305 \\
\hline 265 & 1 & 0 & 2.666202 & 32.573746 & 7.218262 \\
\hline 266 & 1 & 0 & 4.228019 & 32.321429 & 6.450199 \\
\hline 267 & 6 & 0 & 3.609297 & 30.710440 & 9.511085 \\
\hline 268 & 6 & 0 & 2.510152 & 31.354543 & 10.222075 \\
\hline
\end{tabular}




$\begin{array}{rrrrrr}269 & 1 & 0 & 2.438710 & 32.408475 & 10.451676 \\ 270 & 6 & 0 & 1.637037 & 30.366008 & 10.538276 \\ 271 & 1 & 0 & 0.701558 & 30.449275 & 11.073009 \\ 272 & 6 & 0 & 2.200061 & 29.125789 & 9.991025\end{array}$

$(P, P)-\mathbf{8}$ (The X-ray crystallographic structure of $\mathbf{8}$ were used for optimization, sum of imaginary frequencies $=0$, total energy $=-8878.47216035$ Hartrees)

\begin{tabular}{|c|c|c|c|c|c|}
\hline \multirow{2}{*}{$\begin{array}{l}\text { Center } \\
\text { Number }\end{array}$} & \multirow{2}{*}{$\begin{array}{l}\text { Atomic } \\
\text { Number }\end{array}$} & \multirow{2}{*}{$\begin{array}{l}\text { Atomic } \\
\text { Type }\end{array}$} & \multicolumn{3}{|c|}{ Coordinates (Angstroms) } \\
\hline & & & $\mathrm{X}$ & Y & $\mathrm{Z}$ \\
\hline 1 & 6 & 0 & 0.372649 & 30.532625 & 22.355849 \\
\hline 2 & 16 & 0 & 0.990223 & 32.887452 & 19.678228 \\
\hline 3 & 7 & 0 & 3.395194 & 28.735919 & 19.437665 \\
\hline 4 & 6 & 0 & -0.798860 & 30.561299 & 23.119144 \\
\hline 5 & 1 & 0 & -0.724776 & 30.761696 & 24.185786 \\
\hline 6 & 16 & 0 & 2.893083 & 36.024395 & 17.154020 \\
\hline 7 & 7 & 0 & 4.200398 & 38.084366 & 15.559306 \\
\hline 8 & 6 & 0 & -2.056042 & 30.337975 & 22.551894 \\
\hline 9 & 16 & 0 & 5.532025 & 35.566676 & 14.858711 \\
\hline 10 & 7 & 0 & 5.304083 & 32.605466 & 15.479559 \\
\hline 11 & 6 & 0 & -2.121256 & 30.072172 & 21.181330 \\
\hline 12 & 1 & 0 & -3.089247 & 29.885271 & 20.721340 \\
\hline 13 & 7 & 0 & 6.644409 & 31.850696 & 13.850325 \\
\hline 14 & 16 & 0 & 7.269231 & 28.574891 & 13.081954 \\
\hline 15 & 6 & 0 & -0.976350 & 30.035234 & 20.379270 \\
\hline 16 & 16 & 0 & 6.059842 & 29.121161 & 18.263790 \\
\hline 17 & 7 & 0 & 8.540667 & 31.319661 & 17.395094 \\
\hline 18 & 6 & 0 & 0.283360 & 30.271242 & 20.971739 \\
\hline 19 & 16 & 0 & 11.644775 & 33.570712 & 16.222218 \\
\hline 20 & 7 & 0 & 10.002877 & 31.068290 & 15.716151 \\
\hline 21 & 6 & 0 & 1.709912 & 30.773337 & 23.018091 \\
\hline 22 & 1 & 0 & 2.376185 & 29.911857 & 22.901140 \\
\hline 23 & 1 & 0 & 2.224451 & 31.634507 & 22.578944 \\
\hline 24 & 1 & 0 & 1.586491 & 30.962708 & 24.087668 \\
\hline 25 & 16 & 0 & 13.979004 & 32.236722 & 13.929400 \\
\hline 26 & 7 & 0 & 14.211691 & 34.744911 & 15.411425 \\
\hline 27 & 6 & 0 & -3.310843 & 30.406046 & 23.389996 \\
\hline 28 & 1 & 0 & -4.081601 & 29.726584 & 23.013505 \\
\hline 29 & 1 & 0 & -3.109985 & 30.146824 & 24.433769 \\
\hline 30 & 1 & 0 & -3.738064 & 31.416731 & 23.382305 \\
\hline 31 & 16 & 0 & 13.565624 & 28.477642 & 11.577247 \\
\hline 32 & 7 & 0 & 9.147554 & 26.624807 & 11.949430 \\
\hline
\end{tabular}




\begin{tabular}{|c|c|c|c|c|c|}
\hline 33 & 6 & 0 & -1.100567 & 29.732782 & 18.903574 \\
\hline 34 & 1 & 0 & -0.732748 & 30.564141 & 18.293020 \\
\hline 35 & 1 & 0 & -0.516894 & 28.848870 & 18.624740 \\
\hline 36 & 1 & 0 & -2.143201 & 29.549560 & 18.630866 \\
\hline 37 & 6 & 0 & 1.534280 & 30.229644 & 20.136118 \\
\hline 38 & 6 & 0 & 1.935808 & 31.390811 & 19.498910 \\
\hline 39 & 6 & 0 & 3.042124 & 31.632701 & 18.618470 \\
\hline 40 & 1 & 0 & 3.734650 & 30.833950 & 18.392783 \\
\hline 41 & 6 & 0 & 3.106720 & 32.905788 & 18.119279 \\
\hline 42 & 1 & 0 & 3.862890 & 33.231283 & 17.410016 \\
\hline 43 & 6 & 0 & 2.061180 & 33.769625 & 18.571885 \\
\hline 44 & 6 & 0 & 1.833179 & 35.094232 & 18.238667 \\
\hline 45 & 6 & 0 & 0.747186 & 35.908336 & 18.680835 \\
\hline 46 & 1 & 0 & -0.018430 & 35.533952 & 19.351298 \\
\hline 47 & 6 & 0 & 0.765881 & 37.178444 & 18.181758 \\
\hline 48 & 1 & 0 & 0.022753 & 37.933246 & 18.405822 \\
\hline 49 & 6 & 0 & 1.877575 & 37.466713 & 17.318711 \\
\hline 50 & 6 & 0 & 2.123560 & 38.694974 & 16.723940 \\
\hline 51 & 6 & 0 & 1.136431 & 39.807250 & 16.957583 \\
\hline 52 & 6 & 0 & 1.293984 & 40.683735 & 18.052038 \\
\hline 53 & 6 & 0 & 0.362795 & 41.710425 & 18.241126 \\
\hline 54 & 1 & 0 & 0.487623 & 42.381029 & 19.088726 \\
\hline 55 & 6 & 0 & -0.718585 & 41.896480 & 17.375495 \\
\hline 56 & 6 & 0 & -0.856807 & 41.016289 & 16.299166 \\
\hline 57 & 1 & 0 & -1.694133 & 41.140258 & 15.615850 \\
\hline 58 & 6 & 0 & 0.050902 & 39.976257 & 16.071941 \\
\hline 59 & 6 & 0 & 2.446880 & 40.527713 & 19.017856 \\
\hline 60 & 1 & 0 & 2.422775 & 41.309794 & 19.781268 \\
\hline 61 & 1 & 0 & 2.419584 & 39.557819 & 19.525947 \\
\hline 62 & 1 & 0 & 3.411596 & 40.585572 & 18.502651 \\
\hline 63 & 6 & 0 & -1.692708 & 43.032355 & 17.582389 \\
\hline 64 & 1 & 0 & -1.822958 & 43.260248 & 18.644746 \\
\hline 65 & 1 & 0 & -2.675530 & 42.798204 & 17.162755 \\
\hline 66 & 1 & 0 & -1.341335 & 43.950549 & 17.095002 \\
\hline 67 & 6 & 0 & -0.144691 & 39.050453 & 14.892949 \\
\hline 68 & 1 & 0 & -1.009360 & 39.354415 & 14.297016 \\
\hline 69 & 1 & 0 & 0.733140 & 39.043510 & 14.238416 \\
\hline 70 & 1 & 0 & -0.303170 & 38.015703 & 15.215272 \\
\hline 71 & 6 & 0 & 3.267292 & 38.980798 & 15.906396 \\
\hline 72 & 6 & 0 & 3.580958 & 40.311222 & 15.375931 \\
\hline 73 & 1 & 0 & 2.980699 & 41.200133 & 15.509791 \\
\hline 74 & 6 & 0 & 4.755927 & 40.185939 & 14.711410 \\
\hline 75 & 1 & 0 & 5.309159 & 40.949652 & 14.183242 \\
\hline 76 & 6 & 0 & 5.142210 & 38.780738 & 14.823698 \\
\hline
\end{tabular}




\begin{tabular}{|c|c|c|c|c|c|}
\hline 77 & 6 & 0 & 6.284521 & 38.224303 & 14.263617 \\
\hline 78 & 6 & 0 & 7.251817 & 39.142500 & 13.569336 \\
\hline 79 & 6 & 0 & 7.226812 & 39.268689 & 12.162546 \\
\hline 80 & 6 & 0 & 8.119456 & 40.149525 & 11.545648 \\
\hline 81 & 1 & 0 & 8.090784 & 40.250107 & 10.462780 \\
\hline 82 & 6 & 0 & 9.038275 & 40.907460 & 12.278417 \\
\hline 83 & 6 & 0 & 9.041411 & 40.769193 & 13.668005 \\
\hline 84 & 1 & 0 & 9.739023 & 41.358862 & 14.258805 \\
\hline 85 & 6 & 0 & 8.166515 & 39.899120 & 14.330121 \\
\hline 86 & 6 & 0 & 8.205597 & 39.799308 & 15.838546 \\
\hline 87 & 1 & 0 & 9.108967 & 40.265593 & 16.238829 \\
\hline 88 & 1 & 0 & 7.339542 & 40.292918 & 16.293331 \\
\hline 89 & 1 & 0 & 8.187480 & 38.757941 & 16.173374 \\
\hline 90 & 6 & 0 & 10.009507 & 41.830928 & 11.581829 \\
\hline 91 & 1 & 0 & 9.528484 & 42.372298 & 10.760828 \\
\hline 92 & 1 & 0 & 10.848796 & 41.271454 & 11.150509 \\
\hline 93 & 1 & 0 & 10.428209 & 42.567620 & 12.273350 \\
\hline 94 & 6 & 0 & 6.241476 & 38.489048 & 11.321818 \\
\hline 95 & 1 & 0 & 6.291299 & 38.800861 & 10.275385 \\
\hline 96 & 1 & 0 & 6.440889 & 37.412845 & 11.363112 \\
\hline 97 & 1 & 0 & 5.214617 & 38.633693 & 11.672466 \\
\hline 98 & 6 & 0 & 6.611800 & 36.826385 & 14.281091 \\
\hline 99 & 6 & 0 & 7.790442 & 36.260124 & 13.788778 \\
\hline 100 & 1 & 0 & 8.601049 & 36.862986 & 13.398769 \\
\hline 101 & 6 & 0 & 7.822290 & 34.856705 & 13.850348 \\
\hline 102 & 1 & 0 & 8.680835 & 34.271120 & 13.548065 \\
\hline 103 & 6 & 0 & 6.661402 & 34.314663 & 14.387812 \\
\hline 104 & 6 & 0 & 6.245797 & 32.945090 & 14.590963 \\
\hline 105 & 6 & 0 & 5.896744 & 30.765738 & 14.297038 \\
\hline 106 & 6 & 0 & 5.086284 & 31.283055 & 15.304614 \\
\hline 107 & 1 & 0 & 4.383689 & 30.733621 & 15.913893 \\
\hline 108 & 6 & 0 & 7.580463 & 31.855180 & 12.731946 \\
\hline 109 & 1 & 0 & 7.304076 & 31.070631 & 12.028109 \\
\hline 110 & 1 & 0 & 7.524116 & 32.818375 & 12.224439 \\
\hline 111 & 1 & 0 & 8.604031 & 31.683056 & 13.078505 \\
\hline 112 & 6 & 0 & 5.902210 & 29.408701 & 13.806805 \\
\hline 113 & 6 & 0 & 4.819774 & 28.546174 & 13.935939 \\
\hline 114 & 1 & 0 & 3.866839 & 28.860653 & 14.343627 \\
\hline 115 & 6 & 0 & 5.074695 & 27.252365 & 13.464323 \\
\hline 116 & 1 & 0 & 4.344032 & 26.453651 & 13.472701 \\
\hline 117 & 6 & 0 & 6.358663 & 27.070490 & 12.947401 \\
\hline 118 & 6 & 0 & 6.855515 & 25.860278 & 12.362822 \\
\hline 119 & 6 & 0 & 5.888229 & 24.709744 & 12.292173 \\
\hline 120 & 6 & 0 & 5.845491 & 23.767257 & 13.340760 \\
\hline
\end{tabular}




\begin{tabular}{|c|c|c|c|c|c|}
\hline 121 & 6 & 0 & 4.929687 & 22.711793 & 13.265327 \\
\hline 122 & 1 & 0 & 4.898121 & 21.987378 & 14.076337 \\
\hline 123 & 6 & 0 & 4.061602 & 22.561067 & 12.180977 \\
\hline 124 & 6 & 0 & 4.121714 & 23.508229 & 11.154871 \\
\hline 125 & 1 & 0 & 3.451908 & 23.412320 & 10.303059 \\
\hline 126 & 6 & 0 & 5.017771 & 24.581445 & 11.189101 \\
\hline 127 & 6 & 0 & 5.044871 & 25.576221 & 10.051037 \\
\hline 128 & 1 & 0 & 4.307358 & 25.314762 & 9.287946 \\
\hline 129 & 1 & 0 & 4.826699 & 26.591353 & 10.399323 \\
\hline 130 & 1 & 0 & 6.029972 & 25.610471 & 9.574112 \\
\hline 131 & 6 & 0 & 6.773283 & 23.873309 & 14.530200 \\
\hline 132 & 1 & 0 & 6.496695 & 23.154125 & 15.305496 \\
\hline 133 & 1 & 0 & 7.811955 & 23.677705 & 14.241276 \\
\hline 134 & 1 & 0 & 6.751089 & 24.873809 & 14.973397 \\
\hline 135 & 6 & 0 & 3.105008 & 21.394569 & 12.105264 \\
\hline 136 & 1 & 0 & 2.180068 & 21.667652 & 11.588168 \\
\hline 137 & 1 & 0 & 3.547216 & 20.554310 & 11.555392 \\
\hline 138 & 1 & 0 & 2.841120 & 21.027349 & 13.101435 \\
\hline 139 & 6 & 0 & 8.142440 & 25.677983 & 11.876913 \\
\hline 140 & 6 & 0 & 8.621576 & 24.477727 & 11.200395 \\
\hline 141 & 1 & 0 & 8.040589 & 23.586439 & 11.009409 \\
\hline 142 & 6 & 0 & 9.909380 & 24.729005 & 10.856788 \\
\hline 143 & 1 & 0 & 10.598239 & 24.080826 & 10.334082 \\
\hline 144 & 6 & 0 & 10.214881 & 26.075666 & 11.353437 \\
\hline 145 & 6 & 0 & 11.523588 & 26.671106 & 11.217930 \\
\hline 146 & 6 & 0 & 12.538144 & 25.919899 & 10.398679 \\
\hline 147 & 6 & 0 & 13.394932 & 24.980506 & 11.015600 \\
\hline 148 & 6 & 0 & 14.327543 & 24.295814 & 10.232429 \\
\hline 149 & 1 & 0 & 14.984857 & 23.573606 & 10.712274 \\
\hline 150 & 6 & 0 & 14.438355 & 24.512249 & 8.854872 \\
\hline 151 & 6 & 0 & 13.580448 & 25.442182 & 8.265350 \\
\hline 152 & 1 & 0 & 13.649040 & 25.624776 & 7.195276 \\
\hline 153 & 6 & 0 & 12.629814 & 26.149896 & 9.010506 \\
\hline 154 & 6 & 0 & 13.308890 & 24.702502 & 12.499001 \\
\hline 155 & 1 & 0 & 14.045009 & 23.951194 & 12.796819 \\
\hline 156 & 1 & 0 & 13.492072 & 25.607029 & 13.088253 \\
\hline 157 & 1 & 0 & 12.316482 & 24.333746 & 12.780621 \\
\hline 158 & 6 & 0 & 15.452464 & 23.750737 & 8.034071 \\
\hline 159 & 1 & 0 & 15.480539 & 24.108380 & 7.001202 \\
\hline 160 & 1 & 0 & 16.460385 & 23.850888 & 8.451878 \\
\hline 161 & 1 & 0 & 15.221546 & 22.679065 & 8.008793 \\
\hline 162 & 6 & 0 & 11.720832 & 27.142343 & 8.322181 \\
\hline 163 & 1 & 0 & 11.931247 & 27.184034 & 7.250242 \\
\hline 164 & 1 & 0 & 10.666056 & 26.876464 & 8.450524 \\
\hline
\end{tabular}




\begin{tabular}{|c|c|c|c|c|c|}
\hline 165 & 1 & 0 & 11.846230 & 28.150605 & 8.730803 \\
\hline 166 & 6 & 0 & 11.911368 & 27.863339 & 11.804350 \\
\hline 167 & 6 & 0 & 11.188175 & 28.757304 & 12.661832 \\
\hline 168 & 1 & 0 & 10.157055 & 28.547211 & 12.909262 \\
\hline 169 & 6 & 0 & 11.911816 & 29.830360 & 13.108544 \\
\hline 170 & 1 & 0 & 11.516125 & 30.575741 & 13.793252 \\
\hline 171 & 6 & 0 & 13.258359 & 29.872975 & 12.629539 \\
\hline 172 & 6 & 0 & 14.240531 & 30.812201 & 12.896019 \\
\hline 173 & 6 & 0 & 15.581036 & 30.801455 & 12.405547 \\
\hline 174 & 1 & 0 & 15.950988 & 30.015518 & 11.756497 \\
\hline 175 & 6 & 0 & 16.335093 & 31.858608 & 12.825256 \\
\hline 176 & 1 & 0 & 17.371174 & 32.015657 & 12.553312 \\
\hline 177 & 6 & 0 & 15.642573 & 32.789575 & 13.672305 \\
\hline 178 & 6 & 0 & 16.192780 & 33.953386 & 14.187907 \\
\hline 179 & 6 & 0 & 17.626112 & 34.268869 & 13.852886 \\
\hline 180 & 6 & 0 & 18.659820 & 33.859021 & 14.721622 \\
\hline 181 & 6 & 0 & 19.984024 & 34.174719 & 14.400187 \\
\hline 182 & 1 & 0 & 20.776502 & 33.858420 & 15.075143 \\
\hline 183 & 6 & 0 & 20.315097 & 34.884237 & 13.242481 \\
\hline 184 & 6 & 0 & 19.275876 & 35.285567 & 12.399030 \\
\hline 185 & 1 & 0 & 19.509955 & 35.846483 & 11.496784 \\
\hline 186 & 6 & 0 & 17.937538 & 34.991086 & 12.681419 \\
\hline 187 & 6 & 0 & 18.355050 & 33.093086 & 15.988947 \\
\hline 188 & 1 & 0 & 19.273931 & 32.857443 & 16.532221 \\
\hline 189 & 1 & 0 & 17.703765 & 33.666692 & 16.657120 \\
\hline 190 & 1 & 0 & 17.835789 & 32.152676 & 15.776113 \\
\hline 191 & 6 & 0 & 21.755362 & 35.185389 & 12.900868 \\
\hline 192 & 1 & 0 & 21.841599 & 36.088986 & 12.290134 \\
\hline 193 & 1 & 0 & 22.359942 & 35.326250 & 13.802017 \\
\hline 194 & 1 & 0 & 22.208817 & 34.363915 & 12.331905 \\
\hline 195 & 6 & 0 & 16.850705 & 35.452118 & 11.736845 \\
\hline 196 & 1 & 0 & 17.271338 & 36.037747 & 10.915087 \\
\hline 197 & 1 & 0 & 16.307210 & 34.605135 & 11.304406 \\
\hline 198 & 1 & 0 & 16.108542 & 36.071872 & 12.250883 \\
\hline 199 & 6 & 0 & 15.478141 & 34.894909 & 15.000777 \\
\hline 200 & 6 & 0 & 16.040598 & 36.169877 & 15.456909 \\
\hline 201 & 1 & 0 & 17.044340 & 36.520834 & 15.262774 \\
\hline 202 & 6 & 0 & 15.053645 & 36.793859 & 16.145215 \\
\hline 203 & 1 & 0 & 15.087498 & 37.756329 & 16.635870 \\
\hline 204 & 6 & 0 & 13.902593 & 35.893515 & 16.117425 \\
\hline 205 & 6 & 0 & 12.677417 & 36.155237 & 16.715948 \\
\hline 206 & 6 & 0 & 12.482454 & 37.494195 & 17.372220 \\
\hline 207 & 6 & 0 & 12.635299 & 37.630221 & 18.769622 \\
\hline 208 & 6 & 0 & 12.464374 & 38.890351 & 19.350050 \\
\hline
\end{tabular}




\begin{tabular}{|c|c|c|c|c|c|}
\hline 209 & 1 & 0 & 12.584604 & 38.991088 & 20.426575 \\
\hline 210 & 6 & 0 & 12.150186 & 40.020356 & 18.588851 \\
\hline 211 & 6 & 0 & 12.003457 & 39.861059 & 17.209078 \\
\hline 212 & 1 & 0 & 11.762190 & 40.727393 & 16.596856 \\
\hline 213 & 6 & 0 & 12.163354 & 38.618553 & 16.583662 \\
\hline 214 & 6 & 0 & 12.997730 & 36.446833 & 19.637957 \\
\hline 215 & 1 & 0 & 13.914205 & 35.961445 & 19.286981 \\
\hline 216 & 1 & 0 & 12.212520 & 35.683667 & 19.627954 \\
\hline 217 & 1 & 0 & 13.153046 & 36.757732 & 20.674249 \\
\hline 218 & 6 & 0 & 12.002981 & 41.375744 & 19.239050 \\
\hline 219 & 1 & 0 & 11.422941 & 42.059578 & 18.612808 \\
\hline 220 & 1 & 0 & 11.505106 & 41.301818 & 20.211021 \\
\hline 221 & 1 & 0 & 12.981455 & 41.840803 & 19.412592 \\
\hline 222 & 6 & 0 & 12.005881 & 38.506511 & 15.084048 \\
\hline 223 & 1 & 0 & 11.545706 & 39.406600 & 14.669897 \\
\hline 224 & 1 & 0 & 12.974637 & 38.363831 & 14.592492 \\
\hline 225 & 1 & 0 & 11.382193 & 37.651220 & 14.807243 \\
\hline 226 & 6 & 0 & 11.572722 & 35.239756 & 16.766733 \\
\hline 227 & 6 & 0 & 10.313295 & 35.515255 & 17.305434 \\
\hline 228 & 1 & 0 & 10.049654 & 36.494506 & 17.685024 \\
\hline 229 & 6 & 0 & 9.436269 & 34.417750 & 17.304664 \\
\hline 230 & 1 & 0 & 8.412325 & 34.479696 & 17.649512 \\
\hline 231 & 6 & 0 & 10.007886 & 33.271722 & 16.766296 \\
\hline 232 & 6 & 0 & 9.496719 & 31.928970 & 16.607604 \\
\hline 233 & 6 & 0 & 7.843002 & 31.913846 & 18.529495 \\
\hline 234 & 1 & 0 & 7.597743 & 31.133297 & 19.249041 \\
\hline 235 & 1 & 0 & 8.497174 & 32.642109 & 19.009556 \\
\hline 236 & 1 & 0 & 6.920373 & 32.406307 & 18.207682 \\
\hline 237 & 6 & 0 & 8.454636 & 29.995898 & 16.975912 \\
\hline 238 & 6 & 0 & 9.375572 & 29.891089 & 15.937237 \\
\hline 239 & 1 & 0 & 9.575710 & 29.014881 & 15.338415 \\
\hline 240 & 6 & 0 & 7.640117 & 28.934529 & 17.517730 \\
\hline 241 & 6 & 0 & 7.979540 & 27.590158 & 17.426685 \\
\hline 242 & 1 & 0 & 8.921629 & 27.251572 & 17.013034 \\
\hline 243 & 6 & 0 & 7.003514 & 26.729706 & 17.945899 \\
\hline 244 & 1 & 0 & 7.102731 & 25.652148 & 17.973043 \\
\hline 245 & 6 & 0 & 5.879271 & 27.377980 & 18.459418 \\
\hline 246 & 6 & 0 & 4.760956 & 26.732156 & 19.081943 \\
\hline 247 & 6 & 0 & 4.844319 & 25.235002 & 19.207608 \\
\hline 248 & 6 & 0 & 5.465878 & 24.651346 & 20.331721 \\
\hline 249 & 6 & 0 & 5.535022 & 23.257165 & 20.419860 \\
\hline 250 & 1 & 0 & 6.009046 & 22.810945 & 21.291363 \\
\hline 251 & 6 & 0 & 5.013085 & 22.425738 & 19.425010 \\
\hline 252 & 6 & 0 & 4.400192 & 23.026593 & 18.322375 \\
\hline
\end{tabular}




\begin{tabular}{rrrrrr}
253 & 1 & 0 & 3.978096 & 22.398710 & 17.540634 \\
254 & 6 & 0 & 4.304885 & 24.416639 & 18.192994 \\
255 & 6 & 0 & 6.040192 & 25.505359 & 21.438810 \\
256 & 1 & 0 & 6.477255 & 24.883295 & 22.224115 \\
257 & 1 & 0 & 6.820885 & 26.175947 & 21.064356 \\
258 & 1 & 0 & 5.271607 & 26.138231 & 21.894710 \\
259 & 6 & 0 & 5.131019 & 20.923295 & 19.527372 \\
260 & 1 & 0 & 4.320112 & 20.420623 & 18.991932 \\
261 & 1 & 0 & 6.075300 & 20.568771 & 19.095296 \\
262 & 1 & 0 & 5.107169 & 20.590045 & 20.569272 \\
263 & 6 & 0 & 3.617853 & 25.013841 & 16.985625 \\
264 & 1 & 0 & 3.379432 & 24.240763 & 16.250461 \\
265 & 1 & 0 & 2.684556 & 25.514256 & 17.266352 \\
266 & 1 & 0 & 4.244973 & 25.764940 & 16.494878 \\
267 & 6 & 0 & 3.629210 & 27.378252 & 19.557333 \\
268 & 6 & 0 & 2.531843 & 26.733490 & 20.270512 \\
269 & 1 & 0 & 2.461599 & 25.679554 & 20.500458 \\
270 & 6 & 0 & 1.658524 & 27.721411 & 20.587995 \\
271 & 1 & 0 & 0.724048 & 27.637563 & 21.124389 \\
272 & 6 & 0 & 2.219644 & 28.961947 & 20.039497 \\
\hline-----------------------------------
\end{tabular}

A proposed tub structure of $\mathbf{8}$ upon optimization (Sum of imaginary frequencies $=0$, total energy $=-8878.43929640$ Hartrees)

\begin{tabular}{cccrrr}
$\begin{array}{c}\text { Center } \\
\text { Number }\end{array}$ & $\begin{array}{c}\text { Atomic } \\
\text { Number }\end{array}$ & $\begin{array}{c}\text { Atomic } \\
\text { Type }\end{array}$ & \multicolumn{3}{c}{ Coordinates (Angstroms) } \\
\hline 1 & 6 & 0 & -11.174263 & -3.590805 & 1.489781 \\
2 & 16 & 0 & -9.788846 & -0.530005 & -0.173378 \\
3 & 7 & 0 & -7.823279 & -1.994824 & 3.876865 \\
4 & 6 & 0 & -12.436580 & -4.115609 & 1.197187 \\
5 & 1 & 0 & -12.504052 & -5.123072 & 0.792317 \\
6 & 16 & 0 & -7.032556 & 2.446000 & -2.008355 \\
7 & 7 & 0 & -5.246935 & 4.221059 & -2.904823 \\
8 & 6 & 0 & -13.608681 & -3.385693 & 1.412568 \\
9 & 16 & 0 & -0.768118 & 5.294110 & -2.472248 \\
10 & 7 & 0 & 2.283437 & 5.769177 & -2.882916 \\
11 & 6 & 0 & -13.493123 & -2.097811 & 1.942119 \\
12 & 1 & 0 & -14.393680 & -1.516744 & 2.128978 \\
13 & 7 & 0 & 2.784282 & 5.664261 & -0.700491 \\
14 & 16 & 0 & 5.956171 & 6.012244 & 0.530991 \\
15 & 6 & 0 & -12.251114 & -1.529528 & 2.243662 \\
16 & 16 & 0 & -5.039385 & -2.107089 & 3.364056 \\
& & & & &
\end{tabular}




\begin{tabular}{|c|c|c|c|c|c|}
\hline 17 & 7 & 0 & -1.309015 & -2.587246 & 1.945722 \\
\hline 18 & 6 & 0 & -11.079198 & -2.282645 & 2.013010 \\
\hline 19 & 16 & 0 & -0.071419 & -2.943677 & -1.832685 \\
\hline 20 & 7 & 0 & -1.941170 & -1.490872 & 0.094911 \\
\hline 21 & 6 & 0 & -9.935954 & -4.421133 & 1.240268 \\
\hline 22 & 1 & 0 & -9.383450 & -4.608705 & 2.167176 \\
\hline 23 & 1 & 0 & -9.244623 & -3.914387 & 0.558909 \\
\hline 24 & 1 & 0 & -10.197306 & -5.387449 & 0.800928 \\
\hline 25 & 16 & 0 & 6.394152 & -3.747846 & -1.106733 \\
\hline 26 & 7 & 0 & 4.198192 & -4.928179 & -2.656400 \\
\hline 27 & 6 & 0 & -14.959294 & -3.962808 & 1.060379 \\
\hline 28 & 1 & 0 & -15.748506 & -3.553256 & 1.698004 \\
\hline 29 & 1 & 0 & -14.969302 & -5.051994 & 1.164455 \\
\hline 30 & 1 & 0 & -15.229241 & -3.733605 & 0.021780 \\
\hline 31 & 16 & 0 & 9.773971 & -1.933335 & 1.175360 \\
\hline 32 & 7 & 0 & 9.053038 & 2.732406 & 1.619737 \\
\hline 33 & 6 & 0 & -12.185601 & -0.133269 & 2.820214 \\
\hline 34 & 1 & 0 & -11.690765 & 0.560928 & 2.132415 \\
\hline 35 & 1 & 0 & -11.621000 & -0.109228 & 3.758196 \\
\hline 36 & 1 & 0 & -13.189428 & 0.251253 & 3.019156 \\
\hline 37 & 6 & 0 & -9.730077 & -1.709734 & 2.337342 \\
\hline 38 & 6 & 0 & -9.107843 & -0.860951 & 1.441107 \\
\hline 39 & 6 & 0 & -7.916097 & -0.079878 & 1.622106 \\
\hline 40 & 1 & 0 & -7.352969 & -0.151905 & 2.541286 \\
\hline 41 & 6 & 0 & -7.596204 & 0.714555 & 0.561498 \\
\hline 42 & 1 & 0 & -6.736195 & 1.375262 & 0.543283 \\
\hline 43 & 6 & 0 & -8.481277 & 0.608285 & -0.558506 \\
\hline 44 & 6 & 0 & -8.338490 & 1.257562 & -1.770814 \\
\hline 45 & 6 & 0 & -9.094827 & 1.101977 & -2.972365 \\
\hline 46 & 1 & 0 & -9.944141 & 0.430798 & -3.043031 \\
\hline 47 & 6 & 0 & -8.615894 & 1.838315 & -4.020660 \\
\hline 48 & 1 & 0 & -9.040004 & 1.828147 & -5.017610 \\
\hline 49 & 6 & 0 & -7.455259 & 2.633688 & -3.720002 \\
\hline 50 & 6 & 0 & -6.727212 & 3.398034 & -4.620403 \\
\hline 51 & 6 & 0 & -7.124829 & 3.436931 & -6.066982 \\
\hline 52 & 6 & 0 & -6.672766 & 2.437050 & -6.954612 \\
\hline 53 & 6 & 0 & -7.040182 & 2.508559 & -8.302819 \\
\hline 54 & 1 & 0 & -6.684578 & 1.739050 & -8.984579 \\
\hline 55 & 6 & 0 & -7.844552 & 3.538622 & -8.796667 \\
\hline 56 & 6 & 0 & -8.276812 & 4.521523 & -7.901816 \\
\hline 57 & 1 & 0 & -8.898234 & 5.336807 & -8.266319 \\
\hline 58 & 6 & 0 & -7.931330 & 4.491171 & -6.547140 \\
\hline 59 & 6 & 0 & -5.795579 & 1.303346 & -6.473225 \\
\hline 60 & 1 & 0 & -5.481002 & 0.672736 & -7.309043 \\
\hline
\end{tabular}




\begin{tabular}{|c|c|c|c|c|c|}
\hline 61 & 1 & 0 & -6.317816 & 0.668160 & -5.749685 \\
\hline 62 & 1 & 0 & -4.896605 & 1.675745 & -5.971345 \\
\hline 63 & 6 & 0 & -8.256201 & 3.576379 & -10.249611 \\
\hline 64 & 1 & 0 & -7.526154 & 3.067507 & -10.885974 \\
\hline 65 & 1 & 0 & -9.222674 & 3.079387 & -10.401644 \\
\hline 66 & 1 & 0 & -8.361818 & 4.604393 & -10.609602 \\
\hline 67 & 6 & 0 & -8.420770 & 5.580860 & -5.620438 \\
\hline 68 & 1 & 0 & -9.058680 & 6.289787 & -6.154809 \\
\hline 69 & 1 & 0 & -7.586633 & 6.140456 & -5.183072 \\
\hline 70 & 1 & 0 & -8.994959 & 5.169366 & -4.783744 \\
\hline 71 & 6 & 0 & -5.563899 & 4.126126 & -4.192998 \\
\hline 72 & 6 & 0 & -4.557371 & 4.786398 & -5.031714 \\
\hline 73 & 1 & 0 & -4.589639 & 4.878193 & -6.109219 \\
\hline 74 & 6 & 0 & -3.596950 & 5.244041 & -4.185311 \\
\hline 75 & 1 & 0 & -2.709530 & 5.804248 & -4.444859 \\
\hline 76 & 6 & 0 & -4.036137 & 4.887334 & -2.833356 \\
\hline 77 & 6 & 0 & -3.461566 & 5.144507 & -1.600307 \\
\hline 78 & 6 & 0 & -4.256125 & 4.861623 & -0.358620 \\
\hline 79 & 6 & 0 & -3.938019 & 3.733850 & 0.431792 \\
\hline 80 & 6 & 0 & -4.684348 & 3.486769 & 1.587526 \\
\hline 81 & 1 & 0 & -4.439507 & 2.615555 & 2.191896 \\
\hline 82 & 6 & 0 & -5.729972 & 4.325129 & 1.989035 \\
\hline 83 & 6 & 0 & -6.019956 & 5.436196 & 1.195064 \\
\hline 84 & 1 & 0 & -6.826524 & 6.104010 & 1.489506 \\
\hline 85 & 6 & 0 & -5.311328 & 5.715605 & 0.021377 \\
\hline 86 & 6 & 0 & -5.704709 & 6.909356 & -0.817279 \\
\hline 87 & 1 & 0 & -6.374435 & 7.571181 & -0.261429 \\
\hline 88 & 1 & 0 & -6.224919 & 6.582224 & -1.723961 \\
\hline 89 & 1 & 0 & -4.836070 & 7.493355 & -1.137157 \\
\hline 90 & 6 & 0 & -6.539096 & 4.015249 & 3.226347 \\
\hline 91 & 1 & 0 & -5.907598 & 3.628994 & 4.033017 \\
\hline 92 & 1 & 0 & -7.059650 & 4.902785 & 3.597514 \\
\hline 93 & 1 & 0 & -7.299512 & 3.252239 & 3.018313 \\
\hline 94 & 6 & 0 & -2.841165 & 2.773877 & 0.031711 \\
\hline 95 & 1 & 0 & -2.833237 & 1.894175 & 0.680250 \\
\hline 96 & 1 & 0 & -1.851129 & 3.238721 & 0.090657 \\
\hline 97 & 1 & 0 & -2.972092 & 2.428669 & -0.998578 \\
\hline 98 & 6 & 0 & -2.113626 & 5.616432 & -1.383554 \\
\hline 99 & 6 & 0 & -1.648934 & 6.231702 & -0.227677 \\
\hline 100 & 1 & 0 & -2.313012 & 6.512533 & 0.580159 \\
\hline 101 & 6 & 0 & -0.255572 & 6.439496 & -0.209653 \\
\hline 102 & 1 & 0 & 0.260764 & 6.932897 & 0.604028 \\
\hline 103 & 6 & 0 & 0.384643 & 5.960514 & -1.340811 \\
\hline 104 & 6 & 0 & 1.794711 & 5.844862 & -1.647120 \\
\hline
\end{tabular}




\begin{tabular}{|c|c|c|c|c|c|}
\hline 105 & 6 & 0 & 3.967612 & 5.451210 & -1.401613 \\
\hline 106 & 6 & 0 & 3.613028 & 5.529689 & -2.739765 \\
\hline 107 & 1 & 0 & 4.275035 & 5.445612 & -3.590497 \\
\hline 108 & 6 & 0 & 2.604507 & 5.469785 & 0.733353 \\
\hline 109 & 1 & 0 & 3.367438 & 4.782216 & 1.097564 \\
\hline 110 & 1 & 0 & 1.619382 & 5.040295 & 0.916322 \\
\hline 111 & 1 & 0 & 2.693049 & 6.411676 & 1.283692 \\
\hline 112 & 6 & 0 & 5.239967 & 5.114829 & -0.792049 \\
\hline 113 & 6 & 0 & 6.062181 & 4.064436 & -1.162418 \\
\hline 114 & 1 & 0 & 5.800475 & 3.383744 & -1.964418 \\
\hline 115 & 6 & 0 & 7.221358 & 3.939999 & -0.371639 \\
\hline 116 & 1 & 0 & 7.966511 & 3.165394 & -0.484318 \\
\hline 117 & 6 & 0 & 7.324345 & 4.900156 & 0.627407 \\
\hline 118 & 6 & 0 & 8.325231 & 5.070917 & 1.653505 \\
\hline 119 & 6 & 0 & 8.429273 & 6.412892 & 2.317104 \\
\hline 120 & 6 & 0 & 7.880383 & 6.613874 & 3.601445 \\
\hline 121 & 6 & 0 & 7.977000 & 7.881403 & 4.187969 \\
\hline 122 & 1 & 0 & 7.543287 & 8.035395 & 5.173443 \\
\hline 123 & 6 & 0 & 8.609357 & 8.948066 & 3.547257 \\
\hline 124 & 6 & 0 & 9.150923 & 8.724532 & 2.277044 \\
\hline 125 & 1 & 0 & 9.650368 & 9.541093 & 1.760168 \\
\hline 126 & 6 & 0 & 9.070932 & 7.480710 & 1.647559 \\
\hline 127 & 6 & 0 & 9.689940 & 7.289148 & 0.281937 \\
\hline 128 & 1 & 0 & 10.193021 & 8.201620 & -0.047872 \\
\hline 129 & 1 & 0 & 8.938120 & 7.028622 & -0.470394 \\
\hline 130 & 1 & 0 & 10.426015 & 6.478215 & 0.287974 \\
\hline 131 & 6 & 0 & 7.191982 & 5.496656 & 4.354488 \\
\hline 132 & 1 & 0 & 6.648088 & 5.891287 & 5.216914 \\
\hline 133 & 1 & 0 & 7.912463 & 4.757891 & 4.721833 \\
\hline 134 & 1 & 0 & 6.481813 & 4.956694 & 3.721115 \\
\hline 135 & 6 & 0 & 8.726801 & 10.300304 & 4.209426 \\
\hline 136 & 1 & 0 & 8.515532 & 11.110667 & 3.504185 \\
\hline 137 & 1 & 0 & 9.740199 & 10.465921 & 4.595361 \\
\hline 138 & 1 & 0 & 8.035399 & 10.395435 & 5.051284 \\
\hline 139 & 6 & 0 & 9.166117 & 4.043198 & 2.053140 \\
\hline 140 & 6 & 0 & 10.292886 & 4.168942 & 2.976283 \\
\hline 141 & 1 & 0 & 10.613651 & 5.082269 & 3.458086 \\
\hline 142 & 6 & 0 & 10.849084 & 2.933924 & 3.065133 \\
\hline 143 & 1 & 0 & 11.706524 & 2.629808 & 3.649396 \\
\hline 144 & 6 & 0 & 10.033202 & 2.053927 & 2.216549 \\
\hline 145 & 6 & 0 & 10.263048 & 0.632833 & 2.068520 \\
\hline 146 & 6 & 0 & 11.549138 & 0.075225 & 2.609865 \\
\hline 147 & 6 & 0 & 11.592682 & -0.483927 & 3.905338 \\
\hline 148 & 6 & 0 & 12.805945 & -0.984857 & 4.388339 \\
\hline
\end{tabular}




\begin{tabular}{|c|c|c|c|c|c|}
\hline 149 & 1 & 0 & 12.836122 & -1.408894 & 5.389743 \\
\hline 150 & 6 & 0 & 13.975938 & -0.951656 & 3.625075 \\
\hline 151 & 6 & 0 & 13.912272 & -0.387870 & 2.347903 \\
\hline 152 & 1 & 0 & 14.813394 & -0.344905 & 1.740049 \\
\hline 153 & 6 & 0 & 12.722187 & 0.127401 & 1.825598 \\
\hline 154 & 6 & 0 & 10.358035 & -0.534286 & 4.776328 \\
\hline 155 & 1 & 0 & 10.593331 & -0.949183 & 5.759985 \\
\hline 156 & 1 & 0 & 9.575557 & -1.155184 & 4.327175 \\
\hline 157 & 1 & 0 & 9.928534 & 0.462575 & 4.922640 \\
\hline 158 & 6 & 0 & 15.266629 & -1.530122 & 4.155144 \\
\hline 159 & 1 & 0 & 16.137055 & -1.002324 & 3.753759 \\
\hline 160 & 1 & 0 & 15.371221 & -2.586367 & 3.876823 \\
\hline 161 & 1 & 0 & 15.310892 & -1.476821 & 5.247048 \\
\hline 162 & 6 & 0 & 12.705729 & 0.732683 & 0.440631 \\
\hline 163 & 1 & 0 & 13.688307 & 0.652196 & -0.031790 \\
\hline 164 & 1 & 0 & 12.430438 & 1.792606 & 0.469077 \\
\hline 165 & 1 & 0 & 11.976674 & 0.232769 & -0.205457 \\
\hline 166 & 6 & 0 & 9.370685 & -0.218333 & 1.441747 \\
\hline 167 & 6 & 0 & 8.056564 & 0.045261 & 0.921792 \\
\hline 168 & 1 & 0 & 7.636030 & 1.038210 & 0.999275 \\
\hline 169 & 6 & 0 & 7.451780 & -1.033624 & 0.343292 \\
\hline 170 & 1 & 0 & 6.465005 & -1.001040 & -0.106068 \\
\hline 171 & 6 & 0 & 8.233345 & -2.230971 & 0.340215 \\
\hline 172 & 6 & 0 & 7.933305 & -3.437878 & -0.261856 \\
\hline 173 & 6 & 0 & 8.790400 & -4.576005 & -0.377372 \\
\hline 174 & 1 & 0 & 9.772044 & -4.604297 & 0.082611 \\
\hline 175 & 6 & 0 & 8.286662 & -5.577021 & -1.154950 \\
\hline 176 & 1 & 0 & 8.811649 & -6.496313 & -1.382622 \\
\hline 177 & 6 & 0 & 6.990954 & -5.308878 & -1.718694 \\
\hline 178 & 6 & 0 & 6.361918 & -6.077719 & -2.678711 \\
\hline 179 & 6 & 0 & 7.067288 & -7.291034 & -3.217828 \\
\hline 180 & 6 & 0 & 6.704816 & -8.573833 & -2.751515 \\
\hline 181 & 6 & 0 & 7.356081 & -9.696011 & -3.271181 \\
\hline 182 & 1 & 0 & 7.074220 & -10.681419 & -2.906262 \\
\hline 183 & 6 & 0 & 8.353398 & -9.587089 & -4.245111 \\
\hline 184 & 6 & 0 & 8.688642 & -8.310196 & -4.701170 \\
\hline 185 & 1 & 0 & 9.452799 & -8.203766 & -5.467991 \\
\hline 186 & 6 & 0 & 8.066607 & -7.158227 & -4.205650 \\
\hline 187 & 6 & 0 & 5.630954 & -8.746110 & -1.701403 \\
\hline 188 & 1 & 0 & 5.519764 & -9.798403 & -1.426859 \\
\hline 189 & 1 & 0 & 4.660010 & -8.384958 & -2.057236 \\
\hline 190 & 1 & 0 & 5.862010 & -8.179644 & -0.793165 \\
\hline 191 & 6 & 0 & 9.060131 & -10.814153 & -4.770773 \\
\hline 192 & 1 & 0 & 9.516652 & -10.625960 & -5.746729 \\
\hline
\end{tabular}




\begin{tabular}{|c|c|c|c|c|c|}
\hline 193 & 1 & 0 & 8.371921 & -11.658822 & -4.875579 \\
\hline 194 & 1 & 0 & 9.860570 & -11.132887 & -4.091334 \\
\hline 195 & 6 & 0 & 8.463761 & -5.802602 & -4.745913 \\
\hline 196 & 1 & 0 & 9.144482 & -5.907874 & -5.594787 \\
\hline 197 & 1 & 0 & 8.967439 & -5.195713 & -3.985617 \\
\hline 198 & 1 & 0 & 7.591299 & -5.230859 & -5.077812 \\
\hline 199 & 6 & 0 & 5.077678 & -5.744408 & -3.242160 \\
\hline 200 & 6 & 0 & 4.603737 & -6.207304 & -4.546782 \\
\hline 201 & 1 & 0 & 5.130334 & -6.881689 & -5.207637 \\
\hline 202 & 6 & 0 & 3.412018 & -5.590221 & -4.751521 \\
\hline 203 & 1 & 0 & 2.758946 & -5.669436 & -5.609189 \\
\hline 204 & 6 & 0 & 3.135895 & -4.817373 & -3.541645 \\
\hline 205 & 6 & 0 & 1.932833 & -4.169155 & -3.288282 \\
\hline 206 & 6 & 0 & 0.936886 & -4.055060 & -4.410924 \\
\hline 207 & 6 & 0 & -0.030184 & -5.062766 & -4.615825 \\
\hline 208 & 6 & 0 & -0.943784 & -4.918610 & -5.664441 \\
\hline 209 & 1 & 0 & -1.686134 & -5.697964 & -5.821335 \\
\hline 210 & 6 & 0 & -0.930408 & -3.806872 & -6.510828 \\
\hline 211 & 6 & 0 & 0.041397 & -2.826861 & -6.293584 \\
\hline 212 & 1 & 0 & 0.074499 & -1.956151 & -6.944483 \\
\hline 213 & 6 & 0 & 0.977570 & -2.928163 & -5.259997 \\
\hline 214 & 6 & 0 & -0.078770 & -6.294470 & -3.740848 \\
\hline 215 & 1 & 0 & 0.836006 & -6.889298 & -3.836845 \\
\hline 216 & 1 & 0 & -0.179136 & -6.032708 & -2.682588 \\
\hline 217 & 1 & 0 & -0.925595 & -6.930002 & -4.012205 \\
\hline 218 & 6 & 0 & -1.954141 & -3.655693 & -7.610709 \\
\hline 219 & 1 & 0 & -2.842384 & -3.121194 & -7.251514 \\
\hline 220 & 1 & 0 & -2.289112 & -4.628538 & -7.982778 \\
\hline 221 & 1 & 0 & -1.553511 & -3.087768 & -8.455699 \\
\hline 222 & 6 & 0 & 2.014857 & -1.844096 & -5.074706 \\
\hline 223 & 1 & 0 & 1.866998 & -1.037835 & -5.797527 \\
\hline 224 & 1 & 0 & 3.029724 & -2.234844 & -5.203993 \\
\hline 225 & 1 & 0 & 1.967863 & -1.408589 & -4.071042 \\
\hline 226 & 6 & 0 & 1.535843 & -3.648911 & -2.007200 \\
\hline 227 & 6 & 0 & 2.179951 & -3.688409 & -0.770750 \\
\hline 228 & 1 & 0 & 3.182897 & -4.081616 & -0.670471 \\
\hline 229 & 6 & 0 & 1.393162 & -3.195153 & 0.286173 \\
\hline 230 & 1 & 0 & 1.747376 & -3.138743 & 1.308438 \\
\hline 231 & 6 & 0 & 0.132094 & -2.763799 & -0.111659 \\
\hline 232 & 6 & 0 & -0.996928 & -2.258227 & 0.639989 \\
\hline 233 & 6 & 0 & -0.621753 & -3.561126 & 2.782425 \\
\hline 234 & 1 & 0 & -1.351568 & -4.062479 & 3.418660 \\
\hline 235 & 1 & 0 & 0.133971 & -3.086798 & 3.417560 \\
\hline 236 & 1 & 0 & -0.134696 & -4.300297 & 2.145775 \\
\hline
\end{tabular}




\begin{tabular}{|c|c|c|c|c|c|}
\hline 237 & 6 & 0 & -2.537219 & -1.990075 & 2.224806 \\
\hline 238 & 6 & 0 & -2.882468 & -1.323376 & 1.053172 \\
\hline 239 & 1 & 0 & -3.765811 & -0.721985 & 0.886567 \\
\hline 240 & 6 & 0 & -3.285988 & -2.135434 & 3.450876 \\
\hline 241 & 6 & 0 & -2.856914 & -2.313714 & 4.759004 \\
\hline 242 & 1 & 0 & -1.814328 & -2.327340 & 5.052820 \\
\hline 243 & 6 & 0 & -3.917172 & -2.434426 & 5.676589 \\
\hline 244 & 1 & 0 & -3.777694 & -2.584503 & 6.740170 \\
\hline 245 & 6 & 0 & -5.186305 & -2.347121 & 5.105316 \\
\hline 246 & 6 & 0 & -6.434864 & -2.525884 & 5.797378 \\
\hline 247 & 6 & 0 & -6.364634 & -2.901078 & 7.250789 \\
\hline 248 & 6 & 0 & -6.268919 & -4.257311 & 7.629113 \\
\hline 249 & 6 & 0 & -6.213332 & -4.578301 & 8.989022 \\
\hline 250 & 1 & 0 & -6.147670 & -5.625237 & 9.277239 \\
\hline 251 & 6 & 0 & -6.242394 & -3.596401 & 9.983456 \\
\hline 252 & 6 & 0 & -6.338781 & -2.260880 & 9.584888 \\
\hline 253 & 1 & 0 & -6.369691 & -1.480703 & 10.342224 \\
\hline 254 & 6 & 0 & -6.401179 & -1.892833 & 8.236552 \\
\hline 255 & 6 & 0 & -6.244553 & -5.358355 & 6.593652 \\
\hline 256 & 1 & 0 & -6.142387 & -6.337521 & 7.068638 \\
\hline 257 & 1 & 0 & -5.412572 & -5.232301 & 5.892854 \\
\hline 258 & 1 & 0 & -7.162344 & -5.365271 & 5.996179 \\
\hline 259 & 6 & 0 & -6.145580 & -3.968145 & 11.444270 \\
\hline 260 & 1 & 0 & -6.618301 & -3.215012 & 12.081435 \\
\hline 261 & 1 & 0 & -5.099185 & -4.054164 & 11.763230 \\
\hline 262 & 1 & 0 & -6.624373 & -4.931402 & 11.644995 \\
\hline 263 & 6 & 0 & -6.515532 & -0.433064 & 7.859246 \\
\hline 264 & 1 & 0 & -6.451387 & 0.204217 & 8.745021 \\
\hline 265 & 1 & 0 & -7.466877 & -0.222709 & 7.359156 \\
\hline 266 & 1 & 0 & -5.722091 & -0.133394 & 7.166772 \\
\hline 267 & 6 & 0 & -7.673381 & -2.405108 & 5.187491 \\
\hline 268 & 6 & 0 & -8.972181 & -2.750086 & 5.757331 \\
\hline 269 & 1 & 0 & -9.148086 & -3.097648 & 6.766109 \\
\hline 270 & 6 & 0 & -9.877697 & -2.579627 & 4.758084 \\
\hline 271 & 1 & 0 & -10.945519 & -2.747930 & 4.789082 \\
\hline 272 & 6 & 0 & -9.127009 & -2.078398 & 3.600952 \\
\hline
\end{tabular}


9 (The X-ray crystallographic structure of 9 were used for optimization, sum of imaginary frequencies $=0$, total energy $=-8878.46592170$ Hartrees)

\begin{tabular}{|c|c|c|c|c|c|}
\hline \multirow{2}{*}{$\begin{array}{l}\text { Center } \\
\text { Number }\end{array}$} & \multirow{2}{*}{$\begin{array}{l}\text { Atomic } \\
\text { Number }\end{array}$} & \multirow{2}{*}{$\begin{array}{l}\text { Atomic } \\
\text { Type }\end{array}$} & \multicolumn{3}{|c|}{ Coordinates (Angstroms) } \\
\hline & & & X & $\mathrm{Y}$ & Z \\
\hline 1 & 16 & 0 & -5.971429 & -2.262865 & 0.924691 \\
\hline 2 & 16 & 0 & -7.771831 & 1.342039 & -0.972000 \\
\hline 3 & 16 & 0 & -2.614014 & -2.664652 & 1.982976 \\
\hline 4 & 16 & 0 & -1.766577 & 3.417575 & -2.242559 \\
\hline 5 & 16 & 0 & 1.717537 & 3.363599 & 2.101033 \\
\hline 6 & 16 & 0 & 2.661939 & -2.598176 & -2.335622 \\
\hline 7 & 16 & 0 & 7.788231 & 1.380300 & 1.016301 \\
\hline 8 & 16 & 0 & 6.014895 & -2.239431 & -0.887791 \\
\hline 9 & 7 & 0 & -4.783384 & -4.649499 & 2.134504 \\
\hline 10 & 7 & 0 & -0.163946 & 0.506679 & 2.110175 \\
\hline 11 & 7 & 0 & 4.397620 & 4.567913 & 2.210387 \\
\hline 12 & 7 & 0 & -4.449334 & 4.610417 & -2.130453 \\
\hline 13 & 7 & 0 & -2.109096 & 0.222295 & 1.035435 \\
\hline 14 & 7 & 0 & 0.119236 & 0.568477 & -2.217446 \\
\hline 15 & 7 & 0 & 1.961097 & 1.809048 & -1.898402 \\
\hline 16 & 7 & 0 & 4.839043 & -4.586482 & -2.181471 \\
\hline 17 & 6 & 0 & 2.487213 & 6.050191 & 2.607589 \\
\hline 18 & 6 & 0 & -0.619859 & 1.771760 & 1.753011 \\
\hline 19 & 6 & 0 & -8.491851 & -5.396679 & 2.049555 \\
\hline 20 & 6 & 0 & -9.197738 & -5.284288 & 3.265889 \\
\hline 21 & 6 & 0 & -10.367794 & -6.030110 & 3.445303 \\
\hline 22 & 1 & 0 & -10.906428 & -5.941801 & 4.386365 \\
\hline 23 & 6 & 0 & -10.859687 & -6.883780 & 2.454460 \\
\hline 24 & 6 & 0 & -10.141533 & -6.985372 & 1.259792 \\
\hline 25 & 1 & 0 & -10.501825 & -7.650520 & 0.477843 \\
\hline 26 & 6 & 0 & -8.967653 & -6.257672 & 1.037675 \\
\hline 27 & 6 & 0 & -7.224791 & -4.611222 & 1.839539 \\
\hline 28 & 6 & 0 & -7.326705 & -3.351367 & 1.268318 \\
\hline 29 & 6 & 0 & -8.556226 & -2.738612 & 0.849078 \\
\hline 30 & 1 & 0 & -9.504380 & -3.245151 & 0.977164 \\
\hline 31 & 6 & 0 & -8.413563 & -1.502950 & 0.287896 \\
\hline 32 & 1 & 0 & -9.241643 & -0.909901 & -0.084049 \\
\hline 33 & 6 & 0 & -7.063730 & -1.043768 & 0.226583 \\
\hline 34 & 6 & 0 & -8.706772 & -4.377155 & 4.371278 \\
\hline 35 & 1 & 0 & -9.360274 & -4.439037 & 5.245414 \\
\hline 36 & 1 & 0 & -8.671699 & -3.331112 & 4.048333 \\
\hline 37 & 1 & 0 & -7.692094 & -4.642148 & 4.686633 \\
\hline 38 & 6 & 0 & -12.142856 & -7.654372 & 2.657710 \\
\hline
\end{tabular}




\begin{tabular}{|c|c|c|c|c|c|}
\hline 39 & 1 & 0 & -12.319288 & -7.866416 & 3.716438 \\
\hline 40 & 1 & 0 & -12.128028 & -8.606607 & 2.118764 \\
\hline 41 & 1 & 0 & -13.008354 & -7.088103 & 2.291102 \\
\hline 42 & 6 & 0 & -8.225636 & -6.408336 & -0.270954 \\
\hline 43 & 1 & 0 & -7.202244 & -6.764998 & -0.112177 \\
\hline 44 & 1 & 0 & -8.146632 & -5.453758 & -0.801848 \\
\hline 45 & 1 & 0 & -8.734179 & -7.119511 & -0.927216 \\
\hline 46 & 6 & 0 & -5.989106 & -5.226627 & 2.226793 \\
\hline 47 & 6 & 0 & -5.889442 & -6.594007 & 2.748032 \\
\hline 48 & 1 & 0 & -6.720287 & -7.262894 & 2.922981 \\
\hline 49 & 6 & 0 & -4.569754 & -6.826788 & 2.948736 \\
\hline 50 & 1 & 0 & -4.099562 & -7.723381 & 3.327125 \\
\hline 51 & 6 & 0 & -3.874323 & -5.599512 & 2.564110 \\
\hline 52 & 6 & 0 & -6.631377 & 0.166995 & -0.288711 \\
\hline 53 & 6 & 0 & -6.466847 & 2.498936 & -1.322583 \\
\hline 54 & 6 & 0 & -5.212084 & 1.933116 & -0.919222 \\
\hline 55 & 1 & 0 & -4.299790 & 2.495580 & -1.061531 \\
\hline 56 & 6 & 0 & -5.300751 & 0.686237 & -0.360582 \\
\hline 57 & 1 & 0 & -4.442822 & 0.140545 & 0.022582 \\
\hline 58 & 6 & 0 & -2.499866 & -5.418147 & 2.625589 \\
\hline 59 & 6 & 0 & -1.814363 & -4.190817 & 2.332054 \\
\hline 60 & 6 & 0 & -1.080284 & -1.826103 & 1.895068 \\
\hline 61 & 6 & 0 & -0.016281 & -2.695945 & 2.092424 \\
\hline 62 & 1 & 0 & 1.023764 & -2.402732 & 2.026275 \\
\hline 63 & 6 & 0 & -0.428423 & -4.016429 & 2.337596 \\
\hline 64 & 1 & 0 & 0.255746 & -4.836550 & 2.515176 \\
\hline 65 & 6 & 0 & -6.761088 & 3.738938 & -1.862164 \\
\hline 66 & 6 & 0 & -5.781275 & 4.755194 & -2.166538 \\
\hline 67 & 6 & 0 & -3.924164 & 5.850212 & -2.441659 \\
\hline 68 & 6 & 0 & -4.993984 & 6.812247 & -2.678016 \\
\hline 69 & 1 & 0 & -4.860525 & 7.852833 & -2.938265 \\
\hline 70 & 6 & 0 & -6.155391 & 6.126654 & -2.527917 \\
\hline 71 & 1 & 0 & -7.165574 & 6.496186 & -2.632664 \\
\hline 72 & 6 & 0 & -1.647818 & -6.588792 & 3.032532 \\
\hline 73 & 6 & 0 & -1.197361 & -6.714801 & 4.364359 \\
\hline 74 & 6 & 0 & -0.422230 & -7.824847 & 4.714682 \\
\hline 75 & 1 & 0 & -0.084308 & -7.923305 & 5.743995 \\
\hline 76 & 6 & 0 & -0.078265 & -8.811180 & 3.786006 \\
\hline 77 & 6 & 0 & -0.540212 & -8.667613 & 2.475163 \\
\hline 78 & 1 & 0 & -0.296583 & -9.430965 & 1.739221 \\
\hline 79 & 6 & 0 & -1.318067 & -7.573819 & 2.078502 \\
\hline 80 & 6 & 0 & -1.102130 & -0.400152 & 1.657152 \\
\hline 81 & 6 & 0 & -1.821713 & 1.545454 & 1.093658 \\
\hline 82 & 1 & 0 & -2.457160 & 2.292056 & 0.640612 \\
\hline
\end{tabular}




\begin{tabular}{|c|c|c|c|c|c|}
\hline 83 & 6 & 0 & -8.203634 & 4.073439 & -2.129842 \\
\hline 84 & 6 & 0 & -8.997706 & 4.660988 & -1.120814 \\
\hline 85 & 6 & 0 & -10.333404 & 4.966078 & -1.400308 \\
\hline 86 & 1 & 0 & -10.940250 & 5.423059 & -0.621537 \\
\hline 87 & 6 & 0 & -10.907316 & 4.703283 & -2.647341 \\
\hline 88 & 6 & 0 & -10.101925 & 4.127852 & -3.633262 \\
\hline 89 & 1 & 0 & -10.525703 & 3.923206 & -4.614014 \\
\hline 90 & 6 & 0 & -8.760391 & 3.808836 & -3.399314 \\
\hline 91 & 6 & 0 & -2.566236 & 6.124192 & -2.538991 \\
\hline 92 & 6 & 0 & -1.519733 & 5.155861 & -2.417861 \\
\hline 93 & 6 & 0 & -0.037513 & 3.114569 & -2.253058 \\
\hline 94 & 6 & 0 & 0.670829 & 4.306311 & -2.364276 \\
\hline 95 & 1 & 0 & 1.752418 & 4.320787 & -2.373242 \\
\hline 96 & 6 & 0 & -0.150412 & 5.432876 & -2.460768 \\
\hline 97 & 1 & 0 & 0.222835 & 6.443519 & -2.567329 \\
\hline 98 & 6 & 0 & -1.557186 & -5.688573 & 5.414451 \\
\hline 99 & 1 & 0 & -1.198473 & -5.997769 & 6.399558 \\
\hline 100 & 1 & 0 & -2.640571 & -5.543332 & 5.475997 \\
\hline 101 & 1 & 0 & -1.120004 & -4.710329 & 5.187534 \\
\hline 102 & 6 & 0 & 0.786309 & -9.984245 & 4.183458 \\
\hline 103 & 1 & 0 & 0.623458 & -10.265652 & 5.228223 \\
\hline 104 & 1 & 0 & 1.851924 & -9.746711 & 4.074074 \\
\hline 105 & 1 & 0 & 0.583727 & -10.860606 & 3.560626 \\
\hline 106 & 6 & 0 & -1.806465 & -7.475630 & 0.651015 \\
\hline 107 & 1 & 0 & -2.880681 & -7.680493 & 0.582486 \\
\hline 108 & 1 & 0 & -1.284776 & -8.187151 & 0.007304 \\
\hline 109 & 1 & 0 & -1.649891 & -6.473293 & 0.241162 \\
\hline 110 & 6 & 0 & -0.008475 & 3.047724 & 2.042489 \\
\hline 111 & 6 & 0 & -0.728632 & 4.221691 & 2.217646 \\
\hline 112 & 1 & 0 & -1.811597 & 4.246815 & 2.228616 \\
\hline 113 & 6 & 0 & 0.081969 & 5.348823 & 2.410258 \\
\hline 114 & 1 & 0 & -0.302796 & 6.345961 & 2.581628 \\
\hline 115 & 6 & 0 & 1.451623 & 5.081142 & 2.390910 \\
\hline 116 & 6 & 0 & 0.979702 & 0.220984 & 2.968407 \\
\hline 117 & 1 & 0 & 1.153873 & 1.071169 & 3.627730 \\
\hline 118 & 1 & 0 & 1.886132 & 0.040185 & 2.382368 \\
\hline 119 & 1 & 0 & 0.761371 & -0.659894 & 3.572407 \\
\hline 120 & 6 & 0 & -8.423379 & 4.974431 & 0.241868 \\
\hline 121 & 1 & 0 & -8.057296 & 4.070540 & 0.739917 \\
\hline 122 & 1 & 0 & -9.179032 & 5.431848 & 0.885938 \\
\hline 123 & 1 & 0 & -7.576168 & 5.665114 & 0.170686 \\
\hline 124 & 6 & 0 & -12.362349 & 5.010306 & -2.911915 \\
\hline 125 & 1 & 0 & -12.693196 & 5.892697 & -2.355587 \\
\hline 126 & 1 & 0 & -13.005253 & 4.175103 & -2.606695 \\
\hline
\end{tabular}




\begin{tabular}{|c|c|c|c|c|c|}
\hline 127 & 1 & 0 & -12.547049 & 5.190330 & -3.975104 \\
\hline 128 & 6 & 0 & -7.928646 & 3.195807 & -4.502671 \\
\hline 129 & 1 & 0 & -7.066840 & 3.824247 & -4.751855 \\
\hline 130 & 1 & 0 & -8.523425 & 3.062351 & -5.410110 \\
\hline 131 & 1 & 0 & -7.533836 & 2.217146 & -4.210304 \\
\hline 132 & 6 & 0 & -2.135775 & 7.544091 & -2.790464 \\
\hline 133 & 6 & 0 & -1.924416 & 7.996817 & -4.109858 \\
\hline 134 & 6 & 0 & -1.521845 & 9.320504 & -4.315062 \\
\hline 135 & 1 & 0 & -1.360735 & 9.666362 & -5.333742 \\
\hline 136 & 6 & 0 & -1.318955 & 10.205797 & -3.252895 \\
\hline 137 & 6 & 0 & -1.525867 & 9.731996 & -1.954706 \\
\hline 138 & 1 & 0 & -1.368022 & 10.401890 & -1.112196 \\
\hline 139 & 6 & 0 & -1.933950 & 8.417844 & -1.701489 \\
\hline 140 & 6 & 0 & 0.639155 & 1.844451 & -2.117331 \\
\hline 141 & 6 & 0 & 1.181856 & -0.314099 & -2.044403 \\
\hline 142 & 6 & 0 & 2.295087 & 0.507196 & -1.859383 \\
\hline 143 & 1 & 0 & 3.315189 & 0.187758 & -1.697770 \\
\hline 144 & 6 & 0 & 3.847862 & 5.791215 & 2.548347 \\
\hline 145 & 6 & 0 & 4.900472 & 6.759953 & 2.836679 \\
\hline 146 & 1 & 0 & 4.747410 & 7.789938 & 3.126583 \\
\hline 147 & 6 & 0 & 6.073380 & 6.098105 & 2.678863 \\
\hline 148 & 1 & 0 & 7.076266 & 6.479616 & 2.807797 \\
\hline 149 & 6 & 0 & 5.725114 & 4.731369 & 2.270262 \\
\hline 150 & 6 & 0 & -2.129382 & 7.079972 & -5.294114 \\
\hline 151 & 1 & 0 & -1.926453 & 7.603755 & -6.231723 \\
\hline 152 & 1 & 0 & -3.155453 & 6.699464 & -5.332371 \\
\hline 153 & 1 & 0 & -1.469615 & 6.207245 & -5.244378 \\
\hline 154 & 6 & 0 & -0.913703 & 11.639242 & -3.502742 \\
\hline 155 & 1 & 0 & -0.335105 & 12.043682 & -2.666833 \\
\hline 156 & 1 & 0 & -1.793015 & 12.283050 & -3.630585 \\
\hline 157 & 1 & 0 & -0.309702 & 11.732598 & -4.410254 \\
\hline 158 & 6 & 0 & -2.154479 & 7.961405 & -0.276767 \\
\hline 159 & 1 & 0 & -1.824076 & 8.725361 & 0.431880 \\
\hline 160 & 1 & 0 & -1.607328 & 7.038555 & -0.058986 \\
\hline 161 & 1 & 0 & -3.213295 & 7.755806 & -0.084263 \\
\hline 162 & 6 & 0 & -1.249116 & 0.195664 & -2.561225 \\
\hline 163 & 1 & 0 & -1.842936 & 0.005236 & -1.661905 \\
\hline 164 & 1 & 0 & -1.226044 & -0.699675 & -3.183168 \\
\hline 165 & 1 & 0 & -1.709763 & 0.998300 & -3.136032 \\
\hline 166 & 6 & 0 & 1.142085 & -1.752726 & -2.089564 \\
\hline 167 & 6 & 0 & 0.095189 & -2.658258 & -1.940338 \\
\hline 168 & 1 & 0 & -0.923999 & -2.373627 & -1.715810 \\
\hline 169 & 6 & 0 & 0.503313 & -3.997632 & -2.059493 \\
\hline 170 & 1 & 0 & -0.173498 & -4.839737 & -1.981910 \\
\hline
\end{tabular}




\begin{tabular}{|c|c|c|c|c|c|}
\hline 171 & 6 & 0 & 1.871349 & -4.166864 & -2.276182 \\
\hline 172 & 6 & 0 & 2.032753 & 7.451953 & 2.912291 \\
\hline 173 & 6 & 0 & 1.773158 & 7.836987 & 4.245265 \\
\hline 174 & 6 & 0 & 1.344267 & 9.142522 & 4.501861 \\
\hline 175 & 1 & 0 & 1.145195 & 9.435211 & 5.530583 \\
\hline 176 & 6 & 0 & 1.162807 & 10.076930 & 3.477607 \\
\hline 177 & 6 & 0 & 1.420258 & 9.671241 & 2.166226 \\
\hline 178 & 1 & 0 & 1.282609 & 10.380707 & 1.353211 \\
\hline 179 & 6 & 0 & 1.854651 & 8.375573 & 1.861811 \\
\hline 180 & 6 & 0 & 6.726706 & 3.741685 & 1.946963 \\
\hline 181 & 6 & 0 & 6.455867 & 2.492737 & 1.416466 \\
\hline 182 & 6 & 0 & 5.205155 & 1.879390 & 1.074544 \\
\hline 183 & 1 & 0 & 4.277175 & 2.409988 & 1.234364 \\
\hline 184 & 6 & 0 & 5.323467 & 0.628146 & 0.534688 \\
\hline 185 & 1 & 0 & 4.476081 & 0.027963 & 0.223389 \\
\hline 186 & 6 & 0 & 6.665326 & 0.159921 & 0.385960 \\
\hline 187 & 6 & 0 & 2.546505 & -5.409359 & -2.511101 \\
\hline 188 & 6 & 0 & 3.927414 & -5.567008 & -2.531752 \\
\hline 189 & 6 & 0 & 4.625483 & -6.787388 & -2.924720 \\
\hline 190 & 1 & 0 & 4.155239 & -7.702565 & -3.255850 \\
\hline 191 & 6 & 0 & 5.950871 & -6.519184 & -2.814660 \\
\hline 192 & 1 & 0 & 6.784541 & -7.174669 & -3.023727 \\
\hline 193 & 6 & 0 & 6.049306 & -5.141545 & -2.326643 \\
\hline 194 & 6 & 0 & 1.958071 & 6.866191 & 5.389243 \\
\hline 195 & 1 & 0 & 2.997084 & 6.527701 & 5.461778 \\
\hline 196 & 1 & 0 & 1.341954 & 5.970467 & 5.258547 \\
\hline 197 & 1 & 0 & 1.685314 & 7.329129 & 6.341082 \\
\hline 198 & 6 & 0 & 0.723688 & 11.488671 & 3.787250 \\
\hline 199 & 1 & 0 & -0.037205 & 11.508048 & 4.574016 \\
\hline 200 & 1 & 0 & 0.309828 & 11.982423 & 2.903429 \\
\hline 201 & 1 & 0 & 1.565820 & 12.097783 & 4.138542 \\
\hline 202 & 6 & 0 & 2.130857 & 7.994855 & 0.424640 \\
\hline 203 & 1 & 0 & 3.199354 & 7.819231 & 0.257948 \\
\hline 204 & 1 & 0 & 1.808382 & 8.786974 & -0.256187 \\
\hline 205 & 1 & 0 & 1.611665 & 7.073418 & 0.142796 \\
\hline 206 & 6 & 0 & 8.165242 & 4.116585 & 2.180544 \\
\hline 207 & 6 & 0 & 8.912701 & 4.740339 & 1.158021 \\
\hline 208 & 6 & 0 & 10.248647 & 5.074577 & 1.401894 \\
\hline 209 & 1 & 0 & 10.821667 & 5.552440 & 0.610204 \\
\hline 210 & 6 & 0 & 10.865351 & 4.811719 & 2.628047 \\
\hline 211 & 6 & 0 & 10.107334 & 4.193744 & 3.626070 \\
\hline 212 & 1 & 0 & 10.568447 & 3.976857 & 4.587190 \\
\hline 213 & 6 & 0 & 8.768060 & 3.843902 & 3.427375 \\
\hline 214 & 6 & 0 & 7.108196 & -1.018826 & -0.189378 \\
\hline
\end{tabular}




\begin{tabular}{|c|c|c|c|c|c|}
\hline 215 & 6 & 0 & 7.383293 & -3.262506 & -1.369695 \\
\hline 216 & 6 & 0 & 8.613558 & -2.626530 & -0.988451 \\
\hline 217 & 1 & 0 & 9.568393 & -3.091513 & -1.198764 \\
\hline 218 & 6 & 0 & 8.465657 & -1.428387 & -0.352034 \\
\hline 219 & 1 & 0 & 9.295087 & -0.827222 & 0.003562 \\
\hline 220 & 6 & 0 & 1.691095 & -6.618439 & -2.763401 \\
\hline 221 & 6 & 0 & 1.084487 & -6.806339 & -4.025866 \\
\hline 222 & 6 & 0 & 0.330117 & -7.961309 & -4.249563 \\
\hline 223 & 1 & 0 & -0.124137 & -8.107783 & -5.227095 \\
\hline 224 & 6 & 0 & 0.153413 & -8.934541 & -3.261330 \\
\hline 225 & 6 & 0 & 0.762269 & -8.727450 & -2.021626 \\
\hline 226 & 1 & 0 & 0.648113 & -9.477643 & -1.242112 \\
\hline 227 & 6 & 0 & 1.526893 & -7.586372 & -1.750125 \\
\hline 228 & 6 & 0 & 7.288025 & -4.497279 & -1.990684 \\
\hline 229 & 6 & 0 & 8.562531 & -5.231552 & -2.310603 \\
\hline 230 & 6 & 0 & 9.148945 & -6.089344 & -1.355990 \\
\hline 231 & 6 & 0 & 10.328178 & -6.767042 & -1.684163 \\
\hline 232 & 1 & 0 & 10.777272 & -7.425626 & -0.943836 \\
\hline 233 & 6 & 0 & 10.942342 & -6.621333 & -2.930851 \\
\hline 234 & 6 & 0 & 10.346599 & -5.764146 & -3.860120 \\
\hline 235 & 1 & 0 & 10.810810 & -5.631230 & -4.834980 \\
\hline 236 & 6 & 0 & 9.167251 & -5.068167 & -3.575190 \\
\hline 237 & 6 & 0 & 8.291002 & 5.053648 & -0.183682 \\
\hline 238 & 1 & 0 & 7.431241 & 5.724332 & -0.079591 \\
\hline 239 & 1 & 0 & 7.929228 & 4.147214 & -0.680078 \\
\hline 240 & 1 & 0 & 9.016474 & 5.534335 & -0.845216 \\
\hline 241 & 6 & 0 & 12.301026 & 5.210133 & 2.877131 \\
\hline 242 & 1 & 0 & 12.366041 & 6.228333 & 3.281155 \\
\hline 243 & 1 & 0 & 12.887972 & 5.189948 & 1.953991 \\
\hline 244 & 1 & 0 & 12.781813 & 4.543810 & 3.599611 \\
\hline 245 & 6 & 0 & 7.988815 & 3.183636 & 4.541692 \\
\hline 246 & 1 & 0 & 8.611712 & 3.059272 & 5.431414 \\
\hline 247 & 1 & 0 & 7.623010 & 2.195266 & 4.244162 \\
\hline 248 & 1 & 0 & 7.111271 & 3.776017 & 4.822439 \\
\hline 249 & 6 & 0 & 1.260843 & -5.802076 & -5.142291 \\
\hline 250 & 1 & 0 & 0.751866 & -4.858266 & -4.919369 \\
\hline 251 & 1 & 0 & 0.854431 & -6.189481 & -6.080092 \\
\hline 252 & 1 & 0 & 2.316838 & -5.561948 & -5.301607 \\
\hline 253 & 6 & 0 & -0.689423 & -10.160251 & -3.522808 \\
\hline 254 & 1 & 0 & -0.424449 & -10.980114 & -2.848893 \\
\hline 255 & 1 & 0 & -0.571295 & -10.514868 & -4.551449 \\
\hline 256 & 1 & 0 & -1.755489 & -9.947123 & -3.375294 \\
\hline 257 & 6 & 0 & 2.166901 & -7.422848 & -0.389213 \\
\hline 258 & 1 & 0 & 3.238630 & -7.648277 & -0.422726 \\
\hline
\end{tabular}




\begin{tabular}{rrrrrr}
259 & 1 & 0 & 1.704162 & -8.090608 & 0.341980 \\
260 & 1 & 0 & 2.071268 & -6.398142 & -0.019088 \\
261 & 6 & 0 & 8.525727 & -6.282723 & 0.007983 \\
262 & 1 & 0 & 9.087166 & -7.016749 & 0.592051 \\
263 & 1 & 0 & 7.490334 & -6.630718 & -0.070049 \\
264 & 1 & 0 & 8.501040 & -5.346745 & 0.576424 \\
265 & 6 & 0 & 12.197822 & -7.388207 & -3.273779 \\
266 & 1 & 0 & 12.792368 & -7.602710 & -2.380746 \\
267 & 1 & 0 & 12.827522 & -6.832783 & -3.975386 \\
268 & 1 & 0 & 11.959158 & -8.350419 & -3.744190 \\
269 & 6 & 0 & 8.563615 & -4.152000 & -4.615268 \\
270 & 1 & 0 & 8.497650 & -3.120596 & -4.253141 \\
271 & 1 & 0 & 7.545075 & -4.457958 & -4.876885 \\
272 & 1 & 0 & 9.162599 & -4.152353 & -5.529731 \\
\hline
\end{tabular}

